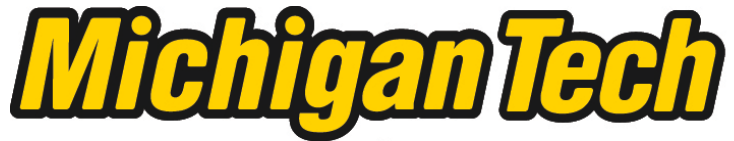 \\ Michigan Technological University Create the Future Digital Commons @ Michigan Tech
}

2013

\section{MAGNETO-PHOTONIC CRYSTALS FOR OPTICAL SENSING APPLICATIONS}

Neluka Dissanayake

Michigan Technological University

Follow this and additional works at: https://digitalcommons.mtu.edu/etds

Part of the Engineering Commons, and the Optics Commons Copyright 2013 Neluka Dissanayake

\section{Recommended Citation}

Dissanayake, Neluka, "MAGNETO-PHOTONIC CRYSTALS FOR OPTICAL SENSING APPLICATIONS", Dissertation, Michigan Technological University, 2013.

https://doi.org/10.37099/mtu.dc.etds/628

Follow this and additional works at: https://digitalcommons.mtu.edu/etds

Part of the Engineering Commons, and the Optics Commons 
MAGNETO-PHOTONIC CRYSTALS FOR OPTICAL SENSING APPLICATIONS

\author{
By
}

Neluka Dissanayake

\begin{abstract}
A DISSERTATION
Submitted in partial fulfillment of the requirements for the degree of DOCTOR OF PHILOSOPHY
\end{abstract}

In Engineering Physics

MICHIGAN TECHNOLOGICAL UNIVERSITY

2013 
This dissertation has been approved in partial fulfillment of the requirements for the Degree of DOCTOR OF PHILOSOPHY in Engineering Physics.

Department of Physics

Dissertation Advisor: Dr. Miguel Levy

Committee Member: Dr. Brian Fick

Committee Member: Dr. Ranjit Pati

Committee Member: Dr. Christopher T. Middlebrook

Department Chair: Dr. Ravindra Pandey 


\section{TABLE OF CONTENTS}

List of Figures.................................................................................................................................... vii

List of Tables .......................................................................................................... xii

Acknowledgements .................................................................................................................... xiii

Abbreviations .............................................................................................................................

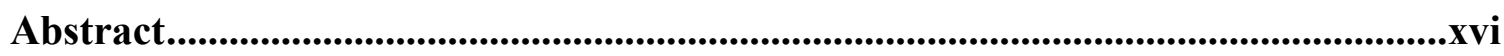

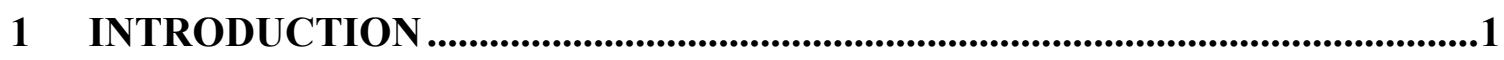

1.1 Photonic crystals for optical sensing ..............................................................

1.2 Magneto-photonic crystal optical sensors ..........................................................

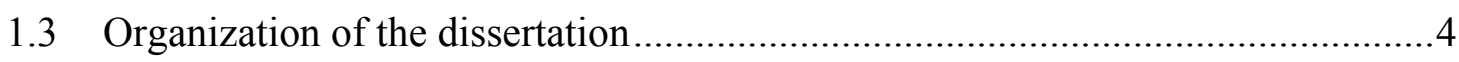

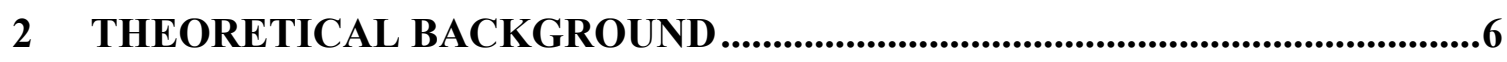

2.1 Planar dielectric waveguides ..................................................................6

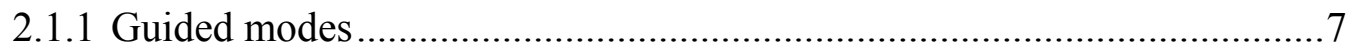

2.1.2 Effective or mode index ……...............................................................

2.1.3 Waveguide Theory …………………………...................................

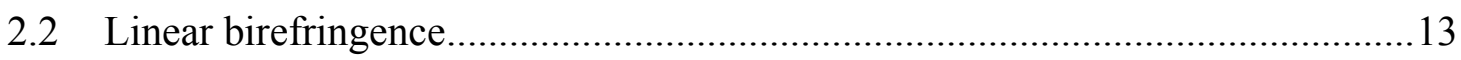

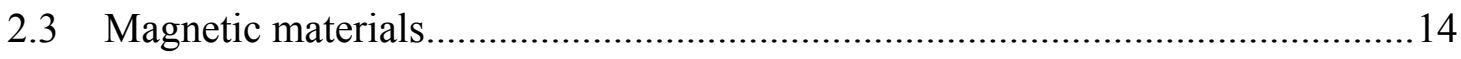

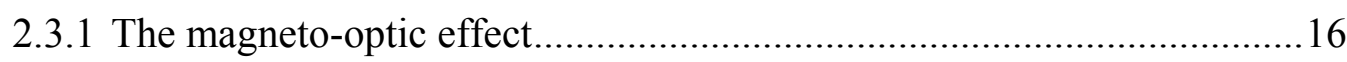

2.3.1.1 Faraday effect.............................................................................16

2.4 Elliptical birefringence and polarization rotation ............................................19

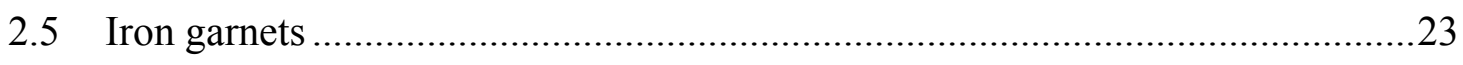

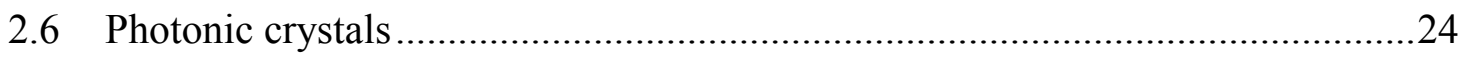

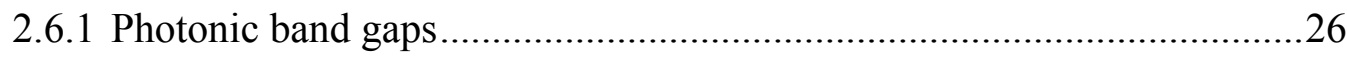

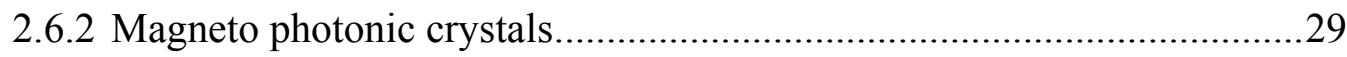

2.6.3 One dimensional Bragg waveguides .........................................................30

2.7 Wave propagation in birefringent magneto-optic media .......................................32 
2.8 Gyrotropic bandgaps in elliptically birefringent media......................................34

2.9 Conducting polymers for chemical sensing .......................................................36

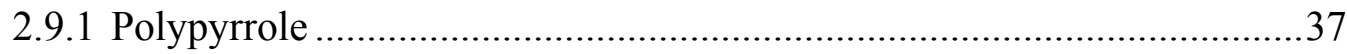

2.9.2 Sensing mechanism ................................................................................ 38

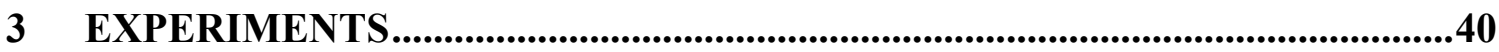

3.1 Iron garnet thin film fabrication .................................................................... 40

3.1.1 Liquid phase epitaxy technique ............................................................. 40

3.1.2 Magnetron sputtering ............................................................................ 41

3.2 Thin film characterization...........................................................................

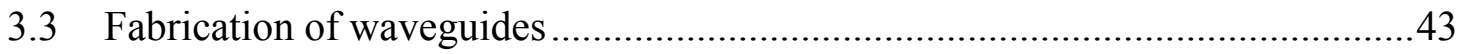

3.3.1 Waveguide dimension optimization .......................................................43

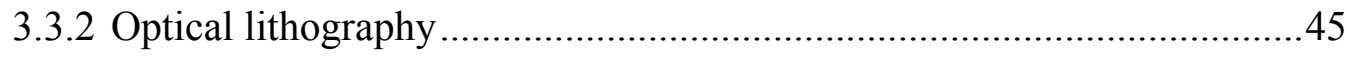

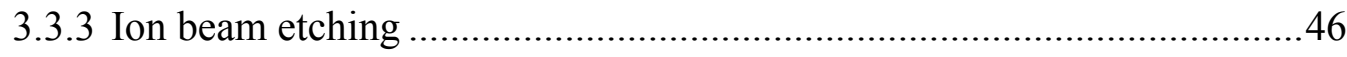

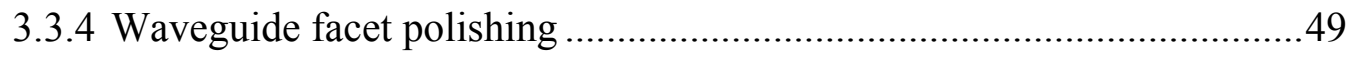

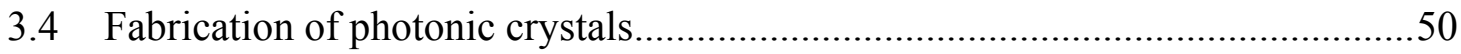

3.4.1 Focused ion beam (FIB) milling …………………............................50

3.4.2 Grating depth characterization ...............................................................5

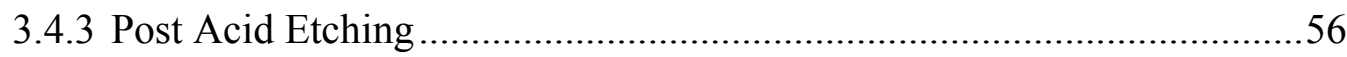

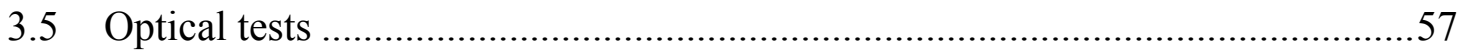

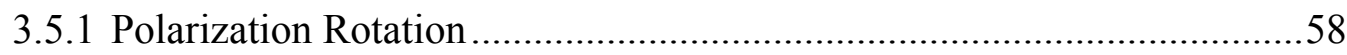

3.5.2 Rotating polarizer technique. ...............................................................58

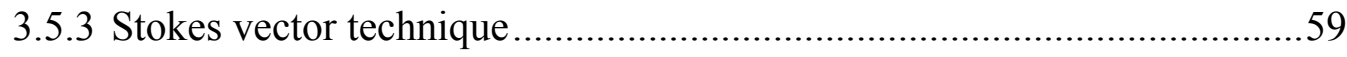

3.6 Synthesis and characterization of Polypyrrole .....................................................61

3.6.1 Chemical oxidation of Polypyrrole …………………..............................62

3.6.2 Ellipsometric characterization of Polypyrrole...........................................64

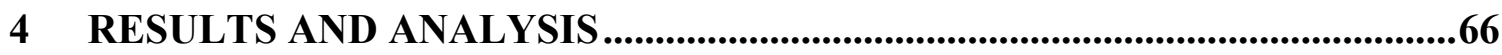

4.1 Ridge waveguides and mode propagation …………….....................................66 


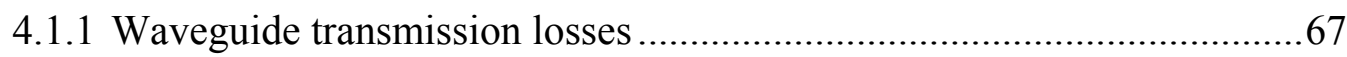

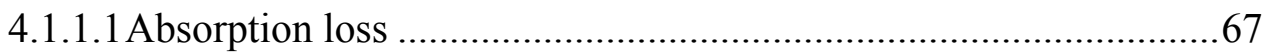

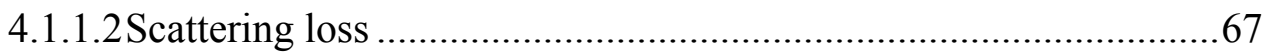

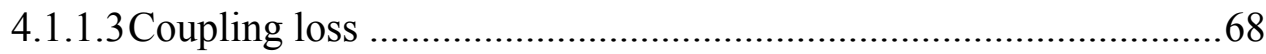

4.2 Magneto-optic Bragg gratings ...........................................................................68

4.2.1 TE and TM band gaps ......................................................................

4.3 Polarization rotation in magneto-optic Bragg gratings........................................

4.3.1 Experiment and theoretical evidence .....................................................74

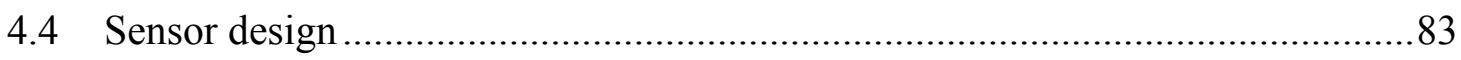

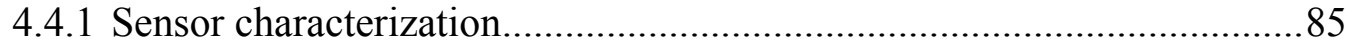

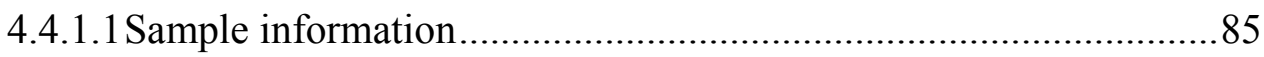

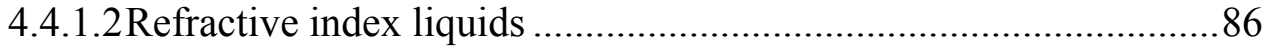

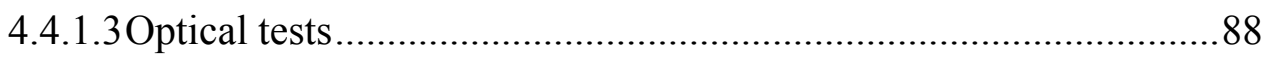

4.4.2 Theoretical simulation ...........................................................................92

4.4.3 Photonic crystal and plain waveguide polarization rotation comparison. 94

4.4.4 Sensor sensitivity ...................................................................................97

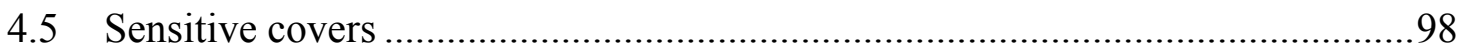

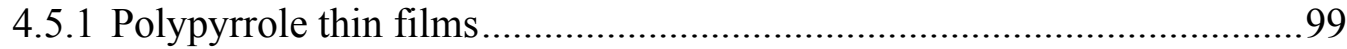

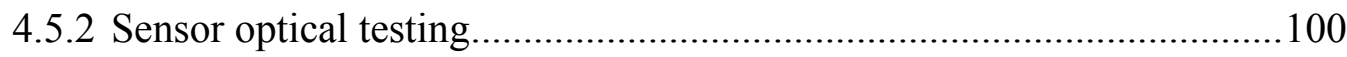

4.5.3 Tests with aqueous ammonia and methanol.............................................103

4.5.4 Ellipsometric characterization of Polypyrrole.........................................106

5 SUMMARY AND FUTURE WORK ...................................................................108

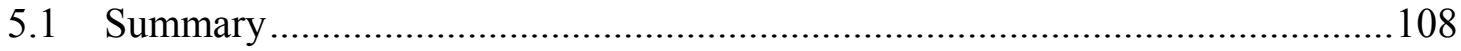

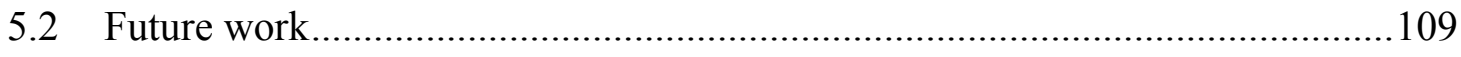




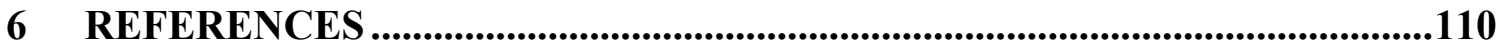

PUBLICATIONS/PATENT APPLICATIONS...........................................................119

Appendix A

Appendix B .......................................................................................................................121 


\section{List of Figures}

Figure 1.1 (a),(b) Scanning electron microscope view of a photonic crystal microcavity integrated with two ridge waveguides, normalized transmission spectra of the photonic crystal microcavity [shown in (a)] with five different ambient refractive indices ranging from $n=1.446$ to $n=1.454$. (c),(d) Photonic crystal waveguide (PC-WG) on SOI wafer, measured transmittance spectra from the fabricated $\mathrm{PC}-\mathrm{WG}$ for four different cover solutions [5,8]. (Reprinted from refs. 5 and 8, permission details in Appendix B) .................2

Figure 1.2 Magneto-photonic crystal sensor with a receptor layer applied on the grating

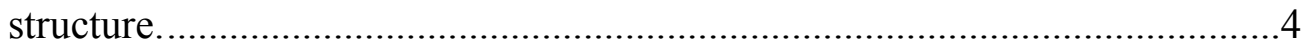

Figure 2.1 Basic slab waveguide structure ..........................................................6

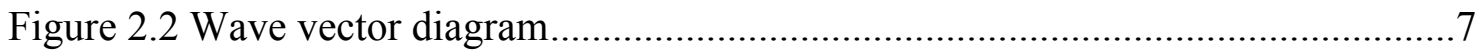

Figure 2.3 First four waveguide modes supported by a $2.71 \mu \mathrm{m}$ thick ridge waveguide. The effective indices of the modes vary between the substrate index 1.95 and film index 2.31 .

Figure 2.4 Calculated TE and TM waveguide modes for a garnet film with refractive index 2.31 at a wavelength of $1543 \mathrm{~nm}$.

Figure 2.5 Magnetic dipole arrangements in magnetic materials ............................. 15

Figure 2.6 (a) Geometry of the Faraday effect. (b) Non-reciprocal character of the Faraday effect. .16

Figure 2.7 Faraday rotation in media with linear birefringence.

Figure 2.8 Linearly polarized light converted in to elliptically polarized light and rotated through an angle $\theta$ when propagated through birefringent magnetooptic media.

Figure 2.9 Lattice structure of yttrium iron garnet . (Courtesy: Zhuoyuan $\mathrm{Wu} \mathrm{PhD}$ disertation, permission details in appendix B) ......................................23

Figure 2.10 Periodic layer arrangement of simple 1-D, 2-D and 3-D photonic crystal structures

Figure 2.11 Left: Dispersion diagram of a homogeneous one-dimensional medium. Right: Band diagram of a one dimensional material system with a dielectric variation of period $a$. A gap is opened at the Brillouin zone boundary at $\mathrm{k}= \pm \boldsymbol{\pi} \boldsymbol{a}$. Inset: The dielectric periodicity in the system breaks plane waves of a uniform medium into $\cos (\pi \mathrm{x} / \mathrm{a})$ and $\sin (\pi \mathrm{x} / \mathrm{a})$ standing waves [38]......28 
Figure 2.12 Schematic depiction of the Bragg grating on magneto-optic ridge waveguide with SEM micrograph of the grating region fabricated by FIB milling 30

Figure 2.13 Contra directional coupling of waveguide modes by a grating. 31

Figure 2.14 Schematic diagram of a one-dimensional birefringent magneto-photonic crystal with period of $\Lambda$. The structure extends indefinitely in the $x$ and $y$ directions. A unit cell is given by the region between $z_{n-1}$ and $z_{n+1}$. [13] ......34

Figure 2.15(a) Pyrrole monomer, (b) neutral Polypyrrole, (c) oxidized Polypyrrole.....38

Figure 3.1 Schematic representation of the prism coupler set up.... 42

Figure 3.2 A screen shot of the prism coupler results for a $5 \mu \mathrm{m}$ thick film showing several guided modes for input TE polarization. .42

Figure 3.3 Ridge waveguide $\mathrm{x}-\mathrm{y}$ cross section .44

Figure 3.4 Transmitted power of a ridge waveguide designed on a film with thickness $2,71 \mu \mathrm{m}$ and refractive index 2.31. Transmitted power along the waveguide varies with the ridge height of the waveguide. 44

Figure $3.56 \mu \mathrm{m}$ wide waveguide patterns transferred to iron garnet films by UV optical lithography. 46

Figure 3.6 Etch rate characterization for Ar ion plasma etching for magnetic garnet thin

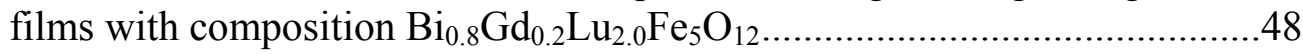

Figure 3.7 An interferrometric image of the waveguides after dry etching...................48

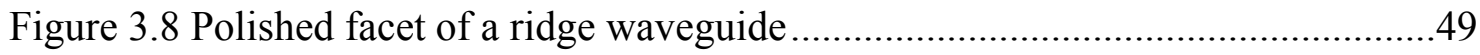

Figure 3.9 Schematic of focused ion beam system.............................................52

Figure 3.10 SEM micrograph of a misaligned FIB pattern due to charging effects .......53

Figure 3.11 SEM micrograph of a one-dimensional photonic crystal fabricated on $\mathrm{Bi}_{0.8} \mathrm{Gd}_{0.2} \mathrm{Lu}_{2.0} \mathrm{Fe}_{5} \mathrm{O}_{12}$ film .54

Figure 3.12 SEM micrograph of a one dimensional grating fabricated with underoptimized beam conditions. .54

Figure 3.13 FIB milling depth vs line dose for MI-100 beam for magnetic garnet

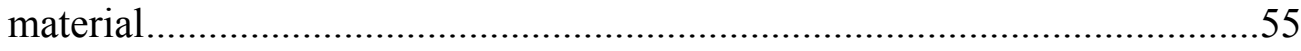

Figure 3.14 SEM micrograph of a grating cross section fabricated on

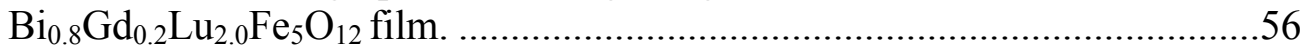

Figure 3.15 Transmittance spectrum of PC structure for pre and post acid etching.......57

Figure 3.16 Schematic of the optical measurement set-up .... .58 
Figure 3.17 In the rotating polarizer technique light intensity vs analyzer rotation angle. Left: for TE input, Right: Output polarization ellipse is rotated by angle $\theta^{\circ}$ with respect to the input TE light.

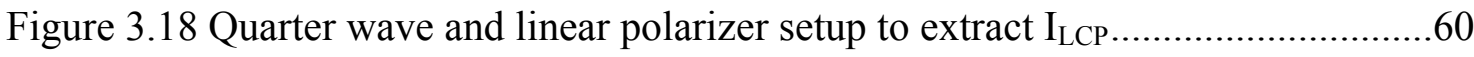

Figure 3.19 Quarter wave and linear polarizer setup to extract $I_{R C P}$ 61

Figure 3.20 The oxidant solution was applied on the PC with a fiber attached to a micromanipulator. This is performed under an optical microscope in order to apply the oxidant solution only on the $200 \mu \mathrm{m}$ long PC region.

Figure 3.21 Before and after deposition of PPy layer on the photonic crystal. The thickness of the PPy layer is controlled by the oxidant solution concentration and the Pyrrole vapor exposure time. .64

Figure 3.22 Schematic of polarized light and sample interaction in ellipsometer setup 65

Figure 4.1 Computed waveguide mode profiles of a ridge waveguide. .66

Figure 4.2 The transmittance spectrum of a one-dimensional photonic crystal with period $337 \mathrm{~nm}$ fabricated on a $2.71 \mu \mathrm{m}$-thick $\mathrm{Bi}_{0.8} \mathrm{Gd}_{0.2} \mathrm{Lu}_{2.0} \mathrm{Fe}_{5} \mathrm{O}_{12}$ film.

Figure 4.3 Stop bands corresponding to transverse electric and transverse magnetic input polarization states. .70

Figure 4.4 Polarization rotation measured for longitudinal magnetization (red) and for transverse magnetization (blue) showing large rotation for longitudinal magnetization and negligible rotation for a transverse field.

Figure 4.5 Polarization rotation measured for fundamental, first and second backscattered-order band gaps for longitudinal forward magnetization (red) and longitudinal backward magnetization (blue). These data show that the near band-edge polarization rotation increases with back-scattered mode order, and that the rotation is magneto-optic in character. .73

Figure 4.6 SEM micrograph of one-dimensional waveguide Bragg-filter without phase shift step. .75

Figure 4.7 SEM micrograph of one-dimensional waveguide Bragg-filter with resonant cavity .75

Figure 4.8 The figure plots the measured transmittance and polarization response [right panel], and calculated band structure and polarization rotation [left panel] of a one-dimensional photonic crystal with phase shift step of $3.5 \Lambda$ patterned on a $2.86 \mu$ m-thick $(\mathrm{Bi}, \mathrm{Lu})_{2.8} \mathrm{Fe}_{4.7} \mathrm{O}_{12.1}$ film (sample set A). Separate curves for the calculated semi-major axis orientation correspond to different Bloch states. The red and blue data points on the right panel describe the orientation of the semi-major axis of the polarization ellipse for opposite magnetizations collinear with the ridge waveguide axis. .78 
Figure 4.9 Measured transmittance and polarization response [right panel], and calculated band structure and polarization rotation [left panel] of a onedimensional Bragg filter without phase shift step patterned on a $2.7 \mu \mathrm{m}$-thick $\mathrm{Bi}_{0.8} \mathrm{Gd}_{0.2} \mathrm{Lu}_{2.0} \mathrm{Fe}_{5} \mathrm{O}_{12}$ film (sample set $\mathrm{B}$ ). Separate curves for the calculated semi-major axis orientation correspond to different Bloch states. The red and blue data points on the right describe the orientation of the semi-major axis of the output polarization ellipse for opposite magnetizations collinear with the ridge waveguide axis.

Figure 4.10 Measured transmittance and polarization response for a one-dimensional photonic crystal with a single phase shift step patterned on a $(\mathrm{Bi}, \mathrm{Lu}, \mathrm{Nd})_{3}(\mathrm{Fe}, \mathrm{Ga}, \mathrm{Al})_{5} \mathrm{O}_{12}$ film of thickness $1.8 \mu \mathrm{m}$. Black triangles and solid circles plot the orientation of the semi-major axis of the output polarization ellipse relative to the linear input polarization for opposite magnetization directions collinear with the ridge waveguide axis.

Figure 4.11 Measured ellipticity in the output polarization for Bragg filters patterned on ( $\mathrm{Bi}, \mathrm{Lu})_{2.8} \mathrm{Fe}_{4.7} \mathrm{O}_{12.1}$ film (sample set $\mathrm{A}$ ), $\mathrm{Bi}_{0.8} \mathrm{Gd}_{0.2} \mathrm{Lu}_{2.0} \mathrm{Fe}_{5} \mathrm{O}_{12}$ film (sample set $\mathrm{B}$ ) and $(\mathrm{Bi}, \mathrm{Lu}, \mathrm{Nd})_{3}(\mathrm{Fe}, \mathrm{Ga}, \mathrm{Al})_{5} \mathrm{O}_{12}$ film (samples set $\mathrm{C}$ ). Ellipticity is defined as the ratio of the semi-minor to semi-major axes of the polarization ellipse in the optical electric field amplitude. The horizontal double-tipped arrows indicate the locations of the stopbands in each case.

Figure 4.12 Angular dependence on index of the semi-major axis of the output polarization ellipse in the overlap pass band formed near the gyrotropic

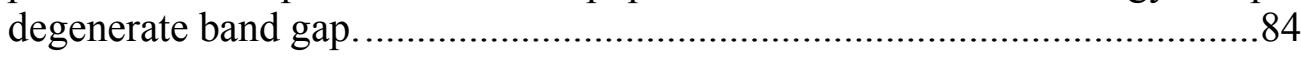

Figure 4.13 Initial sensor testing results for air cover and vacuum grease cover..........84

Figure 4.14 schematic representation of magneto-photonic crystal sensor with

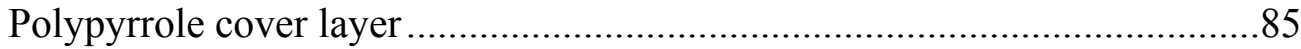

Figure 4.15Prism coupler measurement set up for bulk materials.............................87

Figure 4.16 Schematic picture of hotonic crystal cover modified with refractive index liquid applied over the PC.... 88

Figure 4.17 Measured transmittance and polarization rotation for varying cover index of the waveguide photonic crystal for sample 1 .90

Figure 4.18 Measured transmittance and polarization rotation for varying cover index of the waveguide photonic crystal for sample 2 . .90

Figure 4.19 Measured transmittance and polarization rotation for varying cover index of the waveguide photonic crystal for sample 3 91

Figure 4.20Average polarization rotation measured for samples 1, 2 and 3. .92 
Figure 4.21 Calculated band structure for a bilayer stack with period $\Lambda$ and computed orientation of the semi-major axis of one of the Bloch modes versus cover index.

Figure 4.22 Bloch mode polarization averaged over the band width rotation for increasing cover index .94

Figure 4.23 Transmittance and polarization rotation measured for a plain waveguide..95

Figure 4.24 Average polarization rotation versus cover index for a waveguide photonic crystal and for a plain waveguide 96

Figure 4.25 Polypyrrole deposited on a photonic crystal sample 100

Figure 4.26 Polarization rotation of a test PC measured for air and PPy cover. Transmittance is measured for air cover. 101

Figure 4.27 (a) Transmittance intensity of a waveguide PC for air and PPy cover. (b) Transmittance intensity normalized with respect to the intensity outside the band gap region for air and PPy cover. 102

Figure 4.28 (a), (b) Measured transmittance and polarization response of a onedimensional Bragg filter without phase shift step patterned on a $2.7 \mu \mathrm{m}$-thick $\mathrm{Bi}_{0.8} \mathrm{Gd}_{0.2} \mathrm{Lu}_{2.0} \mathrm{Fe}_{5} \mathrm{O}_{12}$ film. The blue and magenta color data points show output polarization rotation of PC with PPy cover and PPy cover exposed to aqueous ammonia. Measurements are taken at magnetic field applied collinear to the waveguide axis. 104

Figure 4.29 (a), (b) Measured transmittance and polarization response of a onedimensional Bragg filter without phase shift step patterned on a $2.7 \mu \mathrm{m}$-thick $\mathrm{Bi}_{0.8} \mathrm{Gd}_{0.2} \mathrm{Lu}_{2.0} \mathrm{Fe}_{5} \mathrm{O}_{12}$ film. The blue and red color data points show output polarization rotation of PC with PPy cover and PPy cover exposed to methanol. Measurements are taken at magnetic field applied collinear to the waveguide axis. 105

Figure 4.30 An interferometric image of a Polypyrrole (PPy) thin film deposited on a silicon $(\mathrm{Si})$ substrate. 106

Figure $4.31 \Psi$ versus wavelength spectra for the experimental and the model fit for a Polypyrrole thin film deposited on a silicon substrate using ellipsometry. 107 


\section{List of Tables}

Table 3-1. Prism coupler measurements of wave guiding in the thin film slab for TE and TM input polarizations ...............................................................4

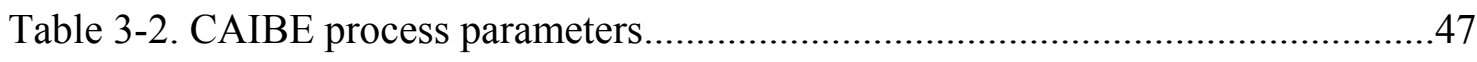

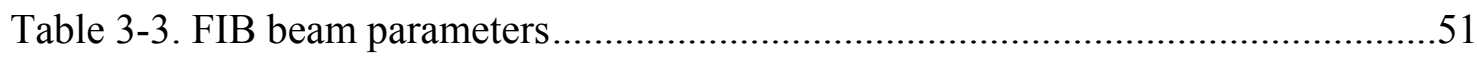

Table 4-1. Prism coupler measured refractive index data and the corresponding ..........71

Table 4-2. Refractive index and birefringence data for sample A ..............................76

Table 4-3. Refractive index and birefringence data for sample B ..............................77

Table 4-4. Refractive index and birefringence data for sample C .............................77

Table 4-5. Prism coupler measured index data for refractive index liquids used in

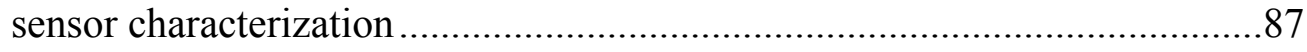

Table 4-6. Detection limit of various RI sensing technologies..................................98

Table A-1. Stokes parameters of the output light of a photonic crystal .....................120 


\section{ACKNOWLEDGEMENTS}

This work could not have been a success without the help and support of many people. First and foremost I would like to express my gratitude to Prof. Miguel Levy for giving me the opportunity to pursue my doctoral research under his guidance. He taught me how to conduct scientific research, how to approach complex problems and find solutions. I am truly indebted to him for helping me in every possible way throughout my $\mathrm{PhD}$ career. I would like to express my gratitude towards my dissertation advisory committee members, Prof. Brian Fick, Prof. Ranjit Pati, and Prof. Christopher Middlebrook for their time and input in my research. I also thank Prof. Kim Fook Lee and Prof. Claudio Mazzoleni who once served in my $\mathrm{PhD}$ advisory committee.

I gratefully acknowledge Physics department, Prof. Ravi Pandey, and the faculty members for their support, instruction, and financial assistance. Also I am thankful to physics department staff, Kathy, Andy and Marg for their help throughout these years.

I would like to express my gratitude to my parents and my two sisters for their love and support. My dear sisters Deepthi and Madu, you are the best sisters anybody could ever wish for, thank you for believing in me, encouraging me and always be there for me. I met my other half, Pradeep here in Houghton. He has been the most wonderful friend, colleague and husband. Your constant support, encouragement and love kept me going during the difficult times. Thank you so much for being a part of my life. Also I want to thank my son Aryan for bringing me the greatest joy in my life.

I received a lot of help from the previous and current members of Prof. Levy's research group while completing this work. Dr. Amir Jalali helped me with both theoretical analysis related to this work and with initial experiments. Dr Raghav Vanga and Dr. Xiaoyue Huang gave me the first lessons on optics tests and fabrication techniques used in Dr Levy's research group. Dr. Ziyou Zhou trained me to use the optical setup and Dr. Zhuoyuan $\mathrm{Wu}$ helped me in learning most of the micro and nano fabrication

techniques. Thank you very much for sharing your knowledge with me. It was xiii 
enjoyable to collaborate and work with Ding, Ashim and Jacob. I really appreciate their help in research and also in things out side of research. Special thanks to Jacob for improving the optics measurement set-up, it made my work efficient and convenient.

I would like to thank Dr. Vince Fratello for his research collaborations and the supply of high-quality LPE films. I am thankful to Owen Mills and William Knudsen for their help with the instruments in Materials Characterization \& Fabrication Facilities and Micro Fabrication Facilities at MTU. 


\section{Abbreviations}

$\begin{array}{ll}\text { RI } & \text { Refractive Index } \\ \text { MPC } & \text { Magneto-Photonic Crystal } \\ \text { PC } & \text { Photonic Crystal } \\ \text { PPy } & \text { Polypyrrole } \\ \text { TE } & \text { Transverse Electric } \\ \text { TM } & \text { Transverse Magnetic } \\ \text { FIB } & \text { Focused Ion Beam } \\ \text { SEM } & \text { Scanning Electron Microscopy } \\ \text { CAIBE } & \text { Chemically Assisted Ion Beam Etching } \\ \text { RCP } & \text { Right-Circularly Polarized } \\ \text { LCP } & \text { Left-Circularly Polarized } \\ \text { FDTD } & \text { Finite Difference Time Domain } \\ \text { GGG } & \text { Gadolinium Gallium Garnet }\left(\mathrm{Gd}_{3} \mathrm{Ga}_{5} \mathrm{O}_{12}\right) \\ \text { NPGS } & \text { Nano Pattern Generation System } \\ \text { LPE } & \text { Liquid Phase Epitaxy } \\ \end{array}$




\section{ABSTRACT}

Among the optical structures investigated for optical sensing purpose, a significant amount of research has been conducted on photonic crystal based sensors. A particular advantage of photonic crystal based sensors is that they show superior sensitivity for ultra-small volume sensing. In this study we investigate polarization changes in response to the changes in the cover index of magneto-optic active photonic band gap structures. One-dimensional photonic-band gap structures fabricated on iron garnet materials yield large polarization rotations at the band gap edges. The enhanced polarization effects serve as an excellent tool for chemical sensing showing high degree of sensitivity for photonic crystal cover refractive index changes.

The one dimensional waveguide photonic crystals are fabricated on single-layer bismuth-substituted rare earth iron garnet films $\left((\mathrm{Bi}, \mathrm{Y}, \mathrm{Lu})_{3}(\mathrm{Fe}, \mathrm{Ga})_{5} \mathrm{O}_{12}\right)$ grown by liquid phase epitaxy on gadolinium gallium garnet substrates. Band gaps have been observed where Bragg scattering conditions links forward-going fundamental waveguide modes to backscattered high-order waveguide modes. Large near-bandedge polarization rotations which increase progressively with backscattered-mode order have been experimentally demonstrated for multiple samples with different composition, film thickness and fabrication parameters. Experimental findings are supported by theoretical analysis of Bloch modes polarization states showing that large near stop-band edge rotations are induced by the magneto-photonic crystal.

Theoretical and experimental analysis conducted on polarization rotation sensitivity to waveguide photonic crystal cover refractive index changes shows a monotonic enhancement of the rotation with cover index. The sensor is further developed for selective chemical sensing by employing Polypyrrole as the photonic crystal cover layer. Polypyrrole is one of the extensively studied conducting polymers for selective analyte detection. Successful detection of aqueous ammonia and methanol has been achieved with Polypyrrole deposited magneto-photonic crystals. 


\section{INTRODUCTION}

In the last three decades there has been growing interest on optical sensors research due to their vast applications in medical, health care, environment and in national security sectors $[1,2,3,4]$. Optical sensors are immune to electromagnetic interference and capable of multi analyte detection in addition to provide low cost sensing solutions for wide verity of analytes [1,4]. One of the other attractive feature of optical sensing is that the detection signal is related to sample concentration or surface density instead of total sample mass, which enables ultra-small volume sensing [1].

The main elements of an optical sensor consist of the analyte, the transduction signal and signal processing, with the transduction signal essentially being an optical signal. The optical signal could be due to absorption, luminance, or other optical properties such as refractive index (RI) and reflectivity. In this work, an optical sensor based on refractive index detection has been developed and demonstrated. Although many onchip integrated optical sensors have been explored for RI-based detection, magnetophotonic-crystal-enhanced polarization effects for optical sensing have not being explored so far [1-10]. This dissertation research investigates optical sensing based on polarization rotation sensitivity to magneto-photonic crystal (MPC) cladding in waveguide configurations.

\subsection{Photonic crystals for optical sensing}

Among the optical structures investigated for optical sensing, a significant amount of research has been conducted on photonic crystal based sensors [5, 7-9]. A photonic crystal (PC) is a periodic dielectric structure with a periodicity of the order of the optical wavelength. Photonic band gaps are formed where propagation of certain frequencies of light is blocked due to destructive interference by the periodic arrangement of materials. These band gap structures have shown many applications in opto-electronics devices which include many optical sensor devices. 
(a)

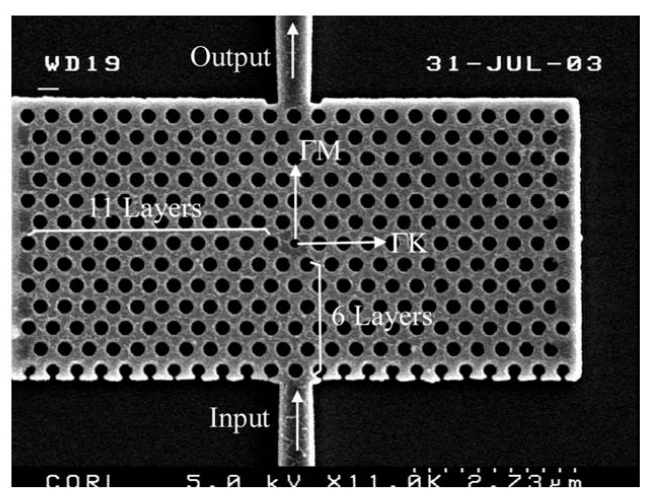

(b)

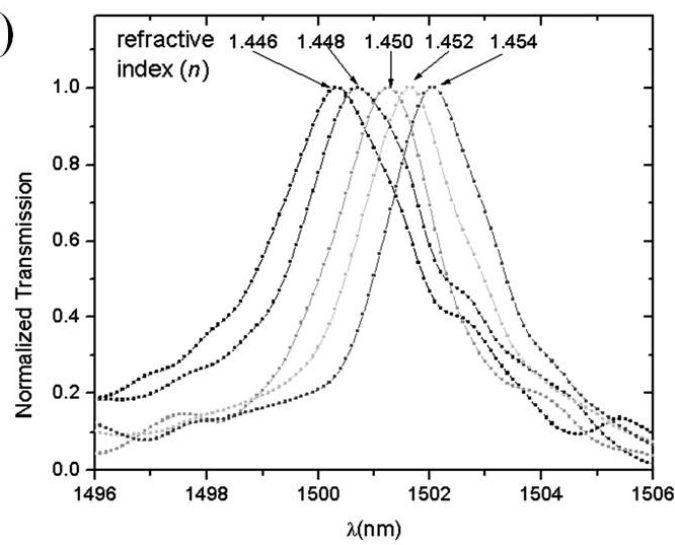

(c)

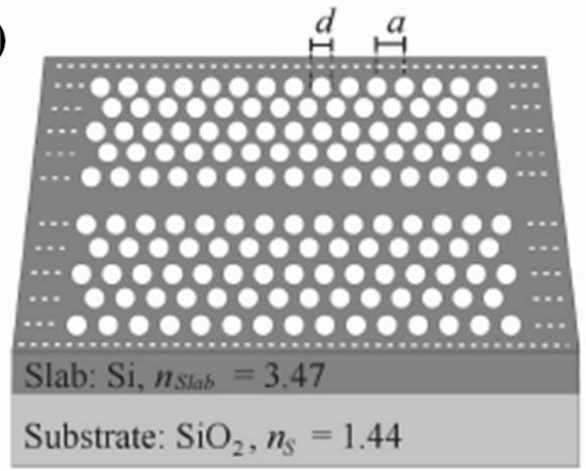

(d)

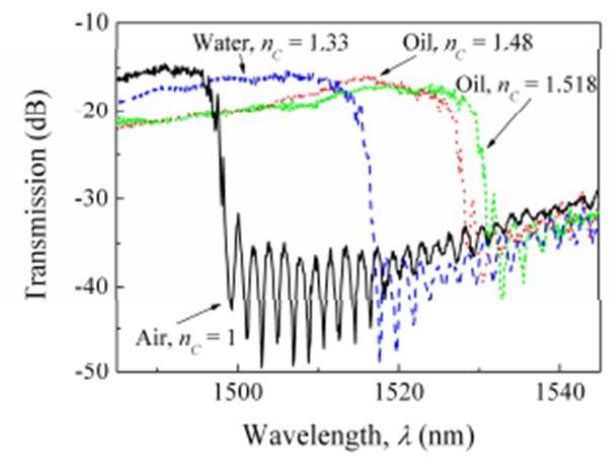

Figure 1.1 (a),(b) Scanning electron microscope view of a photonic crystal microcavity integrated with two ridge waveguides, normalized transmission spectra of the photonic crystal microcavity [shown in (a)] with five different ambient refractive indices ranging from $n=1.446$ to $n=1.454$. (c),(d) Photonic crystal waveguide (PC-WG) on SOI wafer, measured transmittance spectra from the fabricated PC-WG for four different cover solutions $[5,8]$. (Reprinted from refs. 5 and 8, permission details in Appendix B)

A resonant cavity or a defect can be introduced to the photonic structure by disturbing the periodicity of the structure leading to a resonant or defect mode in the band gap. PCs both with and without resonant cavities have been researched by other authors for RI based optical sensing [1, 5, 7-9]. The detection mechanisms of these types of sensors rely on shifts induced on a resonant wavelength or on the spectral location of a 
band gap in response to refractive index changes. Figure 1.1 shows two different photonic crystal-based RI sensor devices and their characterization results.

The photonic crystal structures can be fabricated at a nano-scale, allowing manipulation of light in very small spaces, a feature very important for ultra small volume sensing. Also parallel sensing can be achieved by having array of photonic crystals in a single microchip.

\subsection{Magneto-photonic crystal optical sensors}

The author's work presents a PC based optical sensor in magnetic (gyrotropic) materials. In the presence of an applied magnetic field, the plane of polarization of the light waves can be rotated as they propagate through gyrotropic media. Hence photonic crystals fabricated on gyrotropic media provide functional phonic band gaps with additional polarization effects in the output light. Previous research done in professor Levy's research group has shown that photonic crystals fabricated on gyrotropic media yield significantly enhanced polarization rotations [11-16]. Further theoretical analysis in polarization studies predicts that polarization rotation is highly sensitive to the PC cover refractive index [12-16]. Hence polarization rotation as a response to the PC cladding index changes could be utilized as a sensitive tool for optical sensing. The first half of this dissertation research involves developing a magneto-photonic crystal polarization-rotation-based optical-sensing technique for RI detection. The sensor calibration was done by modifying the PC cladding by applying refractive index liquids of known index on the PC. The second half of the research investigates the development of the sensor for toxic substance detection. Polypyrrole (PPy), a conducting polymer which is sensitive to many toxic and nontoxic substances was used as modified PC cover layer [3,10,17-19]. It is reported that PPy undergoes reversible or irreversible reactions when exposed to substances such as ammonia $\left(\mathrm{NH}_{3}\right)$, hydrogen sulfide $\left(\mathrm{H}_{2} \mathrm{~S}\right)$, carbon monoxide (CO), alcohols, etc [3,10,18]. The interactions with these substances 
cause PPy to change its electrical conductivity and hence its refractive index. The author has successfully demonstrated detection of aqueous ammonia and methanol using modified PPy-PC cover layers [17]. Figure 1.2 shows the schematic representation of the developed sensing device.

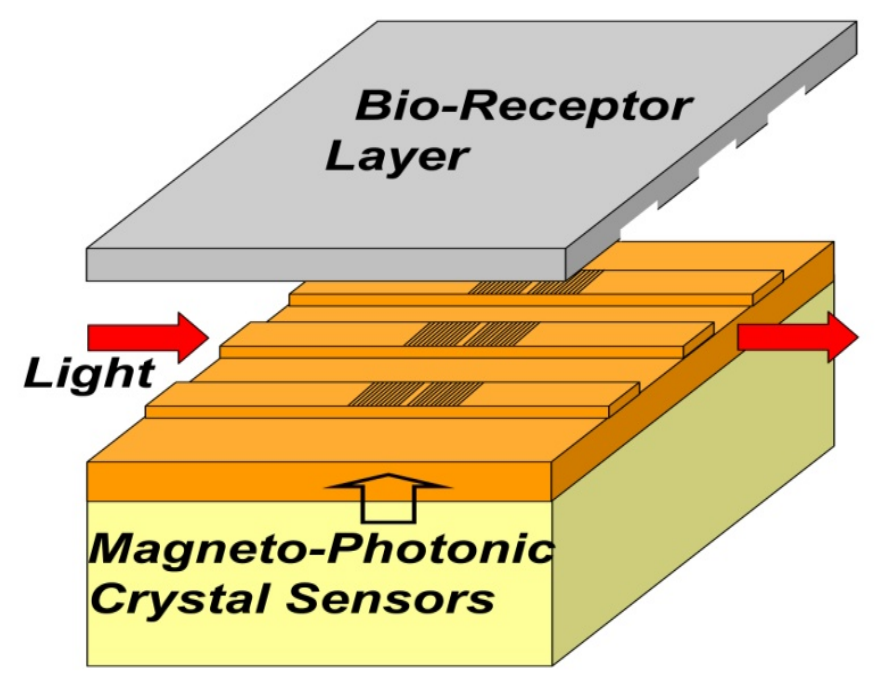

Figure 1.2 Magneto-photonic crystal sensor with a receptor layer applied on the grating structure.

\subsection{Organization of the dissertation}

This dissertation is divided into five chapters. Chapter two provides related theoretical background on optical waveguide theory, magnetic materials and the magneto-optic effect, photonic crystals, wave propagation in magneto-optic media and band gap formation, conjugated polymer Polypyrrole and its use for chemical sensing.

Chapter three gives systematic experimental steps for fabrication and characterization of the sensing device. This includes optical characterization of garnet 
thin films, fabrication of ridge waveguides and photonic crystals and their optical characterization, sensor device characterization and development for aqueous ammonia and methanol detection.

Chapter four presents all the theoretical and experimental results and related discussion.

Chapter five summarizes the work and presents conclusions based on this work. The chapter ends with a discussion on improvements to the sensor and proposed future work. 


\section{THEORETICAL BACKGROUND}

\subsection{Planar dielectric waveguides}

A planar waveguide is a dielectric slab surrounded by lower refractive index media. The guiding or core layer is sandwiched between an upper layer, called cover layer and a lower layer or substrate. The light is mostly confined in the higher index guiding layer by total internal reflection. The basic slab waveguide structure is shown in Fig. 2.1. $\mathrm{n}_{\mathrm{c}}$, $\mathrm{n}_{\mathrm{f}}$ and $\mathrm{n}_{\mathrm{s}}$ are refractive indices of cover, film (guiding layer) and substrate, respectively.

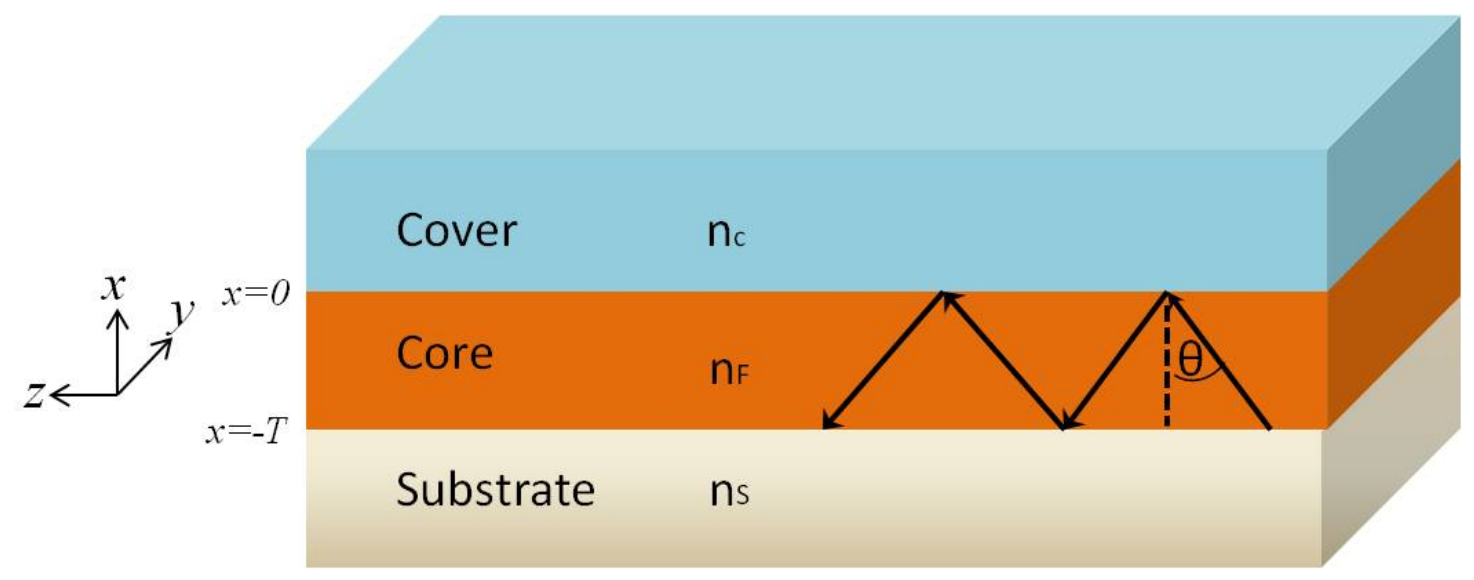

Figure 2.1 Basic slab waveguide structure

To achieve waveguiding in the structure, the guiding layer thickness $\mathrm{T}$ should be above a critical thickness which is of the order of the wavelength and also refractive indices satisfy $n_{f}>n_{s}>n_{c}$. In 2-D slab waveguides, the light is confined only in the $x$ direction where as in 3-D waveguides light confined in both $x$ and $y$ directions. 


\subsubsection{Guided modes}

Guided modes in the slab waveguides by total internal reflection can be understood by ray-optics concepts. Critical angles for total internal reflection at upper and lower interfaces are, respectively,

$$
\begin{gathered}
\theta_{c}=\sin ^{-1}\left(\frac{n_{c}}{n_{f}}\right) \\
\theta_{s}=\sin ^{-1}\left(\frac{n_{s}}{n_{f}}\right)
\end{gathered}
$$

Consider a light beam incident on the waveguide interface as shown in Fig. 2.1. When $\theta_{s}<\theta<90^{\circ}$, and assuming $\theta_{c}<\theta_{s}$, light is fully confined in the guiding layer by total internal reflection both at the upper and lower interfaces, which corresponds to a guided mode. If $\theta_{c}<\theta<\theta_{s}$, the light is totally reflected at the upper interface while leaking out at the lower interface; this corresponds to a substrateradiation mode. When $\theta<\theta_{c}$, the light radiates into both cover and substrate corresponding to a substrate-clad radiation mode.

\subsubsection{Effective or mode index}

Guided modes in a waveguide-geometry are characterized by their propagation constants. The plane wave propagation constant is defined as $k_{0} n_{f}$ where $k_{0}=\frac{2 \pi}{\lambda}$ and $\lambda$ is the light wavelength in free space. Wave vector diagram is shown in Fig 2.2.

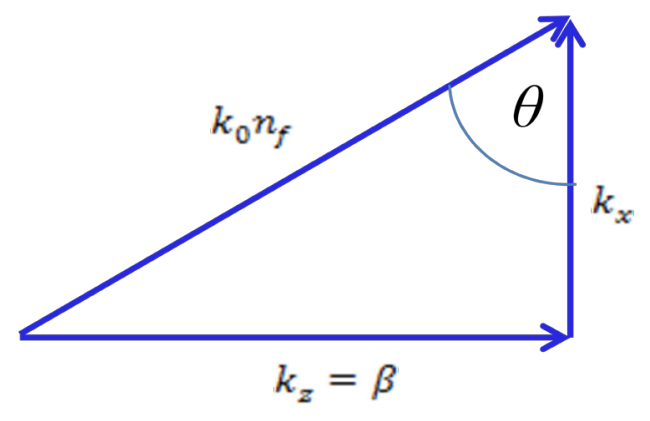

Figure 2.2 Wave vector diagram 
The propagation constants along the $x$ and $z$ directions can be written,

$$
\begin{aligned}
& k_{x}=k_{0} n_{f} \cos \theta \\
& k_{z}=k_{0} n_{f} \sin \theta=\beta
\end{aligned}
$$

The effective index $N$ is defined as,

$$
\begin{aligned}
& \beta=k_{0} N \\
& N=n_{f} \sin \theta
\end{aligned}
$$

This is the actual guided mode index of the wave propagated in $\mathrm{z}$ direction. The range of $N$ can vary

$$
n_{s}<N<n_{f}
$$

The number of modes supported by the waveguide depends on the film thickness, the wavelength and the indices of the three layers. For given wavelength and waveguide structure, only the fundamental mode can exist right above the cut off thickness. If the thickness is increased, more than one mode is allowed.

The first four waveguide modes of a $2.7 \mu \mathrm{m}$ thick ridge waveguide structure calculated by beam propagation simulation tool (BeamPROP) by RSoft, a commercial photonic and network design software are shown in Fig. 2.3. A thickness of $2.7 \mu \mathrm{m}$ is typical for the waveguides used in the experimental work for this dissertation. The refractive index of the cover, film and substrate are 1.00, 2.31, and 1.95 respectively. The calculated mode indices of the first four modes are 2.3028, 2.2591, 2.1861 and 2.0818. As stated above in equation 2.7, the effective indices of the waveguide modes take values between the substrate and film indices, 1.95 and 2.31, respectively. 

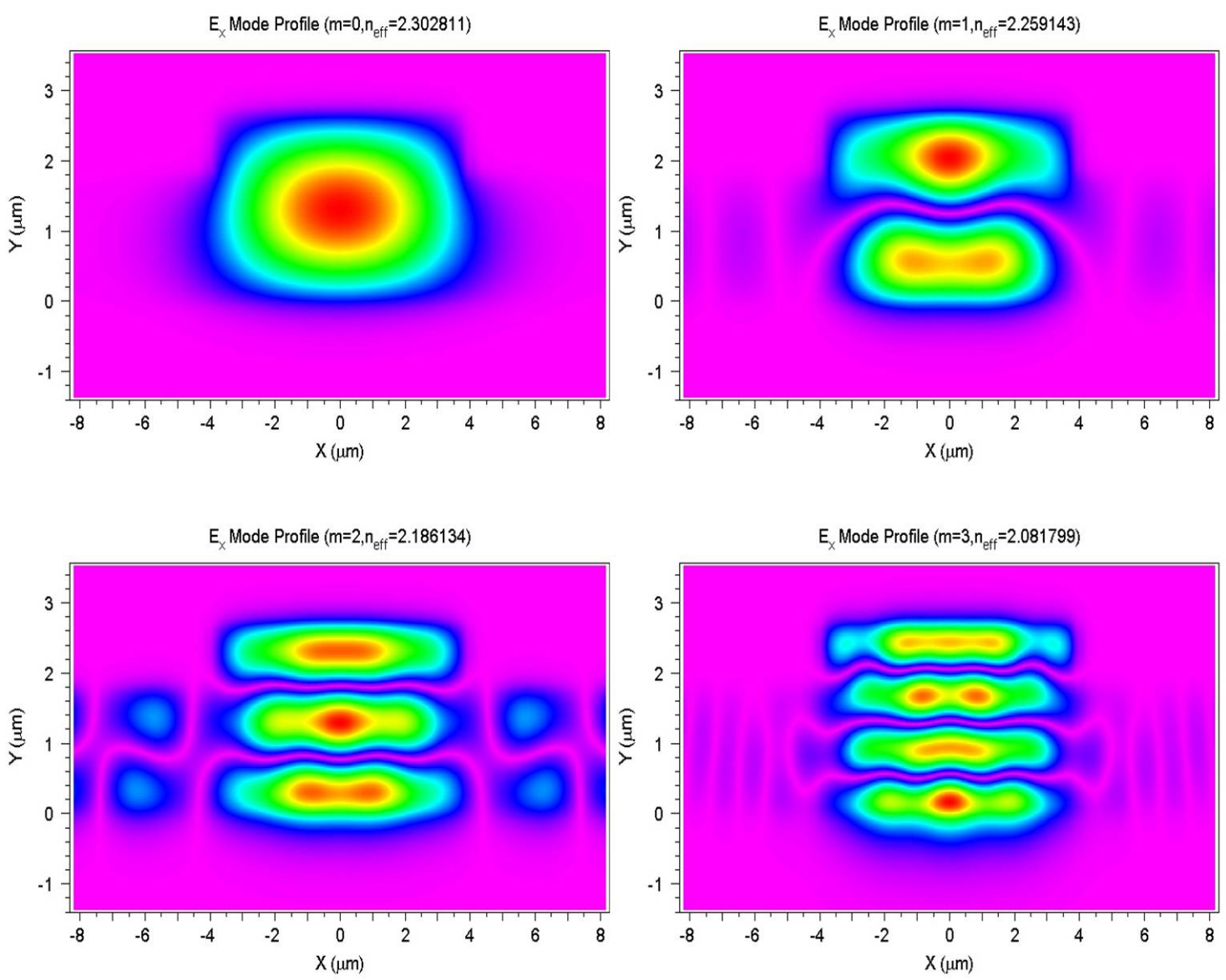

Figure 2.3 First four waveguide modes supported by a $2.71 \mu \mathrm{m}$ thick ridge waveguide. The effective indices of the modes vary between the substrate index 1.95 and film index 2.31 .

\subsubsection{Waveguide Theory}

Maxwell equations in homogenous lossless media are given by

$$
\begin{gathered}
\nabla \times E=-\mu_{0} \frac{\partial H}{\partial t} \\
\nabla \times H=-\varepsilon_{0} n^{2} \frac{\partial E}{\partial t}
\end{gathered}
$$


Where $n$ is the refractive index, $\varepsilon_{0}$ is the dielectric permittivity and $\mu_{0}$ the magnetic permeability of free space.

For a plane wave propagating along the $z$-direction with propagation constant $\beta$, the spatial dependence of the electromagnetic wave can be written as follows.

$$
\begin{aligned}
& E=E(x, y) \cdot e^{j(\omega t-\beta z)} \\
& H=H(x, y) \cdot e^{j(\omega t-\beta z)}
\end{aligned}
$$

With angular frequency $\omega=\frac{2 \pi c}{\lambda}$ and light velocity in free space $c=\frac{1}{\sqrt{\varepsilon_{0} \mu_{0}}}$

For the 2-D slab waveguide shown in Fig. 2.1, the electromagnetic fields are independent of $y$. Given that $\frac{\partial}{\partial t}=j \omega, \frac{\partial}{\partial z}=-i \beta$ and $\frac{\partial}{\partial y}=0$, Eqs. 2.8 and 2.9 generate two different modes with mutually orthogonal polarization states. One is the transverse-electric (TE) mode which consists of field components $E_{y}, H_{x}$, and $H_{z}$. The other is the transverse-magnetic (TM) mode with $E_{x}, H_{y}$, and $E_{z}$.

For the TE mode, the following relations are satisfied:

$$
\begin{gathered}
\frac{\partial^{2} E_{y}}{\partial x^{2}}+\left(k_{0}{ }^{2} n^{2}-\beta^{2}\right) E_{y}=0 \\
\left\{\begin{array}{c}
H_{x}=-\frac{\beta}{\omega \mu_{0}} E_{y} \\
H_{z}=-\frac{\beta}{j \omega \mu_{0}} \frac{\partial E_{y}}{\partial x}
\end{array}\right\}
\end{gathered}
$$

The TM mode satisfies the following relations:

$$
\frac{\partial^{2} H_{y}}{\partial x^{2}}+\left(k_{0}^{2} n^{2}-\beta^{2}\right) H_{y}=0
$$




$$
\left\{\begin{aligned}
E_{x} & =\frac{\beta}{\omega \varepsilon_{0} n^{2}} H_{y} \\
E_{z} & =\frac{1}{j \omega \varepsilon_{0} n^{2}} \frac{\partial H_{y}}{\partial x}
\end{aligned}\right\}
$$

Field solutions for the TE mode can be obtained from Eq. 2.11

$$
\left\{\begin{array}{cr}
E_{y}=E_{c} \exp \left(-\gamma_{c} x\right), & x>0 \text { (cover) } \\
E_{y}=E_{f} \cos \left(k_{x} x+\phi_{c}\right), & -T<x<0 \text { (guiding layer) } \\
E_{y}=E_{s} \exp \left\{\gamma_{s}(x+T)\right\}, & x<-T \text { (substrate) }
\end{array}\right\}
$$

where the propagation constants are expressed in terms of the effective index $N$,

$$
\gamma_{c}=k_{0} \sqrt{N^{2}-n_{c}^{2}}, \quad k_{x}=k_{0} \sqrt{n_{f}^{2}-N^{2}} \quad, \quad \gamma_{s}=k_{0} \sqrt{N^{2}-n_{s}^{2}}
$$

The tangential field components $E_{y}$ and $H_{z}$ are continuous at the boundaries. Therefore applying these boundary conditions in Eq.2.15 and making use of Eq. 2.12 for $H_{z}$ we get,

$$
\left.\begin{array}{r}
E_{c}=E_{f} \cos \phi_{c} \\
\tan \phi_{c}=\frac{\gamma_{c}}{k_{x}}
\end{array}\right\}
$$

The eigenvalue equation for TE mode is obtained by eliminating arbitrary coefficients in 2.17 and 2.18 ,

$$
k_{x} T=(m+1) \pi-\tan ^{-1}\left(\frac{k_{x}}{\gamma_{s}}\right)-\tan ^{-1}\left(\frac{k_{x}}{\gamma_{c}}\right)
$$


Where $\mathrm{m}=0,1,2 \ldots$ gives the mode order.

Similarly the TM mode eigenvalue equation can be obtained from Eq. 2.13 and the application of the boundary conditions to the fields,

$$
k_{x} T=(m+1) \pi-\tan ^{-1}\left\{\left(\frac{n_{s}}{n_{f}}\right)^{2}\left(\frac{k_{x}}{\gamma_{s}}\right)\right\}-\tan ^{-1}\left\{\left(\frac{n_{c}}{n_{f}}\right)^{2}\left(\frac{k_{x}}{\gamma_{c}}\right)\right\}
$$

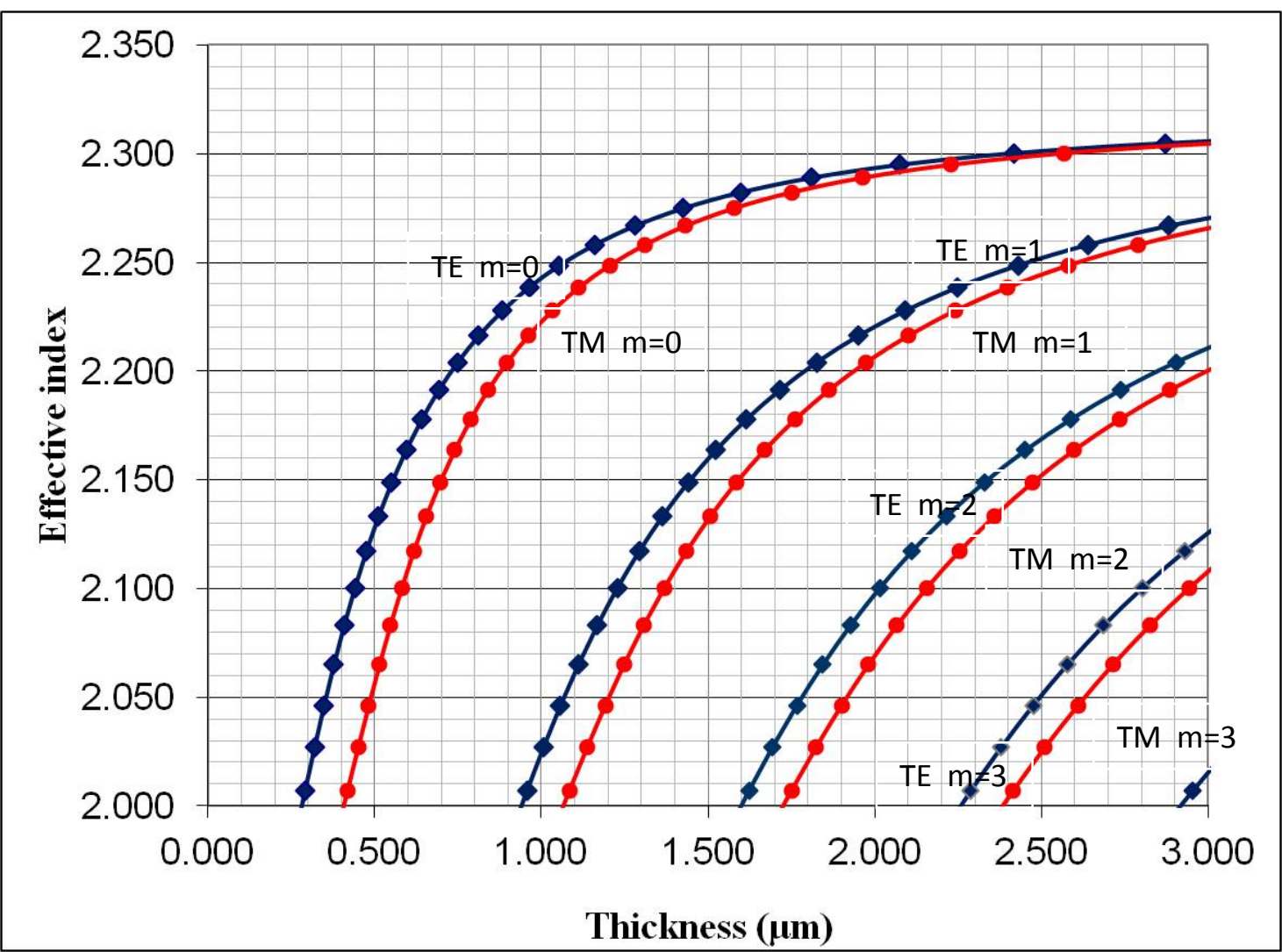

Figure 2.4 Calculated TE and TM waveguide modes for a garnet film with refractive index 2.31 at a wavelength of $1543 \mathrm{~nm}$.

The dispersion characteristics of guided modes can be evaluated by solving Eqs. 2.19 and 2.20 numerically for known waveguide parameters. Figure 2.4 shows the 
calculated TE and TM effective index mode values for a magnetic garnet film with refractive index 2.31. According to the graph, a $2.5 \mu \mathrm{m}$-thick film supports four TE and TM waveguide modes.

\subsection{Linear birefringence}

Linear birefringence in optical waveguides refers to the difference between the refractive indices of TE and TM modes [21]. There are three effects which give rise to linear birefringence in epitaxial garnet films: geometrical or shape birefringence, stressinduced birefringence, and growth-induced birefringence [21,22]. Geometrical birefringence is observed in any film whose thickness is comparable to the wavelength of the light. In multimode waveguides, there is a different birefringence for each pair of modes and the magnitude is larger for higher order modes. Shape birefringence increases as the film thickness decreases and with increasing optical wavelength. The linear birefringence variation with film thickness can be seen Fig. 2.4. Stress-induced birefringence is observed in films where the lattice constant of the substrate is different from that of the film. The stress birefringence is independent of thickness and wavelength. Growth induced birefringence is associated with the film growth process. The physical origin of the growth induced birefringence is associated with the deformation of the atomic environment or break of the cubic symmetry of the crystal. In the Bismuth substituted iron garnets, large $\mathrm{Bi}^{3+}$ ions seems to deform the microscopic structure of the crystal leading to the growth induced birefringence in the film [23]. The magnitude and sign of these effects can vary, depending on the garnet composition, crystal orientation, and growth parameters. The growth-induced birefringence is independent of thickness or wavelength and it can be minimized by growing at the highest possible temperature, or by high temperature annealing in flowing nitrogen atmosphere [22]. 


\subsection{Magnetic materials}

For a given material, the relationship between the applied magnetic field $H$, the magnetic induction $B$ and magnetization $M$ of the material is given,

$$
B=\mu_{0}(H+M)
$$

where $\mu_{0}$ the permeability of the free space given by $4 \pi \times 10^{-7} H . m^{-1}$. The units of $H$ and $M$ are Amperes per meter $(\mathrm{A} / \mathrm{m})$ and of $B$ weber $/ \mathrm{m}^{2}$ or Tesla $(T) . M$ is a property of the material. Material magnetization is determined by the magnetic moments of the individual atoms or molecules, and their exchange and dipole moment interactions.

The magnetic susceptibility $\chi$ is defined as the ratio of the magnetization $M$ with the applied field $H$ in the limit of small applied fields,

$$
\chi=\frac{M}{H}
$$

The permeability $\mu$ of a material is given by

$$
\mu=\frac{B}{H}
$$

From Eqs. 2.21, 2.22 and 2.23 we get,

$$
\mu=\mu_{0}(1+\chi)
$$

The magnetic moment of a free atom stems from the orbital angular momenta of the electrons about the nucleus, the spin angular momentum of each electron, the change in orbital angular momenta induced by an applied magnetic field, and the nuclear magnetic moment. An atom or ion is considered to be magnetic if it possesses a permanent magnetic dipole moment. Diamagnetism results from the change in orbital angular momentum of the electrons induced by an applied magnetic field. Electronic paramagnetism is due to electronic spin and orbital angular momenta. Diamagnetic materials have negative magnetic susceptibility, whereas paramagnetic materials have positive susceptibility. In a paramagnetic material the dipole moments are randomly 
oriented in the absence of a magnetic field, whereas in antiferromagnetic materials the dipole moments are oriented antiparallel to each other. In ferromagnetic materials, the magnetic dipole moments are oriented in the same direction as a result of the exchange interaction. Ferrimagnetic materials consist of dipoles arranged antiparallel to each other but of different magnitude so that the material has a net magnetic moment. Hence ferromagnetic and ferrimagnetic materials have nonzero resultant magnetic moments. Schematic depictions of the arrangement of the magnetic dipoles in different magnetic materials are shown Fig. 2.5 [24,25].

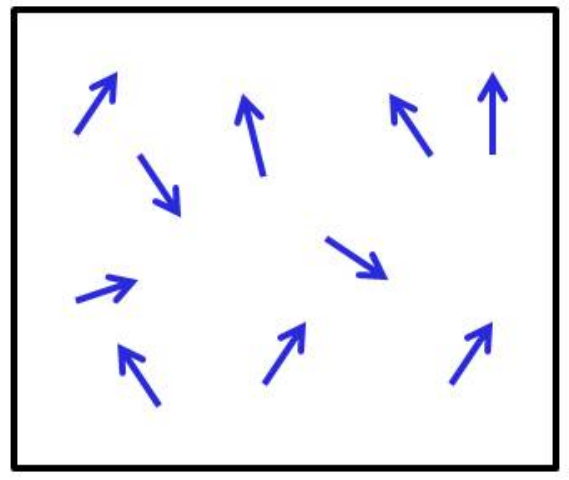

Paramagnetic

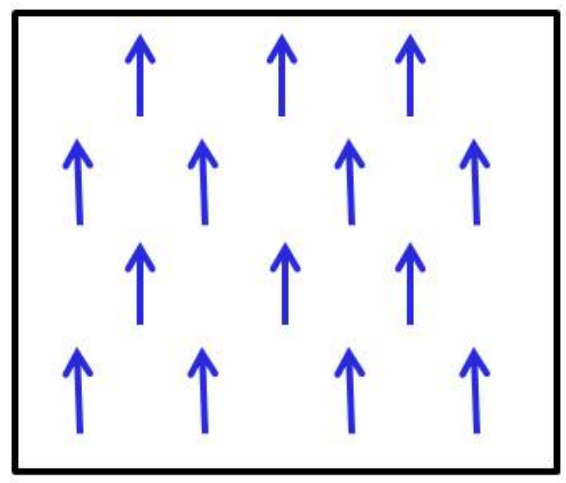

Ferromagnetic

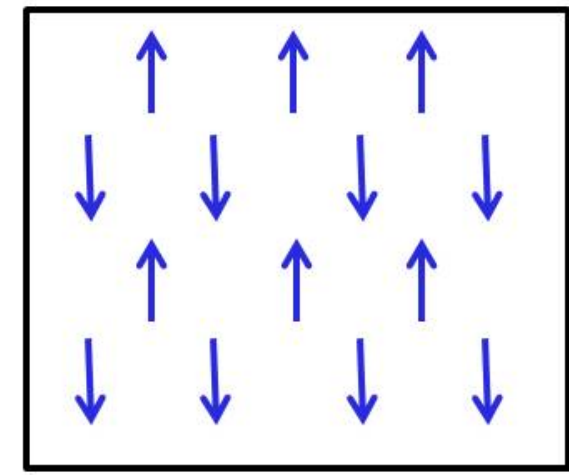

Antiferromagnetic

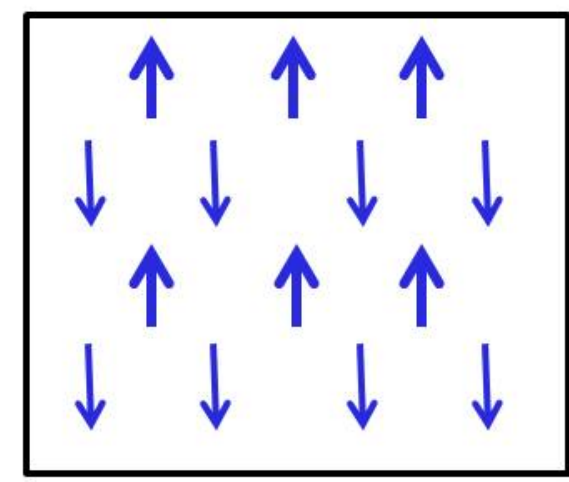

Ferrimagnetic

Figure 2.5 Magnetic dipole arrangements in magnetic materials 


\subsubsection{The magneto-optic effect}

Magneto-optic effects consist of a number of phenomena in which electromagnetic waves interact with magnetized materials. These effects can arise when light is transmitted through or reflected from the magnetized materials. Faraday rotation is one of the important transmission magneto-optic effects where the plane of polarization of light is rotated while propagating through the material. The Faraday effect is observed in both magnetic and non magnetic materials under an applied magnetic field [24].

\subsubsection{Faraday effect}

The Faraday effect (optical gyrotropy) is a magneto-optical effect discovered by Michael Faraday in 1845 . When linearly polarized light propagates through a transparent dielectric material which is magnetized parallel to the propagation direction, the plane of polarization of the light gets rotated through an angle $\theta_{F}$. The angle of rotation $\theta_{F}$, called Faraday rotation angle is proportional to the path length $d$ of the light in the material. If the magnetization direction is reversed the sense of rotation will be reversed. However if the magnetization direction is the same and the direction of propagation is reversed, the sense of rotation will be the same as in the original forward direction, resulting in accumulated rotation [24]. This is due to the nonreciprocal character of the Faraday effect, as shown in Fig. 2.6.

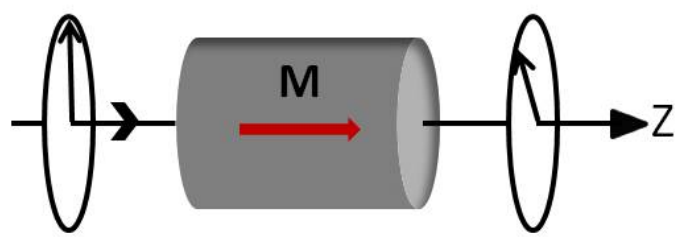

(a)

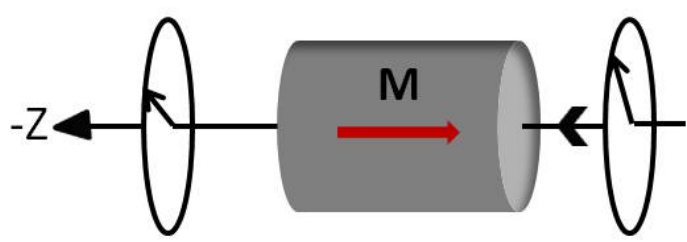

(b)

Figure 2.6 (a) Geometry of the Faraday effect. (b) Non-reciprocal character of the Faraday effect. 
When a magnetic material is magnetized parallel to the direction of propagation the dielectric tensor $\varepsilon$ becomes asymmetric as shown in 2.25. Here $j=\sqrt{-1}$ and $g$ is the gyrotropy parameter. At optical frequencies the relative permeability $\mu=1$ because the time dependence of the magnetization is neglegible in this light frequency range [20, $25]$.

$$
\varepsilon=\left(\begin{array}{ccc}
\varepsilon_{\perp} & -j g & 0 \\
j g & \varepsilon_{\perp} & 0 \\
0 & 0 & \varepsilon_{\|}
\end{array}\right)
$$

A beam of linearly polarized light entering into magnetized-lossless media at normal incidence decomposes into right-circularly polarized (RCP) and left-circularly polarized (LCP) normal mode components. The electric field vectors of the two circular polarized light components can be written,

$$
E_{ \pm}=E_{0} e^{ \pm j \omega t}
$$

The propagation constants are

$$
\begin{aligned}
& \beta_{+}=\omega \sqrt{\varepsilon_{0} \mu_{0} \varepsilon_{+}} \\
& \beta_{-}=\omega \sqrt{\varepsilon_{0} \mu_{0} \varepsilon_{-}}
\end{aligned}
$$

Where

$$
\begin{aligned}
& \varepsilon_{+}=\varepsilon_{\perp}-g \\
& \varepsilon_{-}=\varepsilon_{\perp}+g
\end{aligned}
$$

The refractive indices of the two polarized states in the medium are then given by $n_{ \pm}=$ $\sqrt{ }\left(\varepsilon_{\perp} \pm g\right)$. Hence they propagate in the media with different speeds and each emerges 
with a different phase at the output end. The plane of polarization of the recombined light is rotated by an angle $\theta_{F}$ given by,

$$
\theta_{F}=\frac{\Delta \varphi}{2}=\frac{1}{2}\left(\beta_{-}-\beta_{+}\right) d
$$

where $\Delta \varphi$ is the phase difference between the two circular polarized components, $d$ is the sample length. With equations 2.27 and 2.28 and with the condition $\varepsilon_{1} \gg g$, equation 2.29 becomes,

$$
\theta_{F}=\frac{\omega}{2} \sqrt{\frac{\mu_{0} \varepsilon_{0}}{\varepsilon_{\perp}}} \cdot g \cdot d
$$

In diamagnetic and paramagnetic materials $g$ is proportional to the applied magnetic field $H$, hence Faraday rotation becomes,

$$
\theta_{F}=V H d
$$

where $V$ is the Verde constant. For a ferromagnetic material $\theta_{F}$ is given by,

$$
\theta_{F}=F\left(\frac{M}{M_{S}}\right) d
$$

where $M s$ is the saturation magnetization and $F$ is the Faraday rotation coefficient (degrees per centimeter) [20, 25].

The presence linear birefringence in the material produces elliptically polarized light upon propagating through magnetized materials. The ellipticity depends on the magnitude of the linear birefringence. 


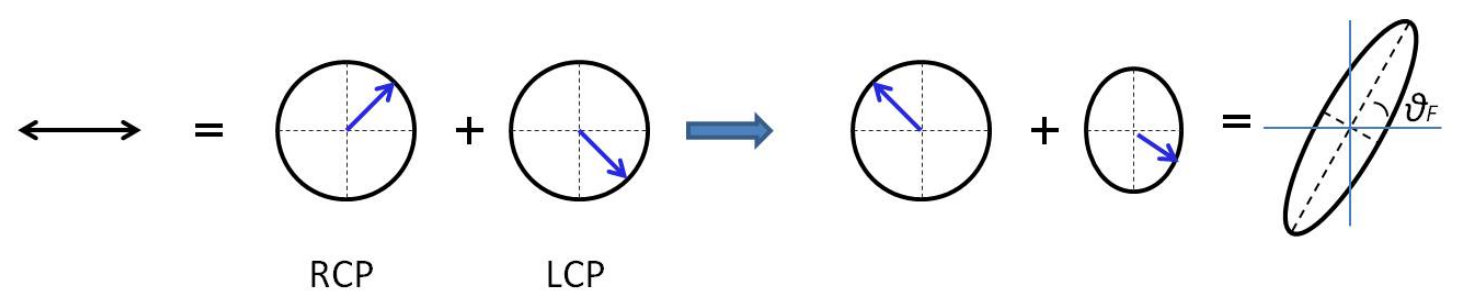

Figure 2.7 Faraday rotation in media with linear birefringence.

\subsection{Elliptical birefringence and polarization rotation}

Elliptical birefringence refers to the difference in refractive index between elliptically polarized normal modes. The simultaneous presence of linear birefringence and optical gyrotropy in the medium leads to orthogonal elliptically polarized normal modes. $[27,28]$ These elliptically polarized modes propagate through the medium with different refractive indices and acquire a phase difference. Therefore the polarization state of the linearly polarized light incident normally to the birefringent magneto-optic media becomes elliptically polarized and gets rotated through an angle $\theta$. The Faraday rotation in non-birefringent crystals increases linearly with path length of the light propagation. However the presence of linear birefringence suppresses the Faraday rotation and degrades it from linear polarization, resulting in elliptical polarization. The term "polarization rotation" is used to describe the rotation of the semi-major axis of the elliptical polarization (shown in Fig. 2.8) as a consequence of the combination of Faraday rotation and linear birefringence which is different from the pure Faraday rotation angle $\theta_{F}$. 


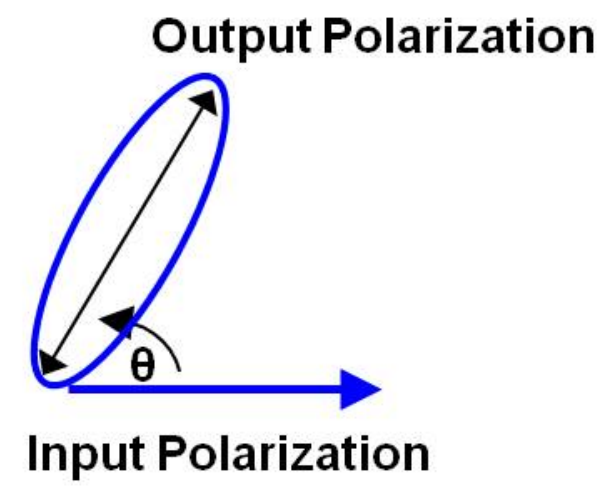

Figure 2.8 Linearly polarized light converted in to elliptically polarized light and rotated through an angle $\theta$ when propagated through birefringent magneto-optic media.

Normal mode propagation and polarization rotation in birefringent magneto optic media can be understood as follows [27]. In a lossless medium, let the Faraday rotation per unit length for the medium be denoted by $\alpha$ and the linear birefringence retardation per unit length $(2 \pi / \lambda)\left(n_{y}-n_{x}\right)$ be denoted by $\beta$ respectively. Then the propagation matrices for the electric field amplitude can be written, as

$$
F(z)=\left[\begin{array}{cc}
\cos \alpha z & \sin \alpha z \\
-\sin \alpha z & \cos \alpha z
\end{array}\right]
$$

and

$$
B(z)=\exp \left(-i \frac{2 \pi}{\lambda} \bar{n} z\right)\left[\begin{array}{cc}
\exp \left(\frac{i \beta z}{2}\right) & 0 \\
0 & \exp \left(\frac{-i \beta z}{2}\right)
\end{array}\right]
$$

where

$$
\bar{n}=\left(\sqrt{\varepsilon_{x x}}+\sqrt{\varepsilon_{y y}}\right) / 2
$$

$\varepsilon_{x x}$ and $\varepsilon_{y y}$ are the diagonal components of the dielectric tensor of the medium. The combined effect of both linear and circular transformation on the electric field amplitude vector $E(z)=\left[\begin{array}{l}E_{x} \\ E_{y}\end{array}\right]$ over an infinitesimal distance $\delta z$ can be written as, 


$$
F(\delta z) B(\delta z)=B(\delta z) F(\delta z)=\left(1-i \frac{2 \pi}{\lambda} \bar{n} \delta z\right)\left[\begin{array}{cc}
1+i \frac{\beta \delta z}{2} & \alpha \delta z \\
-\alpha \delta z & 1-i \frac{\beta \delta z}{2}
\end{array}\right]
$$

And

$$
E(z+\delta z)-E(z)=\left\{\left(-i \frac{2 \pi}{\lambda} \bar{n}\right)\left[\begin{array}{cc}
i \frac{\beta}{2} & \alpha \\
-\alpha & -i \frac{\beta}{2}
\end{array}\right]\right\} E(z) \delta z
$$

By integrating the matrix differential equation, the transformation matrix $P(z)$ for the propagation of polarized light in medium with combined linear and circular birefringence can be obtained.

$$
P(z)=\exp \left(-i \frac{2 \pi}{\lambda} \bar{n} z\right) \times\left[\begin{array}{cc}
\cos (b z)+i\left(\frac{\beta}{2 b}\right) \sin (b z) & \left(\frac{\alpha}{b}\right) \sin (b z) \\
-\left(\frac{\alpha}{b}\right) \sin (b z) & \cos (b z)-i\left(\frac{\beta}{2 b}\right) \sin (b z)
\end{array}\right]
$$

where $b=\sqrt{\alpha^{2}+\frac{\beta^{2}}{4}}$

To obtain the normal modes of the medium, we solve the eigenvalue equation,

$$
\begin{array}{r}
\exp \left(-i \frac{2 \pi}{\lambda} \bar{n} z\right) \times\left[\begin{array}{cc}
\cos (b z)+i\left(\frac{\beta}{2 b}\right) \sin (b z) & \left(\frac{\alpha}{b}\right) \sin (b z) \\
-\left(\frac{\alpha}{b}\right) \sin (b z) & \cos (b z)-i\left(\frac{\beta}{2 b}\right) \sin (b z)
\end{array}\right] E(z)= \\
\chi \exp \left(-i \frac{2 \pi}{\lambda} \bar{n} z\right) E(z)
\end{array}
$$

\subsection{8}

$\chi$ is a scalar quantity determined by equating the following determinant to zero, 


$$
\operatorname{det}\left[\begin{array}{cc}
\cos (b z)+i\left(\frac{\beta}{2 b}\right) \sin (b z)-\chi & \left(\frac{\alpha}{b}\right) \sin (b z) \\
-\left(\frac{\alpha}{b}\right) \sin (b z) & \cos (b z)-i\left(\frac{\beta}{2 b}\right) \sin (b z)-\chi
\end{array}\right]
$$

The solution to the determinant is,

$$
\chi=\left[\cos (b z) \pm \sqrt{\cos ^{2}(b z)-1}\right]=[\cos (b z) \pm \sin (b z)=\exp ( \pm i b z)]
$$

Hence the normal modes phase factors are, $\exp \left[-i\left(\frac{2 \pi}{\lambda}\right) \bar{n} z \pm i b z\right]$.

The elliptical normal modes are characterized by the following relations between their semi major and semi minor electric field amplitude components

$$
\left(\frac{E_{x}}{E_{y}}\right)_{ \pm}=i \frac{\alpha}{\frac{\beta}{2} \pm \sqrt{\alpha^{2}+\left(\frac{\beta^{2}}{4}\right)}}
$$

and

$$
\left(\frac{E_{x}}{E_{y}}\right)_{+}=\frac{1}{\left(E_{x} / E_{y}\right)_{-}}
$$

where $x$ and $y$ coordinates denote the normal mode axes of linear birefringence retardation. The rotation $\theta$ of the semi major axis of the polarization ellipse is given by

$$
\theta=\frac{1}{2} \tan ^{-1} \frac{2\left(\left|E_{x}\right|\left|E_{y}\right|\right)}{\left(\left|E_{x}\right|^{2}-\left|E_{y}\right|^{2}\right)} \cos \Delta \varphi
$$

Here $\cos \Delta \varphi$ is the phase difference between the $x$ and $y$ components of the polarization ellipse.

Elliptical birefringence occurs naturally in magneto-optic waveguides as a consequence of geometrical confinement and stress. This combined effect of linear birefringence and optical gyrotropy in magneto-optic PCs plays an important role in optical sensor performance and will be discussed in proceeding chapters. 


\subsection{Iron garnets}

Bismuth substituted iron garnets are among the most commonly used materials for magneto-optical application in optical integrated circuits due to their high Faraday rotation and low optical absorption in the near infrared optical wavelength region [2932].

Iron garnets have a cubic crystal structures corresponding to the Ia3d symmetry group with a formula unit of $\mathrm{C}_{3} \mathrm{~A}_{2} \mathrm{D}_{3} \mathrm{O}_{12}$, and 160 ions per unit cell. In the crystal structure, the ' $\mathrm{C}$ ' sites are dodecahedrally-coordinated and occupied by rare earth ions $(\mathrm{Gd}, \mathrm{Lu})$ or $\mathrm{Y}^{3+}, \mathrm{Bi}^{3+}$ cations, as shown in Fig. 2.9 for the case of yttrium iron garnet. Two tetrahedral sites (A) and three octahedral (D) sites are occupied by iron ions forming oppositely magnetized sub-lattices (See Fig. 2.9). The ions occupying the dodecahedral sites are either non magnetic with filled outer electron shells or weekly magnetic leaving iron be the main contributor for magnetic effects of the material.

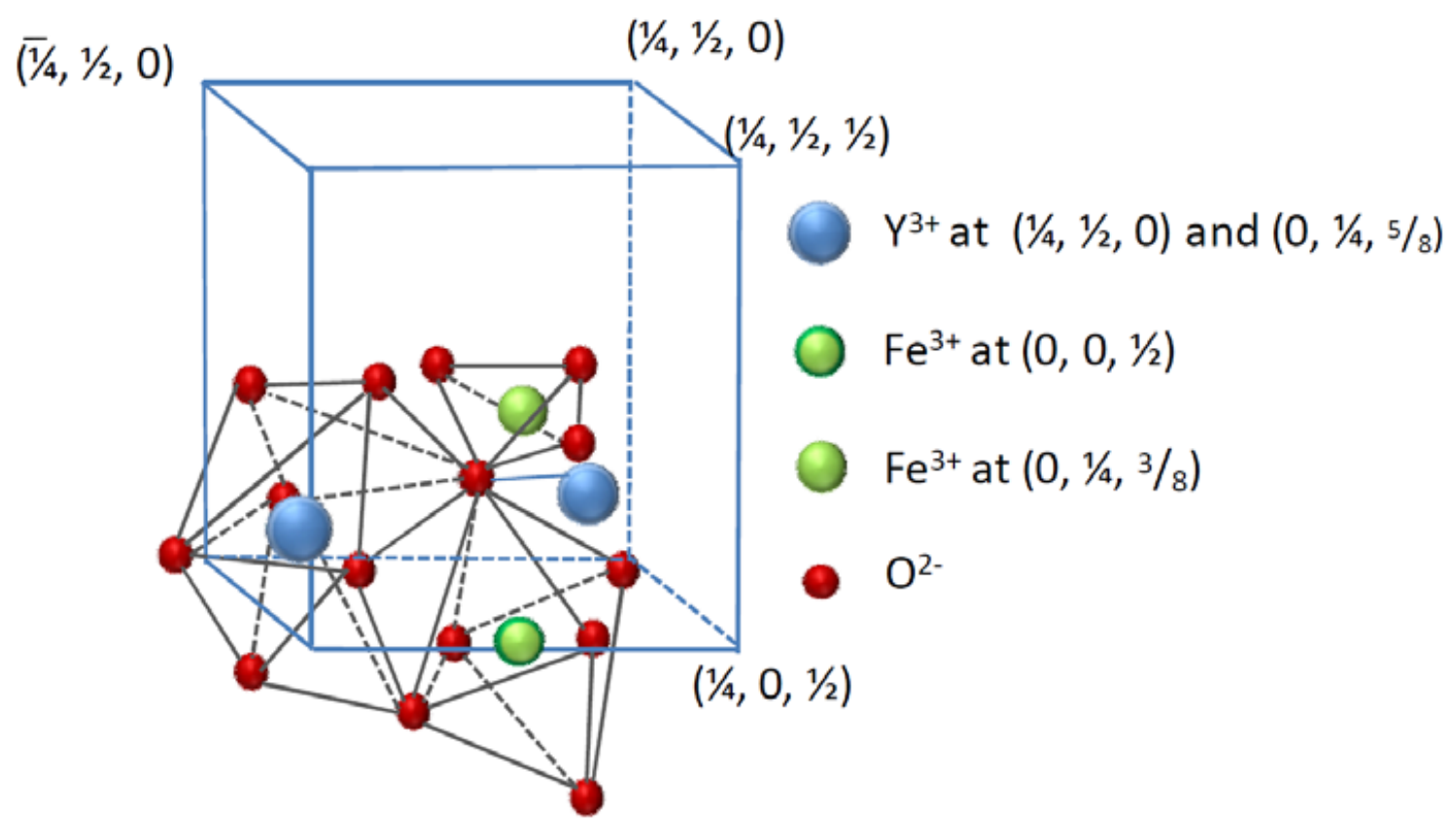

Figure 2.9 Lattice structure of yttrium iron garnet . (Courtesy: Zhuoyuan Wu PhD dissertation, permission details in appendix B) 
The ' $A$ ' and ' $D$ ' lattices couple anti-ferromagnetically resulting in a net magnetic moment. Hence the iron garnets are ferrimagnetic with a net magnetic moment.

The vector sum of the sublattice magnetizations determines the saturation magnetization $M s$ of the garnet crystal. $M s$ is a temperature-dependant quantity. With the assumption that the dodecahedral sub-lattice makes no contribution to the magnetic moment of the crystal, the saturation magnetization of the garnet crystal can be written as in Eq. 2.44 below.

$$
M_{s}(T)=M_{d}(T)-M_{a}(T)
$$

where $M_{a}$ and $M_{d}$ denote the saturation magnetizations of the octahedral and tetrahedral sub-lattices, respectively [33].

Magnetic garnet thin films can be grown by liquid-phase epitaxy (LPE), sputter deposition or pulsed laser deposition (PLD) techniques. Material properties of garnets can be tailored according to the application requirements by varying the chemical composition, including the rare-earth ion substitution levels, and growth parameters.

The Faraday rotation for a specific magnetic garnet material can vary with the temperature $T$, the optical wavelength $\lambda$, and on the crystal sub-lattice magnetizations.

\subsection{Photonic crystals}

Photonic crystals can be simply defined as a periodic distribution of refractive indices in the space domain. Even though modern scientific research utilizes artificially created photonic structures to manipulate light, there exist numerous natural photonic structures in nature. These natural photonic structures can be found in insects, birds, flora and fauna [34].

In order to manipulate light using photonic crystals, the order of the periodicity of the structures should be of the order of the wavelength of light. Periodic macroscopic stacks of dielectric materials in photonic crystals are analogous to the crystals lattice structures of semiconductor materials. The periodic potential against the propagation of electrons present in the semiconductor lattice crystal is replaced by the periodic 
dielectric constant in the photonic crystal acting against the propagation of photons. If the dielectric constant difference is strong enough the refraction and the reflection of light through the material can produce a band gap for some frequencies, similar to band gaps in semiconductors.

The first theoretical prediction of artificial photonic crystals was done by Yablonovitch and John in 1987 [35,36]. Since then, there have been numerous publications and development in the field of photonic research [37-45]. Photonic crystals can be designed to control of the desired frequencies by careful choice of materials and dimensions. For example, for microwave control of light could be achieved by photonic structures constructed in millimeter scale while infrared control of light could be achieved by PC structures constructed in micrometer scale $[37,42]$.

Depending on the periodic variation of the material arrangement, there could be one dimensional, two dimensional and three dimensional photonic crystals. Given a sufficient difference in periodic dielectric constant in the structure, a three dimensional photonic crystal could create a complete band gap for certain frequencies. Figure 2.10 shows a schematic representation of 1-D, 2-D and 3-D photonic crystal arrangements.
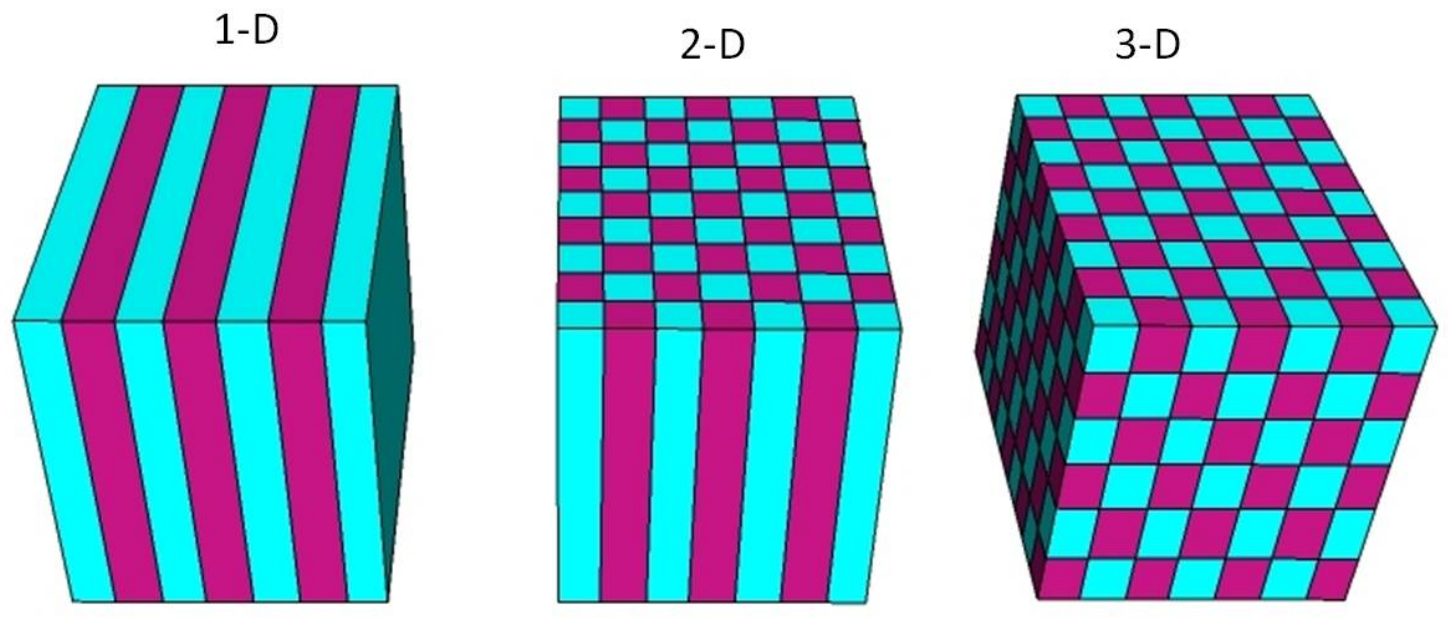

Figure 2.10 Periodic layer arrangement of simple 1-D, 2-D and 3-D photonic crystal structures 
While perfect photonic crystal structures create photonic band gaps, photonic crystals with line defects and point defects are used for important applications such as photonic crystal waveguides and micro cavity devices [46-51]. A line defects or a point defect can be introduced to the photonic crystal by breaking the spatial periodicity of the structure. Defects create localized modes within the photonic band gap which allows light to propagate only in the frequencies defined by the defect structure. In two dimensional case, a point defect can be created by simply removing a single column or by replacing it with another column with a different size, shape or dielectric constant. A photonic crystal waveguide can be designed by introducing a linear defect (removing a single row of columns) in to the two dimensional crystal [37,42]. Photonic crystal waveguides are highly efficient compared to conventional total internal reflections based waveguides when transmitting light at tight bends and narrow paths. Another application of photonic crystals and also relevant to this dissertation is bio/chemical sensing [1,5,7-9]. Mostly two dimensional PC structures with defects or cavities have been explored for this purpose. In this work we explore one dimensional magnetophotonic crystal structures for optical sensing.

\subsubsection{Photonic band gaps}

Photonic band gaps are the optical analogue of electronic band gaps in semiconductor crystals. The propagation of light in photonic crystals can be understood through solutions of the Maxwell's equations. In the absence of free charges and currents, for a homogeneous dielectric material, Maxwell's wave-equation can be written as follows,

$$
\left\{\nabla \times\left(\frac{1}{\varepsilon(r)}\right) \nabla \times\right\} \mathrm{H}(\mathrm{r})=\frac{\omega^{2}}{\mathrm{c}^{2}} \mathrm{H}(\mathrm{r})
$$

Here $\mathrm{H}(\mathrm{r})$ is the magnetic field of the photon, $\varepsilon(\mathrm{r})$ is the macroscopic dielectric permittivity, $\omega$ is the photon frequency and $c$ is the speed of light. The periodic variation in dielectric permittivity in a PC can be written $\varepsilon(r)=\varepsilon\left(r+R_{i}\right)$ where $R_{i}$ is the lattice vector with $i=1,2,3$ for three dimensional spatial variation. Given $\varepsilon(r), a$ 
periodic function following the Bloch-Floquet theorem, solutions to Eq. 2.45 are of the form $H(r)=e^{i k r} H_{n, k}(r)$ with eigenvalues $\omega_{n}(k) . \quad H_{n, k}(r)$ is a periodic envelope function satisfying

$$
(\nabla+i k) \times \frac{1}{\varepsilon}(\nabla+i k) \times H_{n, k}=\left(\frac{\omega_{n}(k)}{c}\right)^{2} H_{n, k}
$$

which yields discrete eigenvalues $\omega_{n}(k)$ with $\mathrm{n}=1,2 \ldots$ for a periodic structure. $\omega_{n}(k)$ are continuous functions of $k$, form discrete bands when plotted against $k$ in a dispersion diagram.

The eigensolutions $\omega_{n}(k)$ are also periodic functions of $k$ yielding that solutions at $k+G j$ are the same as at $k$ where $G j$ is the reciprocal lattice vector defined by $R_{i} . G_{j}=$ $2 \pi \delta_{i j}$. For a one-dimensional periodic structure with periodicity $a$, and $G_{I}=\frac{2 \pi}{a}$, the $k$ space between $\frac{\pi}{a}$ and $\frac{\pi}{a}$ is called the first Brillouin zone of the system.

A photonic band gap is a region where there is no propagation of light frequencies allowed. Figure 2.11(left) shows the dispersion relation $\omega(k)=c k$ for the propagation of electromagnetic waves in a one-dimensional homogenous medium. Bands for $|k|>\frac{\pi}{a}$ are folded back into the first Brillouin zone and shown by a dashed line. The wave solutions with electric fields $E \sim e^{ \pm \pi x / a}$ can be expressed as a linear combination of $e(x)=\cos (\pi x / a)$ and $o(x)=\sin (\pi x / a)$. In the presence of dielectric periodicity band gaps open up at $\pm \frac{\pi}{a}$ by degeneracy breaking of $e(x)$ and $o(x)$. The field $e(x)$ is more concentrated in the higher $\varepsilon\left(\mathrm{n}_{\text {high }}\right)$ regions and $o(x)$ is more concentrated in the lower $\varepsilon\left(\mathrm{n}_{\text {low }}\right)$ regions, forming the lower and upper edges of the band gap respectively. Therefore any variation in dielectric constant in one dimensional system will lead to a band gap [Fig. 2.11 (right)]. The strength of the band gap is determined by the strength of the dielectric constant variation [37-39, 42]. 


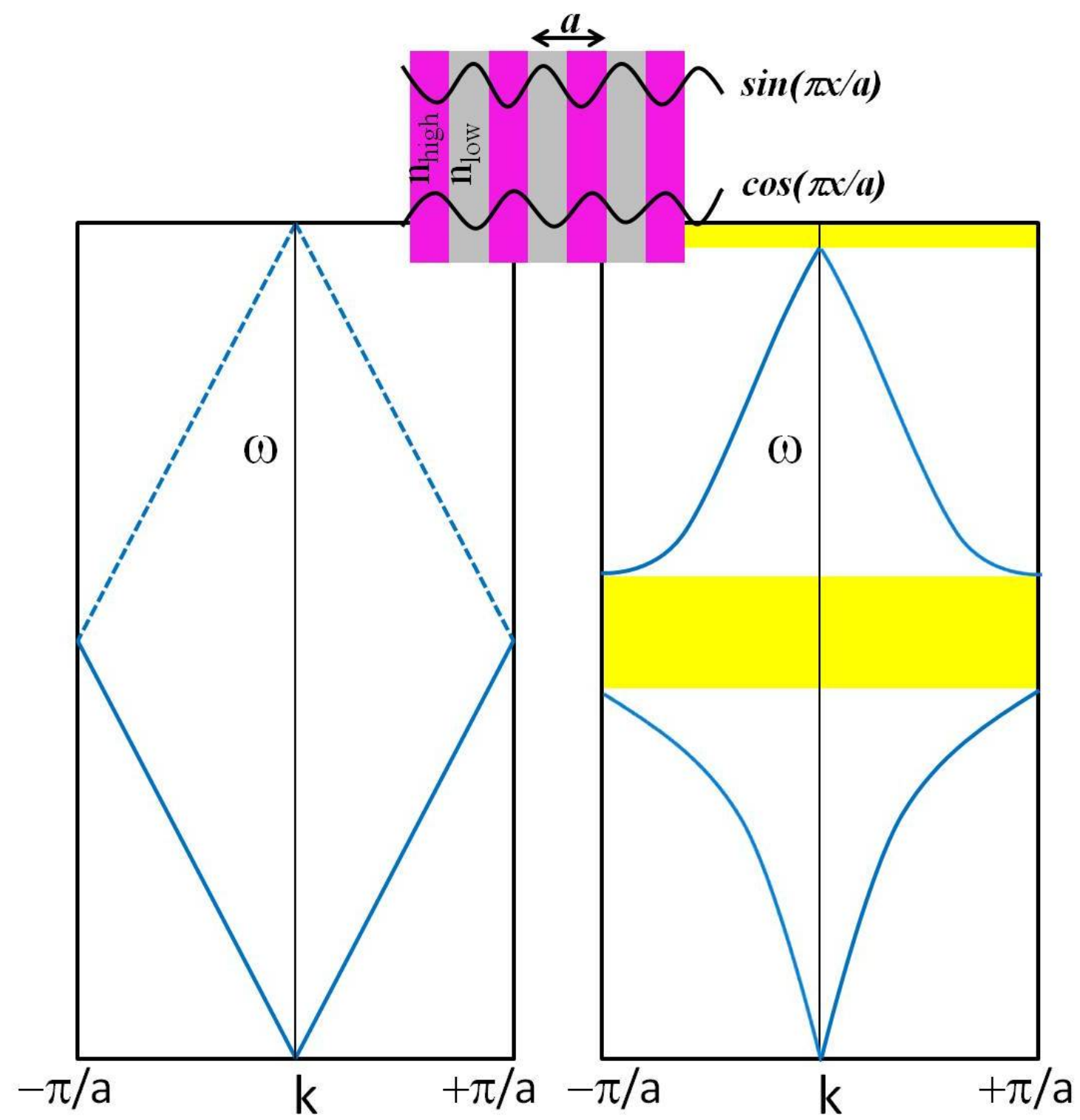

Figure 2.11 Left: Dispersion diagram of a homogeneous one-dimensional medium. Right: Band diagram of a one dimensional material system with a dielectric variation of period $a$. A gap is opened at the Brillouin zone boundary at $k= \pm \frac{\pi}{a}$. Inset: The dielectric periodicity in the system breaks plane waves of a uniform medium into $\cos (\pi x / a)$ and $\sin (\pi x / a)$ standing waves [38]. 


\subsubsection{Magneto photonic crystals}

For photonic crystals where the constituent materials are magnetic, the resultant PCs are known as magneto-photonic crystals (MPCs) [52]. Photonic crystals combined with magneto-optical effects exhibit very unique optical and magneto-optical properties. The combination of magnetic tunability, Faraday rotation, and bandgap engineering in MPC structures make them especially attractive for integrated optical applications. Enhanced Faraday rotation, fast optical switching and ultra small optical isolators are some of the well developed phenomena [28,29,52-67]. In this dissertation research, we investigate the polarization rotation of MPCs and their sensitivity to cover refractive index in the waveguide geometry.

Recent work by professor Levy's research group on one-dimensional Bragg waveguides in gyrotropic systems has revealed the presence of multiple band gaps arising from waveguide mode coupling and large polarization rotations near the photonic band gap edges [11-16, 28,63-72]. Polarization rotation enhancement due to photon trapping has also been reported by other research groups for one-dimensional layered stacks with gyrotropic resonant cavities $[29,58]$. The rotation enhancement is explained in terms of resonant enhancement in photonic path length leading to an increase in net Faraday rotation from multiple passes in a non-reciprocal medium. However, the situation in gyrotropic Bragg waveguides, even ones with resonant cavities or half-wave steps, is often fundamentally different because of the presence of linear birefringence. Linear birefringence tends to suppress the Faraday rotation and yet large magneto-optic polarization rotations, responsive to magnetic field reversals, have been reported by Professor Levy and co-workers even for significant linear birefringence in the high-order modes [11-16]. A detailed discussion on the rationale behind the high polarization rotations in these systems are given in sections 4.3 and 4.4. 


\subsubsection{One dimensional Bragg waveguides}

Bragg waveguides consist of a 1-D grating fabricated into a waveguide structure where grating dimensions are selected to satisfy the Bragg condition. This method allows the formation of a 1-D photonic crystal in a planar geometry. In this work the 1-D grating structure is patterned onto a multimode ridge waveguide by focused ion beam (FIB) milling. Figure 2.12 shows a schematic depiction of the grating structure on a ridge waveguide and the scanning electron micrograph (SEM) of a grating fabricated by FIB milling.

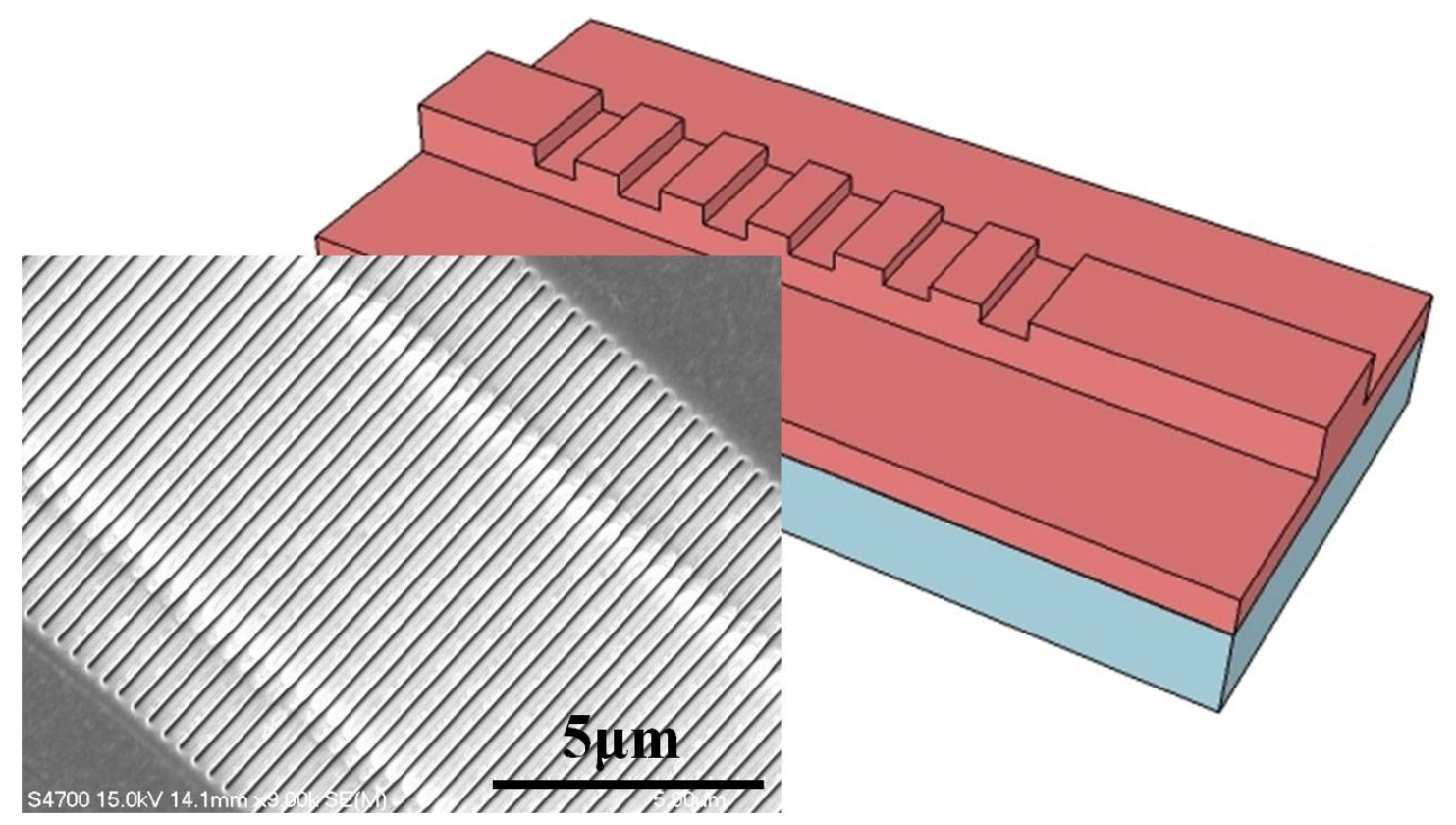

Figure 2.12 Schematic depiction of the Bragg grating on magneto-optic ridge waveguide with SEM micrograph of the grating region fabricated by FIB milling

Multiple bands gaps appear in the transmittance spectrum of such gratings. The band gap formation mechanism can be understood by the phase matching condition of the optical waves by grating. Depending on the thickness of the waveguide, a ridge waveguide can support more than one guided modes. The contra directional coupling 
(shown in fig. 2.13) of the guided modes is responsible for formation of band gaps in the transmittance spectrum [20].

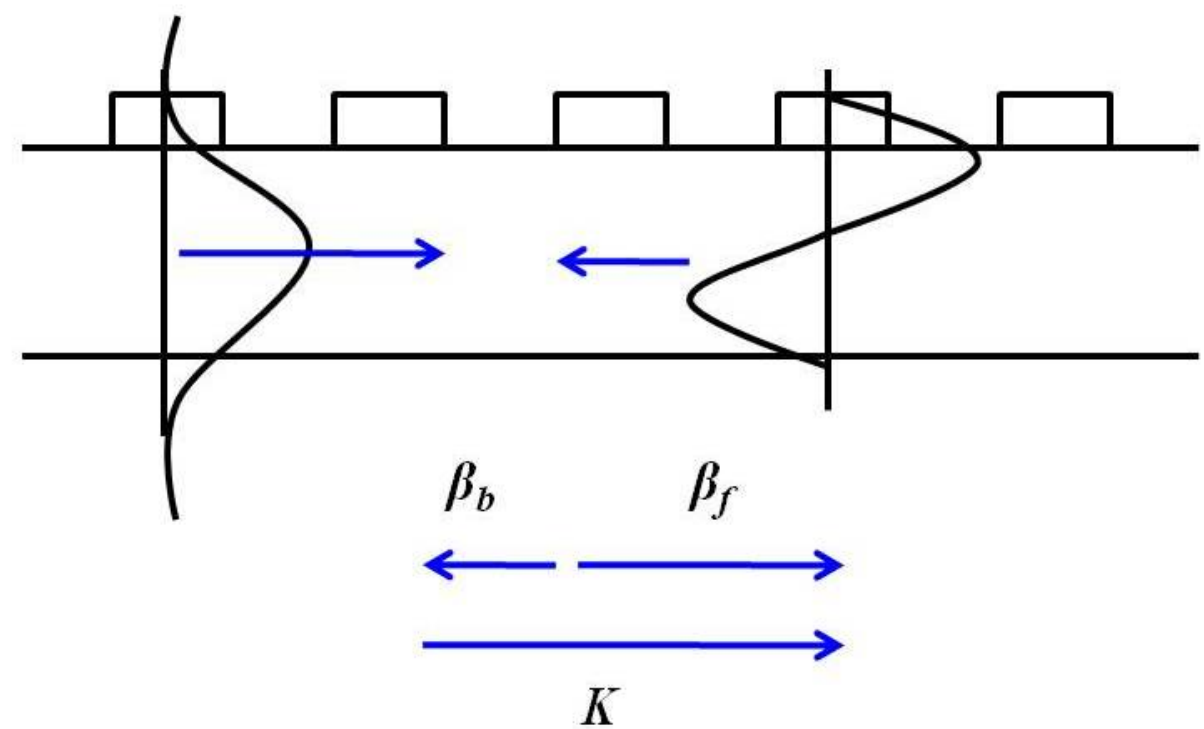

Figure 2.13 Contra directional coupling of waveguide modes by a grating.

The space matching condition for coupling between two optical waves given by,

$$
\beta_{b}=\beta_{f}+q K, \quad q=0, \pm 1, \pm 2 \ldots
$$

Where $\beta_{b}, \beta_{f}$ are propagation constants of the forward and backward propagating waveguide modes. $K=\frac{2 \pi}{\Lambda}$ and $\Lambda$ is the grating period. The 3-D relation given by expression 2.47 is the well known Bragg condition for photonics [20]. Equation 2.47 can be rewritten with substituting propagation constants with their subsequent effective indices, 


$$
\left(n_{f}+n_{b}\right) \Lambda=\lambda
$$

where $\lambda$ is the optical wavelength in the medium [11]. Therefore, the multiple band gaps observed in magneto-optic Bragg gratings can be accounted for the coupling between forward fundamental waveguide to reflected fundamental and higher order waveguide modes.

The presence of Bragg gratings induces the formation of Bloch states in the periodic structure. These Bloch modes are generally elliptically polarized in birefringent gyrotropic media with non-negligible birefringence. However their polarization state and frequency dispersion characteristics differ from those of the elliptical normal modes that propagate in regions of uniform thickness outside the grating.

\subsection{Wave propagation in birefringent magneto-optic media}

Magnetic birefringent media magnetized along the direction of propagation break linearly polarized light in to right and left circularly polarized light. These counterrotating normal modes propagate with different speeds in the media. At the presence of both linear and magnetic circular birefringence, the normal modes are no longer circularly polarized but elliptically polarized states.

The relative permittivity tensor $\varepsilon$ of the medium magnetized along the $z$ direction given by,

$$
\varepsilon=\left(\begin{array}{ccc}
\varepsilon_{x x} & i \varepsilon_{x y} & 0 \\
-i \varepsilon_{x y} & \varepsilon_{y y} & 0 \\
0 & 0 & \varepsilon_{z z}
\end{array}\right)
$$

where we assume no absorption of the light in the medium which implies that all components of the relative permittivity $\left(\varepsilon_{i j} i, j=x, y, z\right)$ are real.

The four Maxwell equations in the absence of charge and current sources are

$$
\nabla . D=0
$$




$$
\begin{gathered}
\nabla \cdot B=0 \\
\nabla \times E=-\frac{\partial B}{\partial t} \\
\nabla \times H=\frac{\partial D}{\partial t}
\end{gathered}
$$

The wave equation is given by,

$$
\nabla \times \nabla \times E-\frac{\omega^{2}}{c^{2}} \varepsilon E=0
$$

Hence a plane wave with electric field $E=E_{0} e^{i(k \cdot r-\omega t)}$ in birefringent magneto-optic media can be written as,

$$
\left(k_{0}^{2} \varepsilon-k^{2} I+k k\right) \cdot E_{0}=0
$$

Where $k_{0}=\frac{\omega}{c}$, c is the speed of light in vacuum. $E_{0}$ is the plane-wave amplitude, $I$ is the identity matrix. By solving the wave equation at normal incidence one can obtain the eiganvectors given by

$$
\hat{e}_{ \pm}=\frac{1}{\sqrt{2}}\left(\begin{array}{c}
\cos \alpha+\sin \alpha \\
\pm i \cos \alpha-\sin \alpha \\
0
\end{array}\right)
$$

With refractive indices $n_{ \pm}$, and $n_{ \pm}^{2}=\bar{\varepsilon} \pm \sqrt{\Delta^{2}+\varepsilon_{x y}^{2}}$, respectively. Here $\bar{\varepsilon}=\frac{\varepsilon_{y y}+\varepsilon_{x x}}{2}$ $, \Delta=\frac{\varepsilon_{y y}-\varepsilon_{x x}}{2}, \alpha=\frac{\gamma}{2}$ and $\gamma$ is defined as

$$
\begin{gathered}
\sin \gamma=\frac{\Delta}{\sqrt{\Delta^{2}+\varepsilon_{x y}^{2}}} \\
\cos \gamma=\frac{\varepsilon_{x y}}{\sqrt{\Delta^{2}+\varepsilon_{x y}^{2}}}
\end{gathered}
$$


Therefore input linearly polarized light become elliptically polarized with rotated plane of polarization with respect to the incident light upon propagating through such media.[14,69,72]

\subsection{Gyrotropic bandgaps in elliptically birefringent media}

The propagation of electromagnetic waves in an elliptically birefringent magneto-optic layered system leads to the formation of new type of band gaps inside the Brillouin zone. This new type of band gap stems from coupling between the different elliptical polarization states in anisotropic magneto-photonic systems. In planer waveguide MPCs this is achieved by coupling between different counter-propagating waveguide modes.

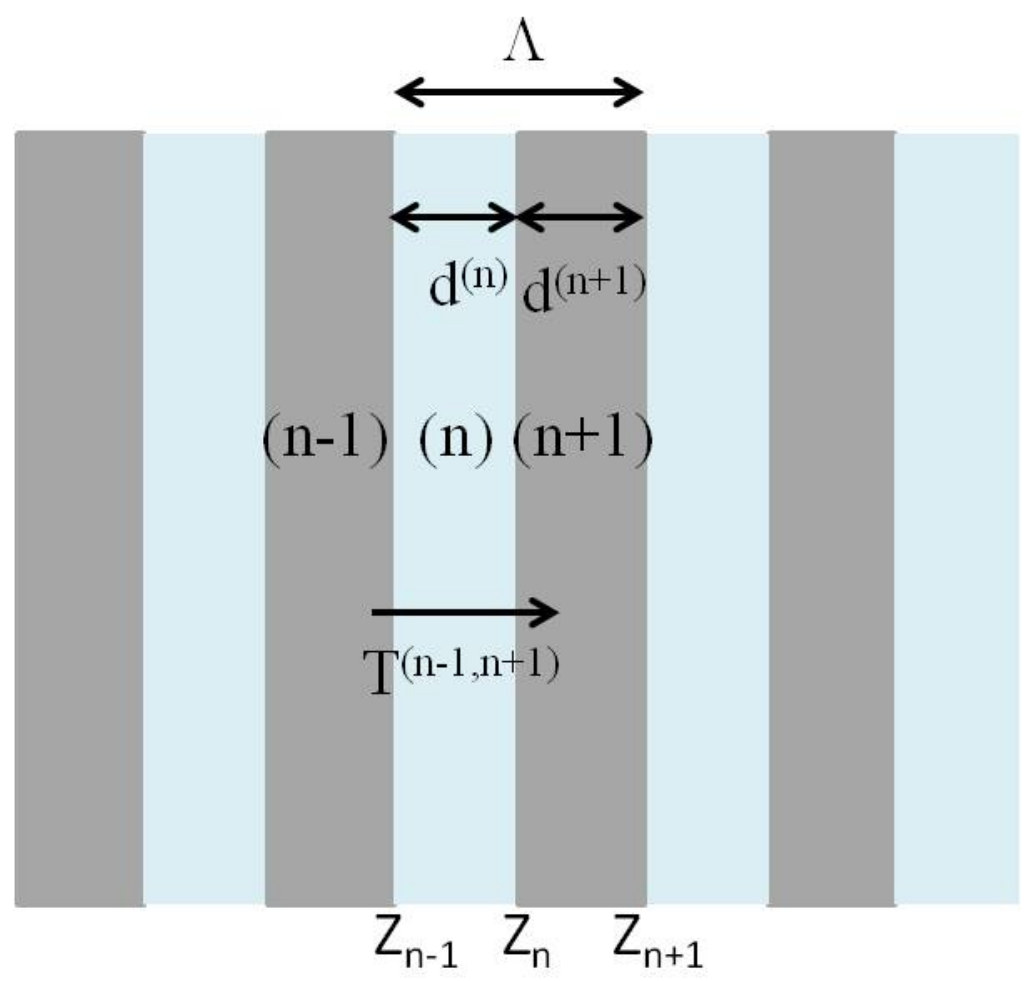

Figure 2.14 Schematic diagram of a one-dimensional birefringent magnetophotonic crystal with period of $\Lambda$. The structure extends indefinitely in the $x$ and $y$ directions. A unit cell is given by the region between $z_{n-1}$ and $z_{n+1}$. [13] 
M Levy et al have developed a theoretical stacked layer (Fig. 2.14) model with different elliptically birefringent normal modes in alternating layers which mimics onedimensional PC in waveguide configuration [12-14]. The model explains the formation of gyrotropic band gaps as a result of the coupling between counter-propagating Bloch modes and allows one to design the spectral response of the system and the bandwidth of the gyrotropic band gap.

The Bloch states for the system are expressed as a linear combination of these local normal modes, subject to the Floquet-Bloch theorem. Forward- and backward-traveling local modes are allowed to differ in refractive index and elliptical-polarization state to account for the contra-directional coupling between modes of different order in the waveguide. The Block mode in layer $n$ is expressed as follows,

$$
\begin{aligned}
& E(z, t)= \\
& {\left[\left(E_{01} \exp \left(i \frac{\omega}{c} n_{+}^{f}\left(z-z_{n}\right)\right)\right) \hat{e}_{+}^{f}+\left(E_{02} \exp \left(-i \frac{\omega}{c} n_{+}^{b}\left(z-z_{n}\right)\right)\right) \hat{e}_{+}^{b}\right] \exp (-i \omega t)+} \\
& {\left[\left(E_{03} \exp \left(i \frac{\omega}{c} n_{-}^{f}\left(z-z_{n}\right)\right)\right) \hat{e}_{-}^{f}+\left(E_{04} \exp \left(-i \frac{\omega}{c} n_{-}^{b}\left(z-z_{n}\right)\right)\right) \hat{e}_{-}^{b}\right] \exp (-i \omega t)}
\end{aligned}
$$

Here superscripts $f$ and $b$ refer to the forward and backward propagating modes. The refractive indices $n_{ \pm}^{f, b}$ correspond to modes of opposite helicity. The elliptical polarization-state unit-vectors $\hat{e}_{ \pm}^{f, b}$ are given by

$$
\hat{e}_{ \pm}^{f, b}=\frac{1}{\sqrt{2}}\left(\begin{array}{c}
\cos \alpha^{f, b} \pm \sin \alpha^{f, b} \\
\pm i \cos \alpha^{f, b}-i \sin \alpha^{f, b} \\
0
\end{array}\right)
$$


The Bloch states for this system are given through solutions to following eigenvalue equation. The solutions satisfy Floquet-Bloch theorem.

$$
T^{(n-1, n+1)} E=\exp (i K \Lambda) E
$$

The transfer matrix $T^{(n-1, n+1)}$ transforms the Bloch state by one unit cell. Here $K$ is the Bloch wave vector and $\Lambda$ is the period of the periodic structure.

The mode coupling depends on the relative elliptical birefringence of the adjacent layer normal modes and their individual propagation constants. If there is no relative elliptical birefringence of the normal modes of the adjacent layers, the modes remains uncoupled. At the presence of non-zero relative elliptical birefringence, the adjacent layer normal modes are coupled satisfying the boundary conditions. Hence, the gyrotropy of the system in combination with linear birefringence leads the normal modes of adjacent layers acquire different elliptical polarizations which in turn leads to the mode coupling and formation of the new band gap. Considering the role of gyrotropy in existence of this bands gap, it is denoted as gyrotropic bandgaps $[12,13]$. The model predicts slightly shifted band gaps (band gap "doublets") form inside the Brillouin zone and enhanced polarization rotation due to partial suppression of Bloch modes at the band edges for 1-D birefringent MPCs. Another important prediction of the model is that the polarization rotation is very sensitive to variations in refractive index or linear birefringence in the film, substrate, or cover layer [14]. This has been the basis for the optical sensor developed in this dissertation research.

\subsection{Conducting polymers for chemical sensing}

The conducting polymers such as polyaniline, polypyrrole and polythiophene attract attention of researchers from a variety of fields in science and technology as promising electrode materials for energy storage devices, electrocatalysts and biosensors $[3,10,73,74]$. Conducting polymers in their pure form posses rather low conductivities $\left(<10^{-5} \mathrm{~S} \mathrm{~cm}-1\right)$ [76]. The process of doping could produce significant increase in the 
conductivities. The doping is performed by means of chemical or electro-chemical oxidation (p-doping) or reduction (n-doping). In general, sensing mechanisms of conducting polymer based sensors are directly linked to the doping and undoping of the polymer $[3,10]$. The use of conducting polymers and their derivatives as the active layers of gas/chemical sensors dates back to early 1980s. Conducting polymer based sensors demonstrate high sensitivities and short response time and most importantly they are operational at room temperature $[10,73,74]$.

\subsubsection{Polypyrrole}

Polypyrrole, Polyaniline and Polypthiophene are some of the conducting polymers which have been researched for chemical sensing. However, PPy attracted special attention because of the high conductivity, environment stability and good mechanical properties combined with their ease and high flexibility in preparation [73-79]. PPy films can be used for the detection of many analytes such as ammonia, nitrogen oxides, carbon monoxide, sulphur dioxide, hydrogen sulphide and methanol[73]. Polypyrrole is synthesized from the pyrrole monomer (Fig.2.15) by mild oxidation, using chemical or electrochemical techniques. Electrochemical method has a good control over the oxidation reaction. Chemical synthesis is relatively simple and the oxidation reaction can be controlled by the process parameters such as concentration of monomer, dapant, oxidant, process temperature and deposition time. In addition chemical synthesis is inexpensive, hence suitable for mass production [74-79]. Aqueous or anhydrous $\mathrm{FeCl}_{3}$, other salts of iron (III) and copper (II) are widely used as chemical oxidants. However research shows $\mathrm{FeCl}_{3}$ is the most suitable oxidant for chemical polymerization of PPy. Monomer oxidation preparation of $\mathrm{PPy}$ with $\mathrm{FeCl} 3$ in various solvents (water, alcohols, benzene, chloroform, acetone, acetonitrile) yields variation in the electrical conductivity (hence refractive index) in the end product. 
(a)

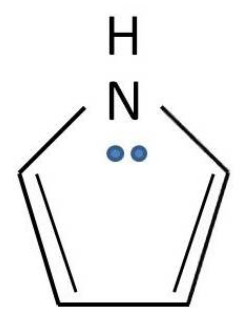

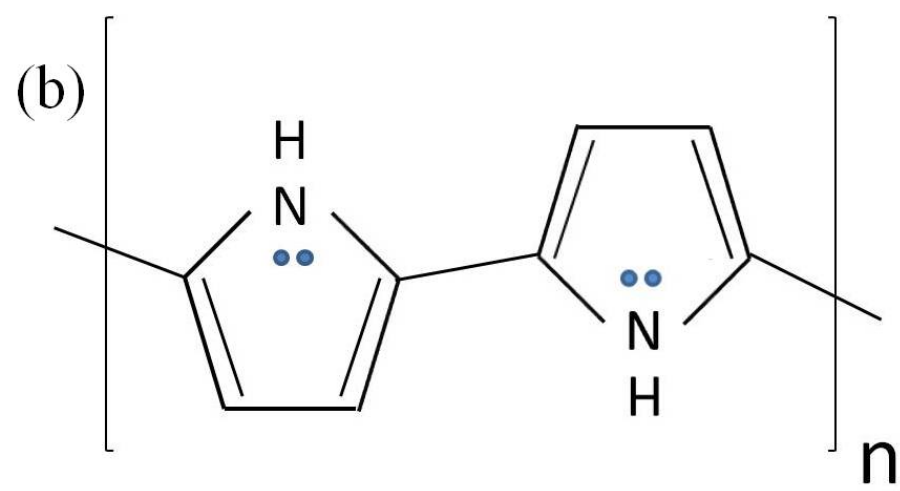

(c)

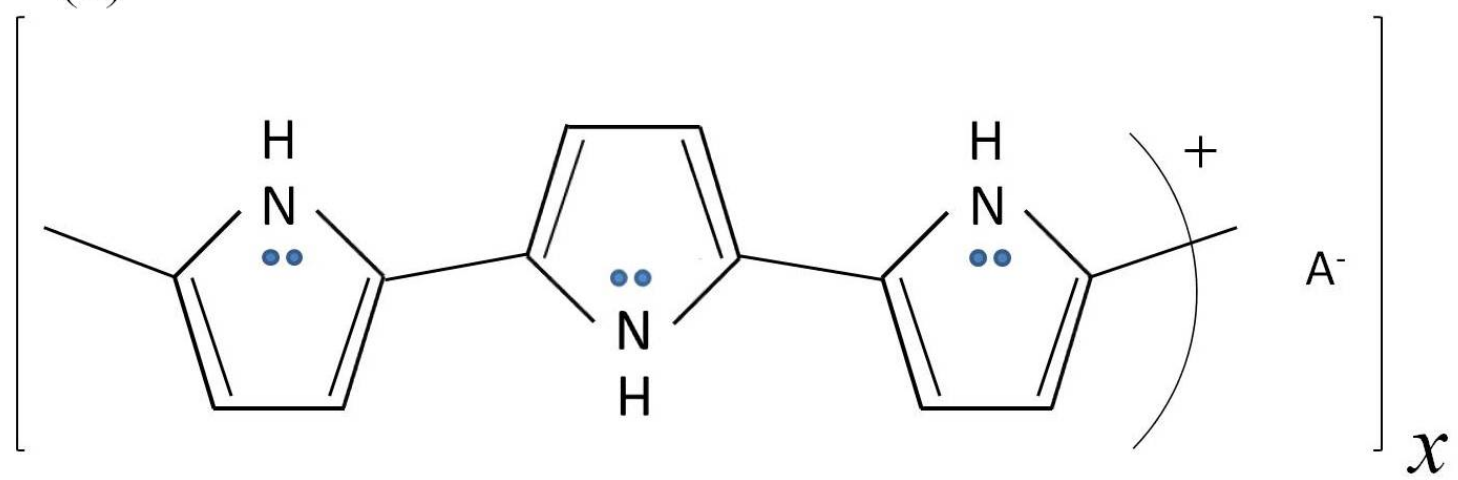

Figure 2.15(a) Pyrrole monomer, (b) neutral Polypyrrole, (c) oxidized Polypyrrole.

The stability in air of the doped PPy films is relatively high; their degradation occurs only above $150-300{ }^{\circ} \mathrm{C}$ and depends on the dopant anion. In reduced or undoped form, PPy tend to be less stable compared to doped PPy. Differences in synthesis methods, parameters and after treatments produce variations in the electrical, mechanical and morphological properties in Polypyrrole films [75-76].

\subsubsection{Sensing mechanism}

The interaction of gaseous components with deposited PPy films produces physical property changes in the film. There is a strong correlation between the physical properties of conducting polymers and their doping levels. The chemical reactions 
involved in doping and undoping of the conducting polymers are mostly redox reactions which transfer electrons between the polymer and the analytes. Electron transferring can cause the changes in conductivity hence refractive index of the sensing material. Interaction of oxidized PPy with electron acceptors such as $\mathrm{NO}_{2}$ and $\mathrm{I}_{2}$ leads to an increase in the doping level as well as the electric conductance of the conducting polymer. An opposite process will take place at interaction with an electron-donating analyte. Present study investigates the detection of aqueous Ammonia and methanol where both analytes are electron-donors. A decrease in conductivity of PPy on exposure to $\mathrm{NH}_{3}$ suggests activation of a de-doping process under ammonia atmosphere. The possible interaction of PPy and $\mathrm{NH}_{3}$ can be expressed as follows,

$$
\begin{array}{ll}
\mathrm{PPy}^{+}+\mathrm{NH}_{3} \rightarrow \mathrm{PPy}^{0}+\mathrm{NH}_{3}{ }^{+} & \text {Adsorption } \\
\mathrm{PPy}^{+}+\mathrm{NH}_{3} \rightarrow \mathrm{PPy}^{0}+\mathrm{NH}_{3}{ }^{+} & \text {Desorption }
\end{array}
$$

The research findings on interaction of ammonia with PPy show the occurrence of both reversible and irreversible interactions. The reversibility of the interaction depends on the concentration of ammonia and the duration of the exposure. If PPy is exposed to low concentrated ammonia for a short period of time, the reaction is reversible. An irreversible reaction takes place when PPy is exposed to higher concentration of ammonia for a prolonged period of time [10]. 


\section{EXPERIMENTS}

\subsection{Iron garnet thin film fabrication}

There are several methods for fabrication of iron garnet thin films. Among these methods liquid phase epitaxy, pulsed laser deposition and magnetron sputtering techniques are most frequently used. All the garnet materials used in this work have been fabricated by liquid phase epitaxy.

\subsubsection{Liquid phase epitaxy technique}

The liquid-phase-epitaxy film growth technique is one of the mostly employed thin film fabrication techniques, which provides homogenous and good crystalline quality films for modern optical and micro-electronic device applications [80-83]. This technique allows the fabrication of thin films of compound materials with desired optical and electronic properties. In the LPE film growth method, the films are grown on top of a crystalline substrate and lattice-matching of the material to be grown with the substrate is important to achieve high-quality films.

Single crystal gadolinium gallium garnet $\left(\mathrm{GGG} ; \mathrm{Gd}_{3} \mathrm{Ga}_{5} \mathrm{O}_{12}\right.$, lattice constant $=12.383 \mathrm{~A}^{\circ}$ ) is a commonly used substrate for magnetic garnet preparation since it has a close lattice match with rare earth substituted iron garnets [31,81]. A GGG substrate is dipped into a supersaturated melt of the material to be grown with an appropriate rotation speed and the garnet film is crystallized on the substrate. The film quality is greatly dependent on the substrate and the growth parameters. Garnet films which are required to have planar magnetization are grown on (100) GGG substrates [83]. 


\subsubsection{Magnetron sputtering}

Sputter deposition is a physical vapor deposition technique for depositing thin films. In sputtering systems, a target (cathode) and a substrate (anode) are placed in a vacuum chamber facing each other and gas ions are accelerated towards the cathode by the electric field between the cathode and the anode. Argon is often selected as the sputtering gas. Accelerated $\mathrm{Ar}^{+}$impinges on the cathode causing target atoms to be ejected from the cathode. At the same time, secondary electrons are produced which ionize more $\mathrm{Ar}$ atoms in the gas. The ejected target atoms are deposited on the substrate to form the thin film. In magnetron sputtering, a permanent magnet is placed behind or around the target. The magnetic field provided by the magnet confines the secondary electrons near the target. This considerably increases the ionization of the sputtering gas in the vicinity of the target, which helps to ignite the plasma at a lower pressure. A higher rate of ionization increases the sputtering yield and due to lower pressure, there are fewer collisions for target atoms which reach the substrate.

The sputtering parameters, the chamber pressure at sputtering and the targetsubstrate distance can greatly influence the quality and crystallinity of the sputtered film. Other parameters which can affect the film quality are the substrate temperature and the deposition angle. The stoichiometry of the sputtered film may differ from that of the target material $[80,84]$.

\subsection{Thin film characterization}

The magnetic garnet films used in this study are grown by the liquid phase epitaxy method on gadolinium gallium garnet substrates. These films are characterized with a Metricon prism coupler equipped with $\mathrm{He}-\mathrm{Ne}(632.8 \mathrm{~nm})$ and infrared $(1543 \mathrm{~nm})$ lasers. The schematic representation of a prism coupler set up and a typical screen shot are shown in Figs. 3.1 and 3.2, respectively. At certain angles of incidence, light couples into the film slab through the prism. 


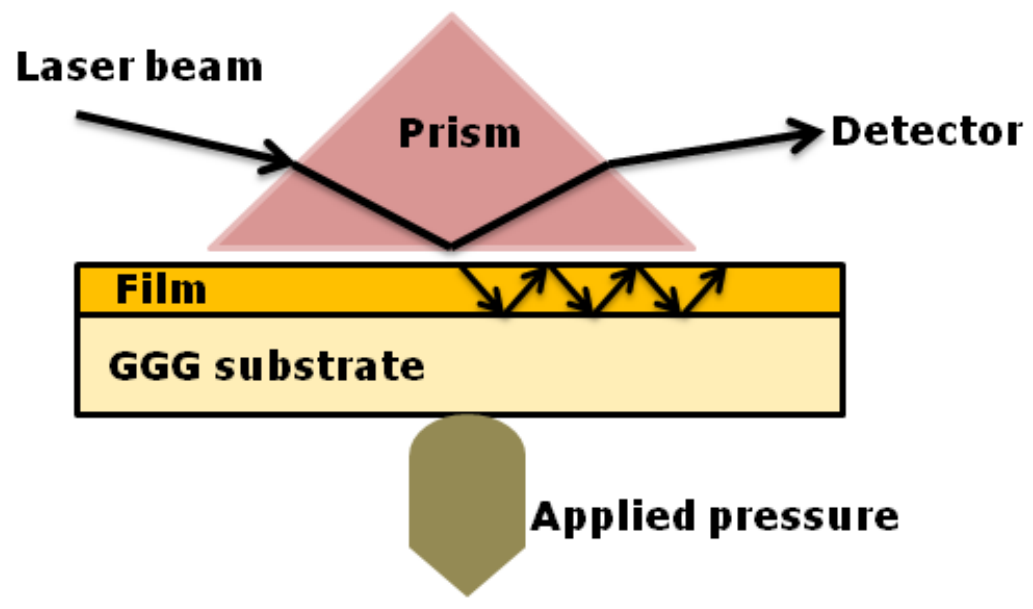

Figure 3.1 Schematic representation of the prism coupler set up.

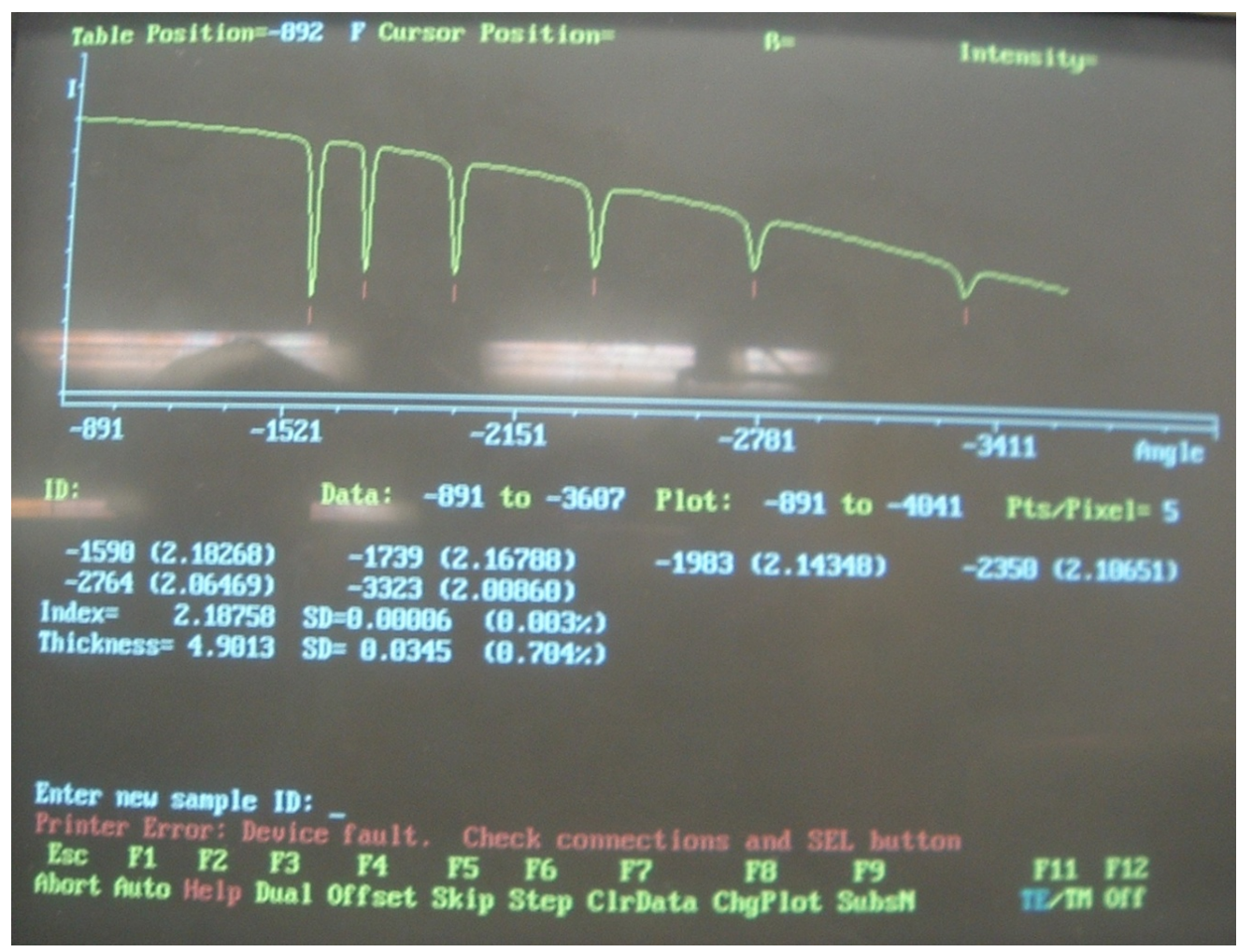

Figure 3.2 A screen shot of the prism coupler results for a $5 \mu \mathrm{m}$ thick film showing several guided modes for input TE polarization. 
The detector attached to the setup detects this drop in the intensity of light and records the relevant position in the output transmittance plot. Thus the film thickness, refractive index and waveguide mode indices of the TE and TM inputs of the film slab can be obtained using the prism coupler. Table 3-1 shows the thickness and the index measurements for TE and TM mode input polarization for a garnet film with the composition $\mathrm{Bi}_{0.8} \mathrm{Gd}_{0.2} \mathrm{Lu}_{2.0} \mathrm{Fe}_{5} \mathrm{O}_{12}$.

Table 3-1. Prism coupler measurements of wave guiding in the thin film slab for TE and TM input polarizations

\begin{tabular}{|c|c|c|c|c|c|}
\hline & Film index & $\begin{array}{c}\text { Fundamental } \\
\text { mode }\end{array}$ & $\begin{array}{c}1^{\text {st }} \text { order } \\
\text { mode }\end{array}$ & $\begin{array}{c}2^{\text {nd }} \text { order } \\
\text { mode }\end{array}$ & $\begin{array}{c}3^{\text {rd }} \text { order } \\
\text { mode }\end{array}$ \\
\hline $\begin{array}{c}\text { TE } \\
\text { polarization }\end{array}$ & 2.3182 & 2.30411 & 2.2616 & 2.1896 & 2.08661 \\
\hline $\begin{array}{c}\text { TM } \\
\text { polarization }\end{array}$ & 2.3192 & 2.30362 & 2.25627 & 2.17659 & 2.06505 \\
\hline Birefringence & -0.0010 & 0.00049 & 0.00533 & 0.01301 & 0.02156 \\
\hline
\end{tabular}

\subsection{Fabrication of waveguides}

Ridge waveguide fabrication involves several steps. The process is initiated with the optical characterization of the thin film followed by micro fabrication to obtain ridge waveguide structures onto the magnetic garnet thin films. The waveguide dimensions for efficient guiding of light are determined by beam propagation waveguide simulations [85].

\subsubsection{Waveguide dimension optimization}

Finite difference time-domain (FDTD) beam propagation simulations were utilized to optimize the waveguide dimensions, specifically waveguide width and ridge height for superior forward fundamental mode transmittance. 


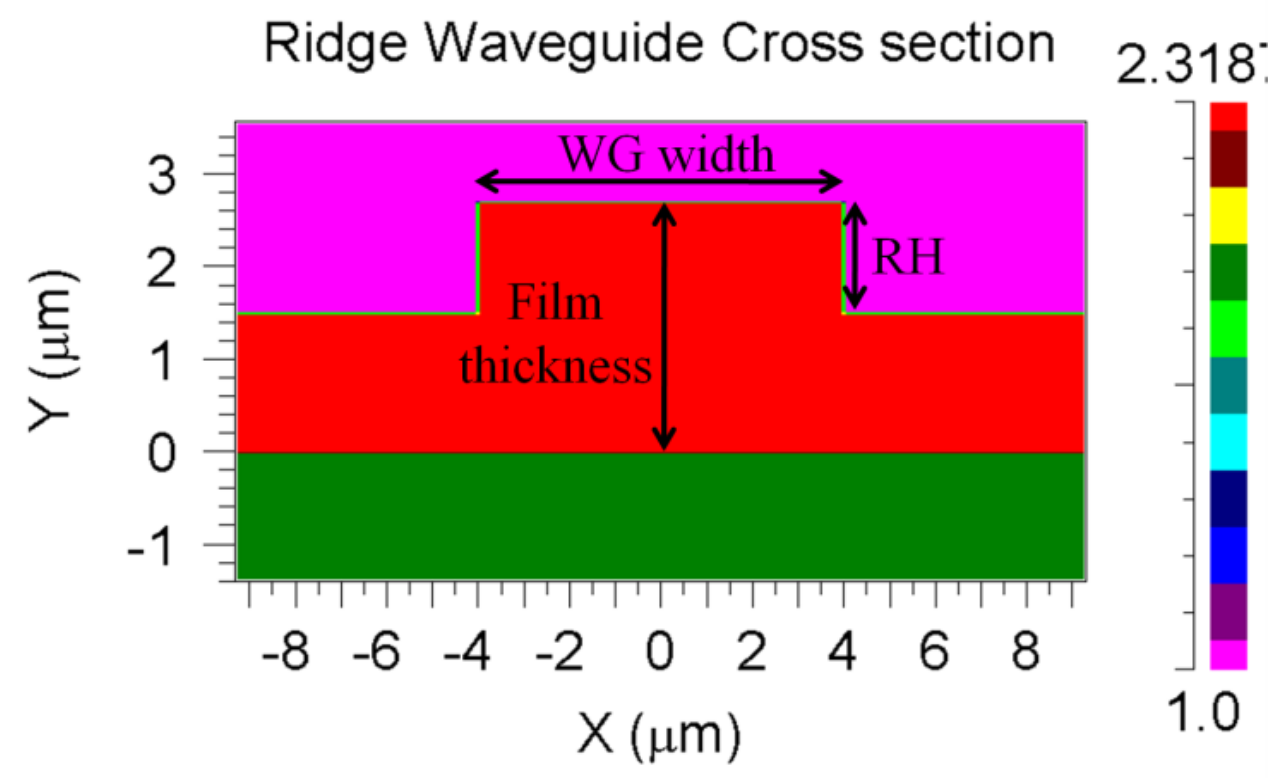

Figure 3.3 Ridge waveguide $x-y$ cross section

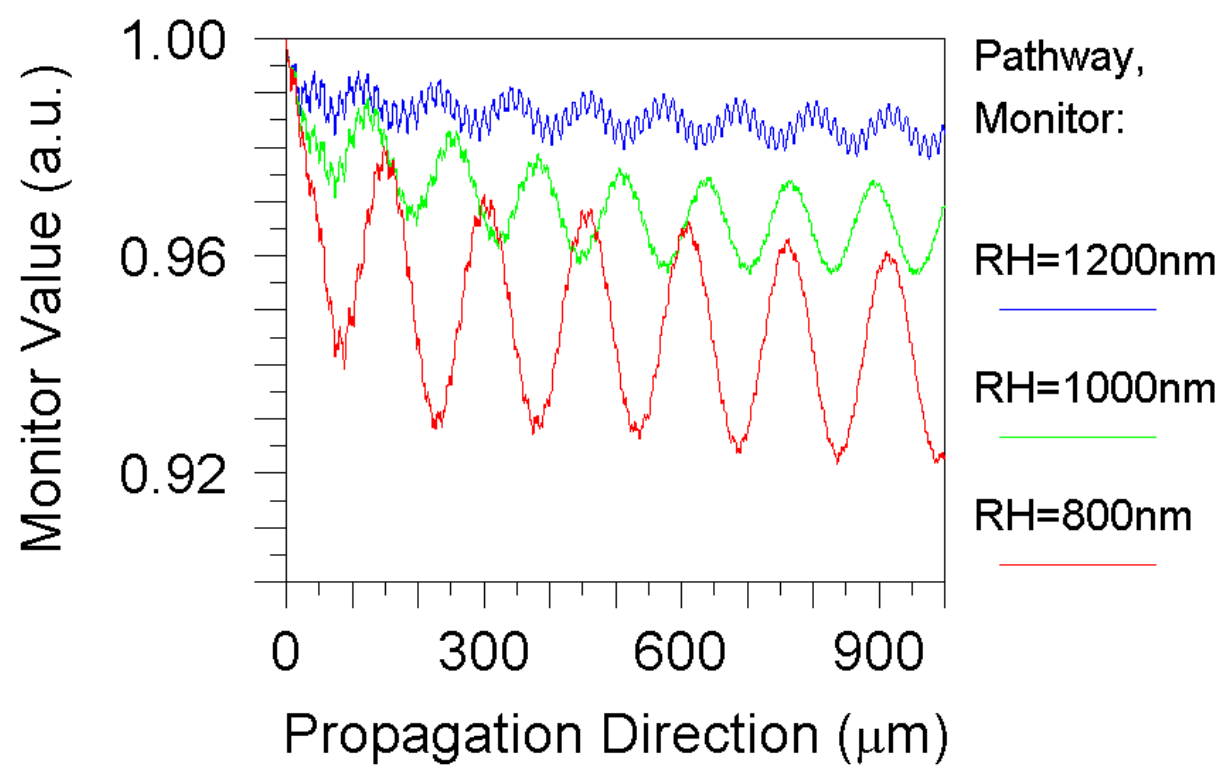

Figure 3.4 Transmitted power of a ridge waveguide designed on a film with thickness $2,71 \mu \mathrm{m}$ and refractive index 2.31. Transmitted power along the waveguide varies with the ridge height of the waveguide. 
For a $2.71 \mu \mathrm{m}$ thick film, a waveguide having a width of $8 \mu \mathrm{m}$ and ridge height $(\mathrm{RH})$ of $0.12 \mu \mathrm{m}$ delivers $98 \%$ of the coupled light in the forward transmittance. Figure 3.3 and Fig. 3.4 show the ridge waveguide cross section and the transmitted power (transmittance) along the waveguide for three different waveguide ridge heights respectively.

\subsubsection{Optical lithography}

Standard UV lithography is utilized to transfer waveguide patterns onto the iron garnet thin films from a pre-designed mask. Typical steps of the lithography involves sample cleaning, photo resist spinning, soft bake, UV light exposure in EVG 620 aligner, photoresist developing and hard bake.

The samples are first cleaned with acetone to remove any organic impurities. They are then cleaned with iso-propanol and de-ionized water. The cleaned samples are dried with $\mathrm{N}_{2}$ gas before they get air dried. A thin film of HMDS (Hexamethyldisilazane) is cast on the garnet sample by spinning with 3000RPM for a one minute in a photo resist spinner. HMDS enhances the adhesion of the photoresist onto the sample. Next, positive photoresist PR1827 is spun on the sample with 3000RPM for one minute to obtain a $2-3 \mu \mathrm{m}$ thick photoresist layer on the sample. The sample is soft baked for a one minute at $100^{\circ} \mathrm{C}$ to dry the photoresist layer to prevent it from adhering to the mask. The waveguide pattern on a chromium mask is then transferred onto the garnet sample by exposing the photoresist layer to UV radiation in EVG 620 aligner, at an aligner lamp intensity of $(\mathrm{I})=7.95 \mathrm{~mW} / \mathrm{cm} 2$ at $350 \mathrm{~W}$ power with a wavelength ranging from 240nm-350nm. The transferred pattern is developed in the photoresist developer MF319. This development process is carried out by gently shaking the sample, holding it vertically along the waveguide direction for about one minute. Subsequently, the developed sample is then cleaned with DI water to wash out the developer in order to prevent any overdeveloping. The sample with the transferred waveguide pattern is then hard baked on a hot plate at $100^{\circ} \mathrm{C}$ for 20 minutes to harden the photoresist. Figure 3.5 
shows a transferred waveguide pattern on a magnetic garnet thin film. The waveguide width is $6 \mu \mathrm{m}$.

The waveguide pattern obtained via optical lithography is processed through argon ion plasma etching in order to obtain the ridge waveguides. Ridge heights can be controlled as necessary via etching time with previously determined etch rate.

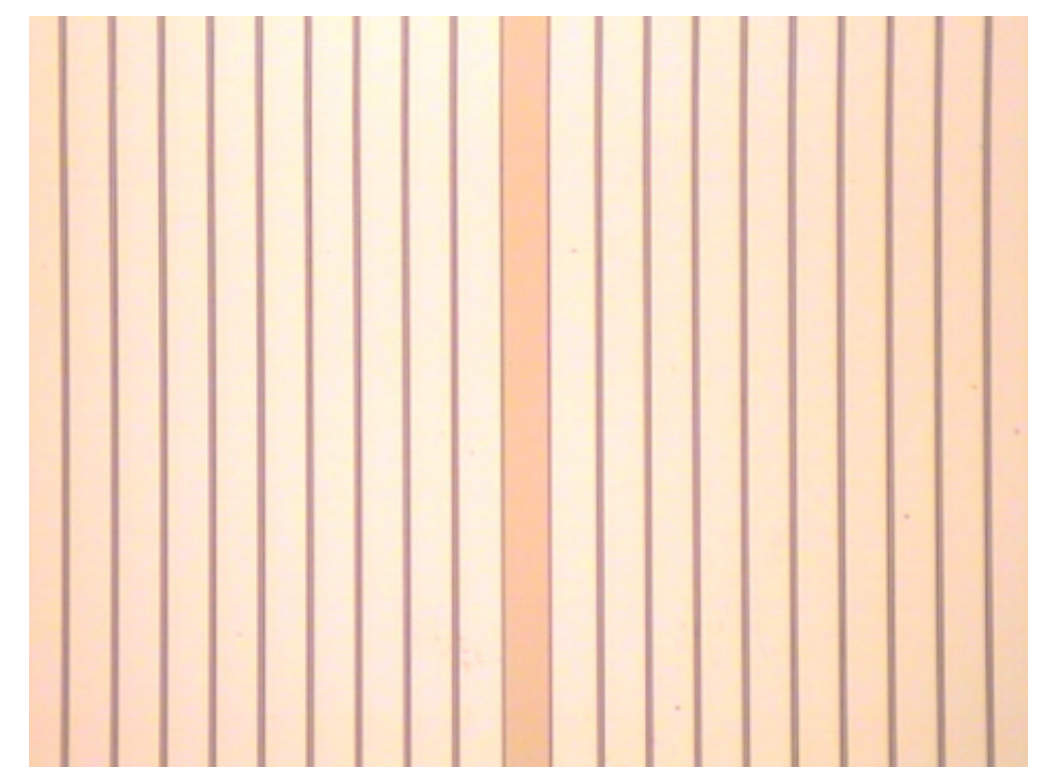

Figure 3.5 $6 \mu \mathrm{m}$ wide waveguide patterns transferred to iron garnet films by UV optical lithography.

\subsubsection{Ion beam etching}

Waveguide ridges are obtained by plasma etching of the transferred waveguide patterns. The instrument used for the etching is a Rib Etch 160 ECR LL ion beam etching system (Tepla,Inc.). To obtain ridge waveguide structures, we employ a physical etching method (dry etching) with Ar gas as the processing gas. 
The desired ridge height is obtained through appropriate etching time. Dry etching process parameters are optimized for magnetic garnet thin films and listed in Table 3-2. The etch depth or the ridge height with respect to etching time is shown Fig 3.6. An interferometer image of an etched waveguides is shown in Fig 3.7.

Table 3-2. CAIBE process parameters

\begin{tabular}{|c|c|}
\hline Process parameter & Value \\
\hline Ar Gas flow rate $(S C C M)$ & 15 \\
\hline Chamber pressure before processing (Torr) & $\sim 10^{-7}$ \\
\hline Chamber Pressure at processing (Torr) & $1-4 \times 10^{-4}$ \\
\hline Accelerating Voltage $(V)$ & 72 \\
\hline Accelerating Voltage $(A)$ & 0.72 \\
\hline Beam Voltage $(\mathrm{kV})$ & 73 \\
\hline Beam Voltage $(A)$ & 0.065 \\
\hline Microwave $(\mathrm{kV})$ & 2.45 \\
\hline Microwave $(A)$ & 400 \\
\hline Magnet $(G)$ & 1250 \\
\hline Magnet $(A)$ & 205 \\
\hline Shutter Current $(V)$ & -0.045 \\
\hline Plasma Intensity $(V)$ & 0.018 \\
\hline Current Density $\left(\mu \mathrm{A} / \mathrm{cm}^{2}\right)$ & $180-210$ \\
\hline
\end{tabular}




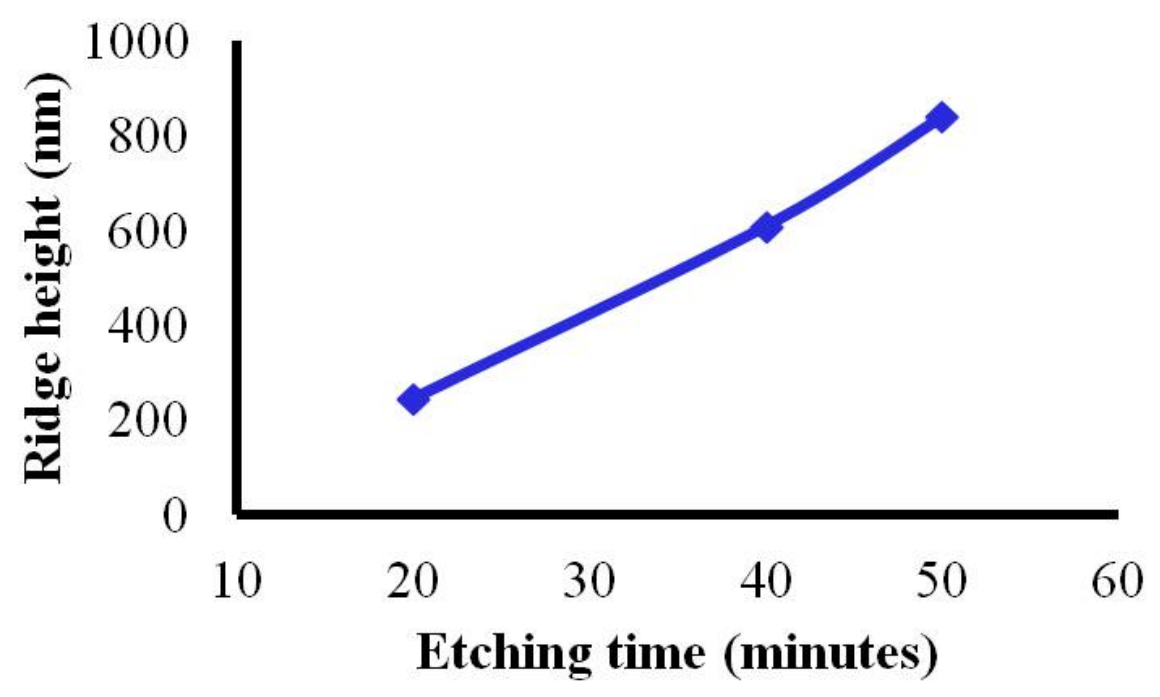

Figure 3.6 Etch rate characterization for Ar ion plasma etching for magnetic garnet thin films with composition $\mathrm{Bi}_{0.8} \mathrm{Gd}_{0.2} \mathrm{Lu}_{2.0} \mathrm{Fe}_{5} \mathrm{O}_{12}$

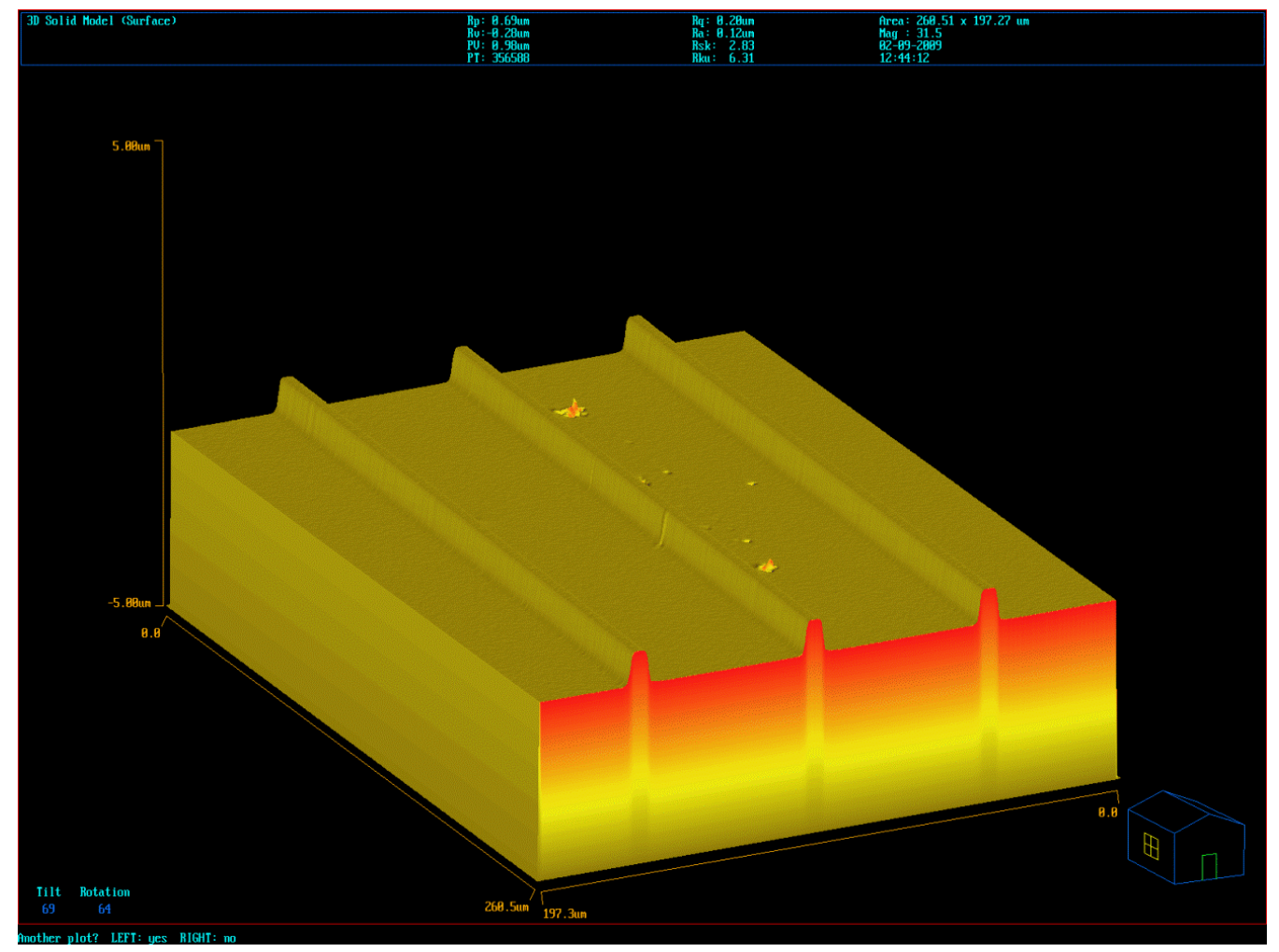

Figure 3.7 An interferrometric image of the waveguides after dry etching. 48 


\subsubsection{Waveguide facet polishing}

After waveguide fabrication, the samples are diced into small pieces $(\sim 2 \mathrm{~mm} \times 10 \mathrm{~mm})$ using a wire saw. Sample dicing with a wire saw makes the waveguide facets too rough for optical testing. To avoid scattering losses and to obtain efficient wave guiding, waveguide facets are polished with a series of diamond lappings. Polishing is achieved in a multi-prep polishing machine with lappings which have $30 \mu \mathrm{m}, 9 \mu \mathrm{m}, 3 \mu \mathrm{m}, 1 \mu \mathrm{m}$ and $0.1 \mu \mathrm{m}$ sized diamond particles. A polished facet of a ridge waveguide is shown in Fig. 3.8 .

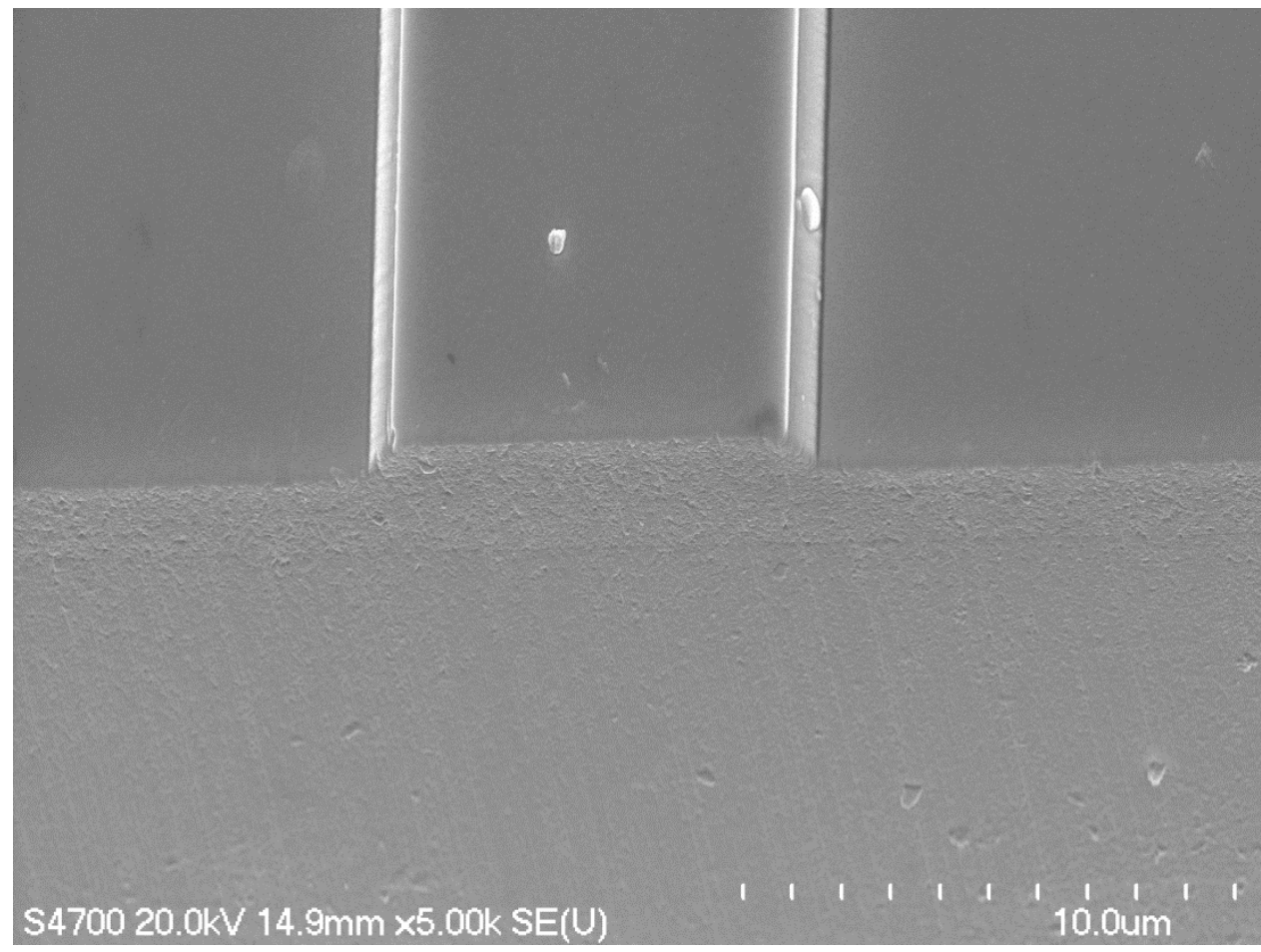

Figure 3.8 Polished facet of a ridge waveguide 


\subsection{Fabrication of photonic crystals}

One-dimensional photonic band gap structures (Bragg gratings) are fabricated onto the ridge waveguides using Hitachi FB-2000A FIB system equipped with NPGS (Nanometer Pattern Generation System). 30keV gallium ions mill down the samples according to the fabrication parameters specified in FIB/NPGS. 200um long gratings with groove depth of $600-700 \mathrm{~nm}$ and grating period ranging from $336 \mathrm{~nm}$ to $355 \mathrm{~nm}$ were used in this study.

\subsubsection{Focused ion beam (FIB) milling}

Focused ion beam fabrication is a mask-less, high-precision nano fabrication technique that has been widely used to produce nanoscale devices in many fields. The FIB has a similar operation principal to a scanning electron microscope. However, the FIB consists of an ion beam instead of an electron beam. Gallium $(\mathrm{Ga})$ is the most commonly-used ion species in the FIB system due to its low meting point $\left(30^{\circ} \mathrm{C}\right)$, low volatility, low vapor pressure and low reactivity with other material [86-88]. In commercial and most research FIB systems, the ion source used is a liquid metal ion source (LMIS) since they provide high brightness and high focused beams. The main components of an FIB system consist of an ion source, condenser lens, objective lens, a beam defining aperture, defection plates and stigmation control poles. The condenser lens and the objective lens define and focus the beam on the sample. The beam diameter and the current are determined by the aperture settings. The deflection plates controls the beam rastering over the sample. A schematic representation of the FIB system is shown in Fig 3.9. FIB is operated at an accelerating voltage between $5 \mathrm{kV}-50 \mathrm{kV}$. The beam diameters and current density of the beam can be controlled through different aperture sizes varying from $6 \mu \mathrm{m}$ to $500 \mu \mathrm{m}$. Table 3-3 shows the beam parameters of the Michigan Tech FIB system. 
Table 3-3. FIB beam parameters

\begin{tabular}{|c|c|c|c|c|}
\hline \multirow{2}{*}{$\begin{array}{c}\text { Aperture size } \\
(\mu \mathrm{m})\end{array}$} & \multicolumn{3}{|c|}{ M0 } & \multicolumn{2}{c|}{ M1 } \\
\cline { 2 - 5 } & $\begin{array}{c}\text { Beam Current } \\
(\mathrm{nA})\end{array}$ & $\begin{array}{c}\text { Beam Diameter } \\
(\mathrm{nm})\end{array}$ & $\begin{array}{c}\text { Beam Current } \\
(\mathrm{nA})\end{array}$ & $\begin{array}{c}\text { Beam } \\
\text { Diameter } \\
(\mathrm{nm})\end{array}$ \\
\hline 500 & - & - & $11-15$ & 1000 \\
\hline 300 & - & - & $4-8$ & 250 \\
\hline 200 & $0.4-0.8$ & 800 & $2-3.5$ & 120 \\
\hline 100 & $0.1-0.3$ & 250 & $0.4-0.8$ & 60 \\
\hline 50 & $0.02-0.05$ & 60 & $0.1-0.3$ & 40 \\
\hline 20 & $0.004-0.01$ & 20 & $0.015-0.04$ & 35 \\
\hline 6 & $0-0.002$ & 10 & $0.001-0.005$ & 35 \\
\hline
\end{tabular}

Since magnetic garnet materials are non-conductive, it is important to coat the samples with a conductive material to facilitate ion dissipation. This is achieved by sputter depositing a $\sim 20 \mathrm{~nm}$ Chromium ( $\mathrm{Cr}$ ) and a $\sim 50 \mathrm{~nm}$ Gold ( $\mathrm{Au}$ ) thin films on magnetic garnet samples prior to FIB fabrication. Depositing the $\mathrm{Cr}$ layer prior to the $\mathrm{Au}$ layer enhances the adhesion of the $\mathrm{Au}$ on the sample. Inadequate charge dissipation causes beam deflection, resulting in curved or misaligned patterns as shown in Fig 3.10. 


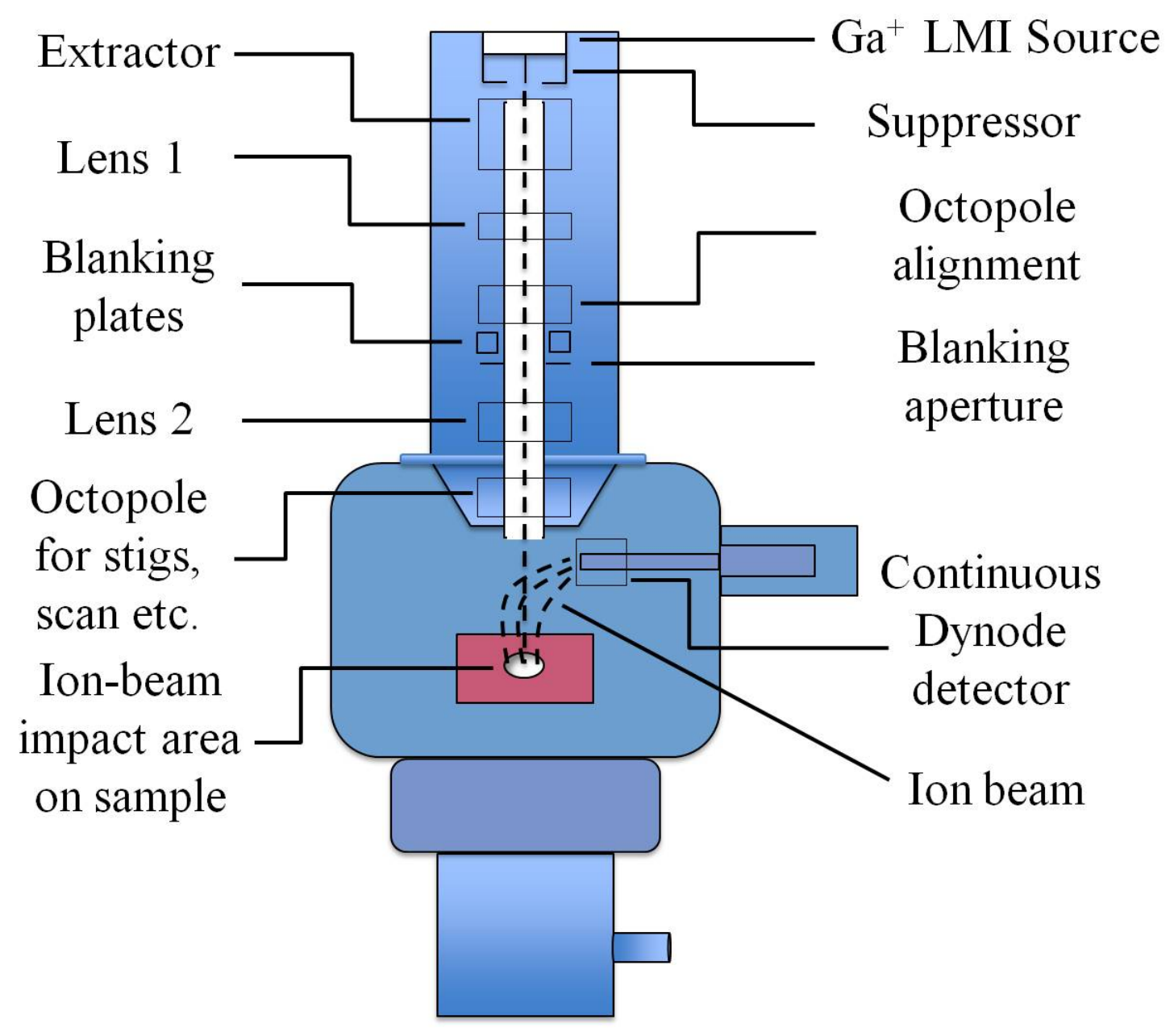

Figure 3.9 Schematic of focused ion beam system 


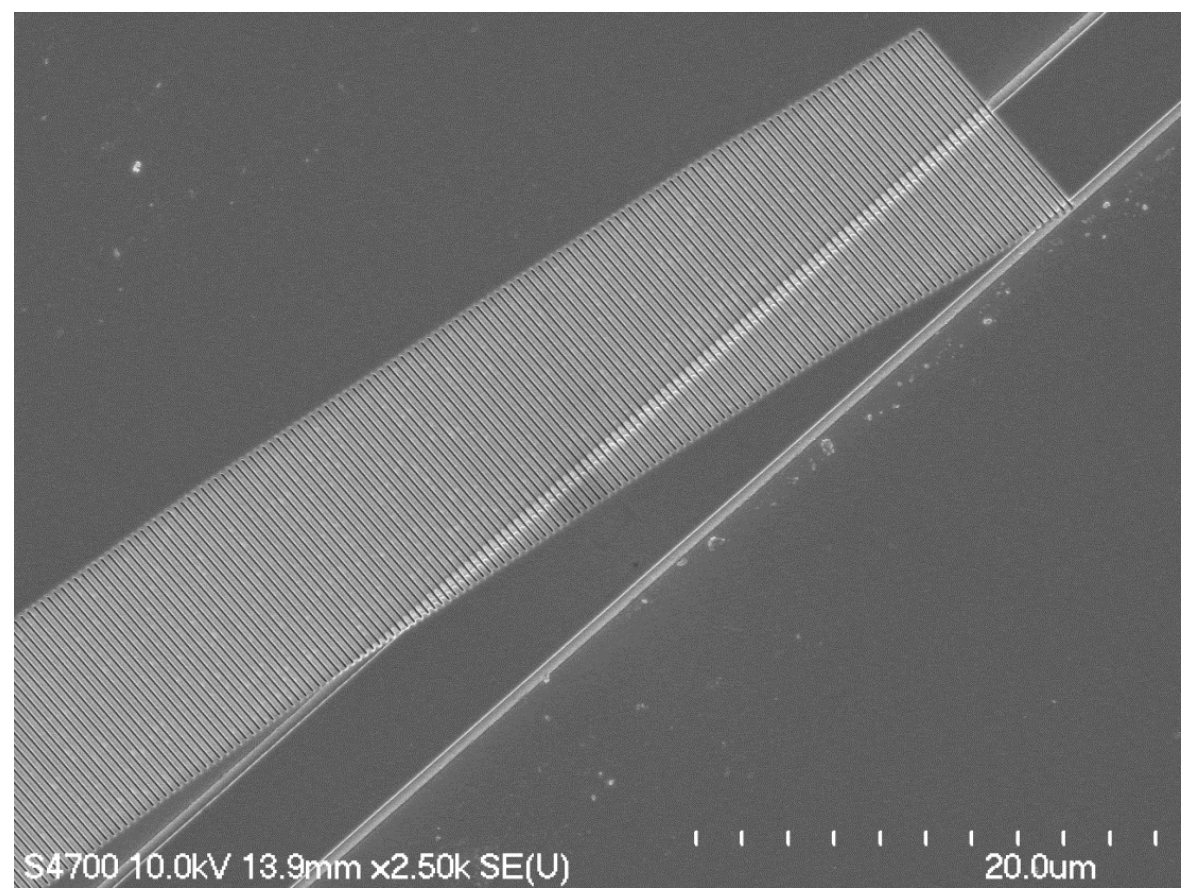

Figure 3.10 SEM micrograph of a misaligned FIB pattern due to charging effects

NPGS software uses a DesignCAD, which provides a user-friendly and flexible environment to create complex nanostructures for direct pattern fabrication using the FIB. In NPGS, a DesignCAD file defines the pattern file and the Run file defines the fabrication parameters. The Run file parameter "Line Dose" (defined below) determines the dwell time for the given center to center distance and the beam current [89].

$$
\text { Line Dose }=\frac{(\text { Beam Current }) \times(\text { Exposure time })}{\text { Center }- \text { to }- \text { Center }}
$$

Here, center to center distance is the distance between adjacent exposure points. In photonic crystal fabrication, the line dose determines the grating groove depth. A SEM image of 1-D PC structure fabricated by FIB is shown in Fig 3.11. 


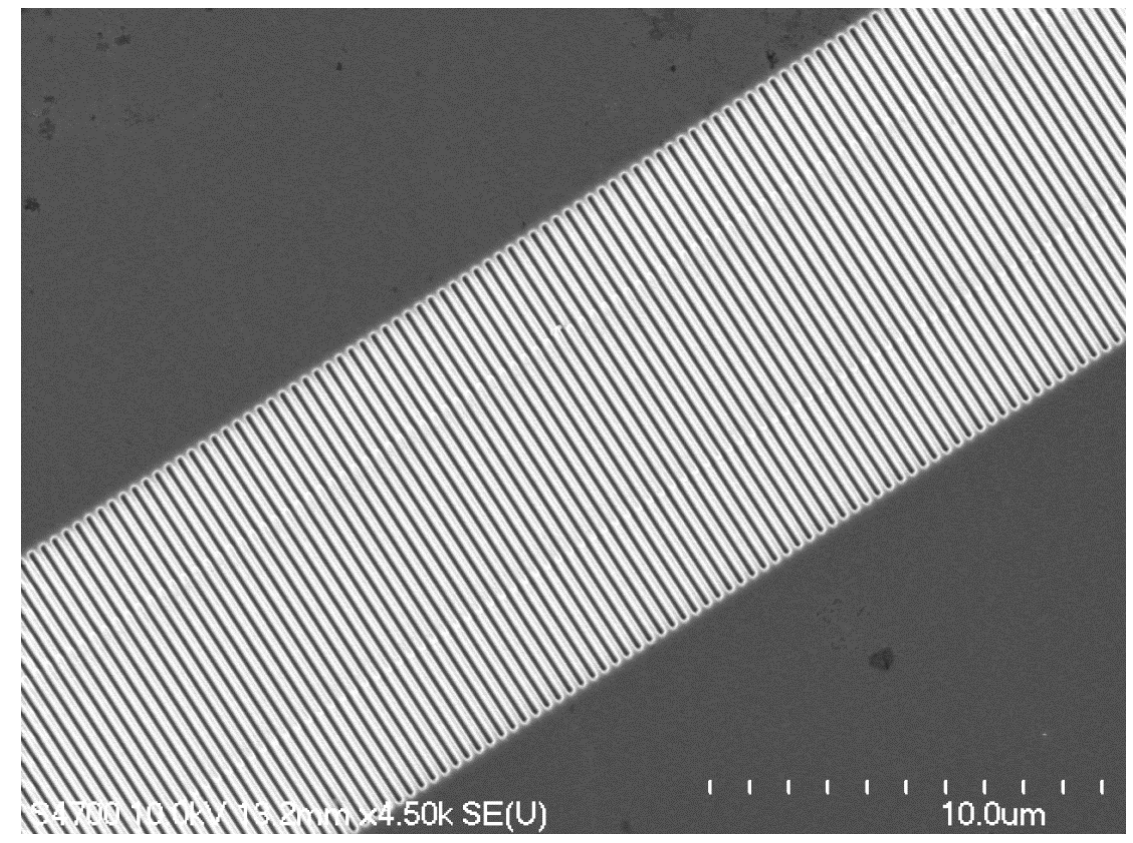

Figure 3.11 SEM micrograph of a one-dimensional photonic crystal fabricated on $\mathrm{Bi}_{0.8} \mathrm{Gd}_{0.2} \mathrm{Lu}_{2.0} \mathrm{Fe}_{5} \mathrm{O}_{12}$ film

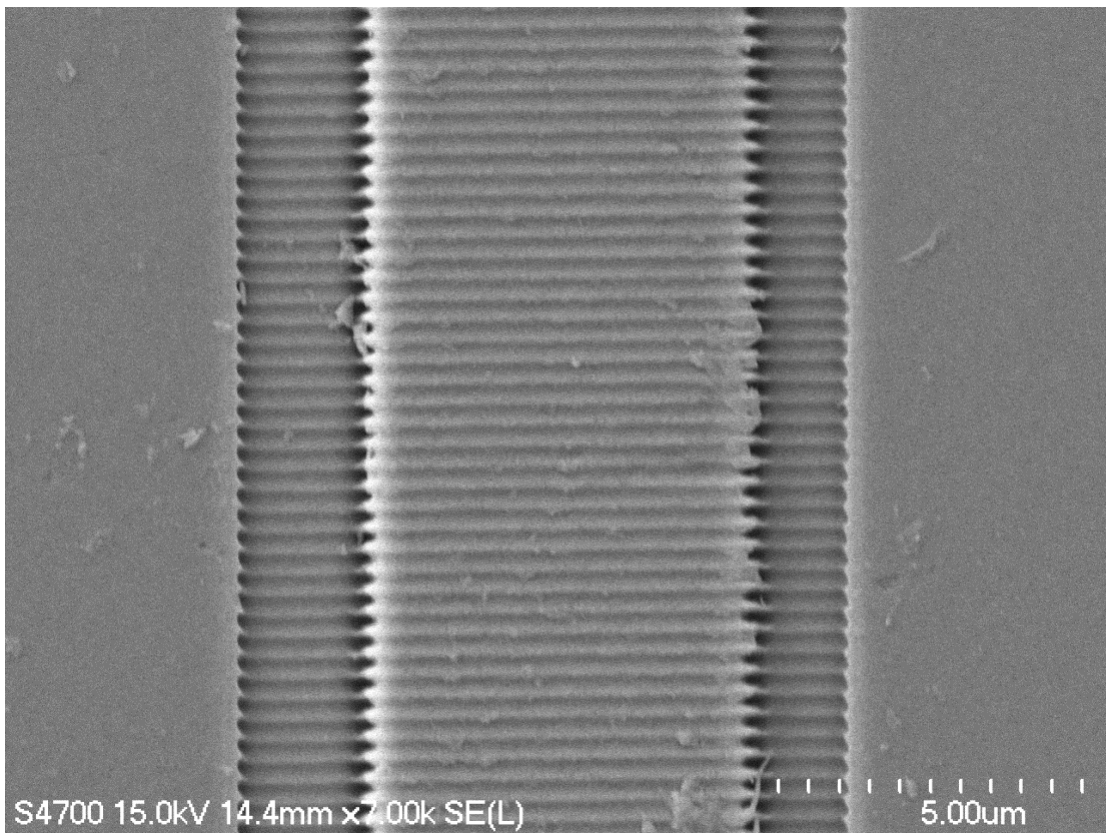

Figure 3.12 SEM micrograph of a one dimensional grating fabricated with underoptimized beam conditions. 
Grating fabrication is very sensitive to beam conditions, such as the focus of the beam on the waveguide, correction for astigmatism and the aperture alignment. Underoptimized beam conditions lead to shallow grating grooves as shown in Fig 3.12.

\subsubsection{Grating depth characterization}

Grating groove depth is controlled by the line dose parameter in NPGS. Therefore, a characterization of grating groove depth versus line dose is required in order to fabricate gratings with desired grove depths. Grating groove depth versus line dose characterization (shown in Fig 3.9) was done by measuring the groove depth in SEM micrographs of gratings which were fabricated using a series of different line doses.

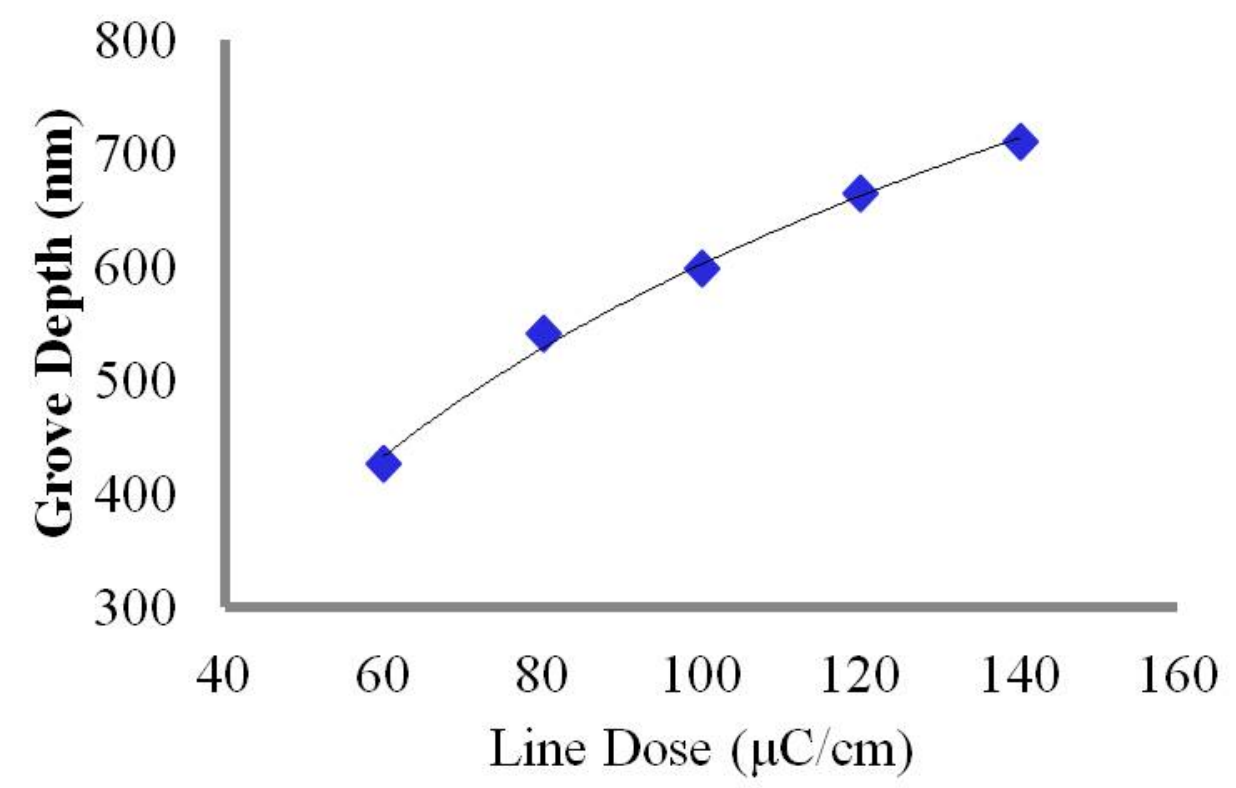

Figure 3.13 FIB milling depth vs line dose for MI-100 beam for magnetic garnet material

In this study, $200 \mu \mathrm{m}$ one-dimensional photonic crystals are fabricated with the specification of 100 line dose to obtain PCs with a $600 \mathrm{~nm}$ groove depth. An SEM micrograph of a cross-section of a grating pattern fabricated with M1-100 beam on Bisubstituted ion garnet is shown in Fig 3.10. 


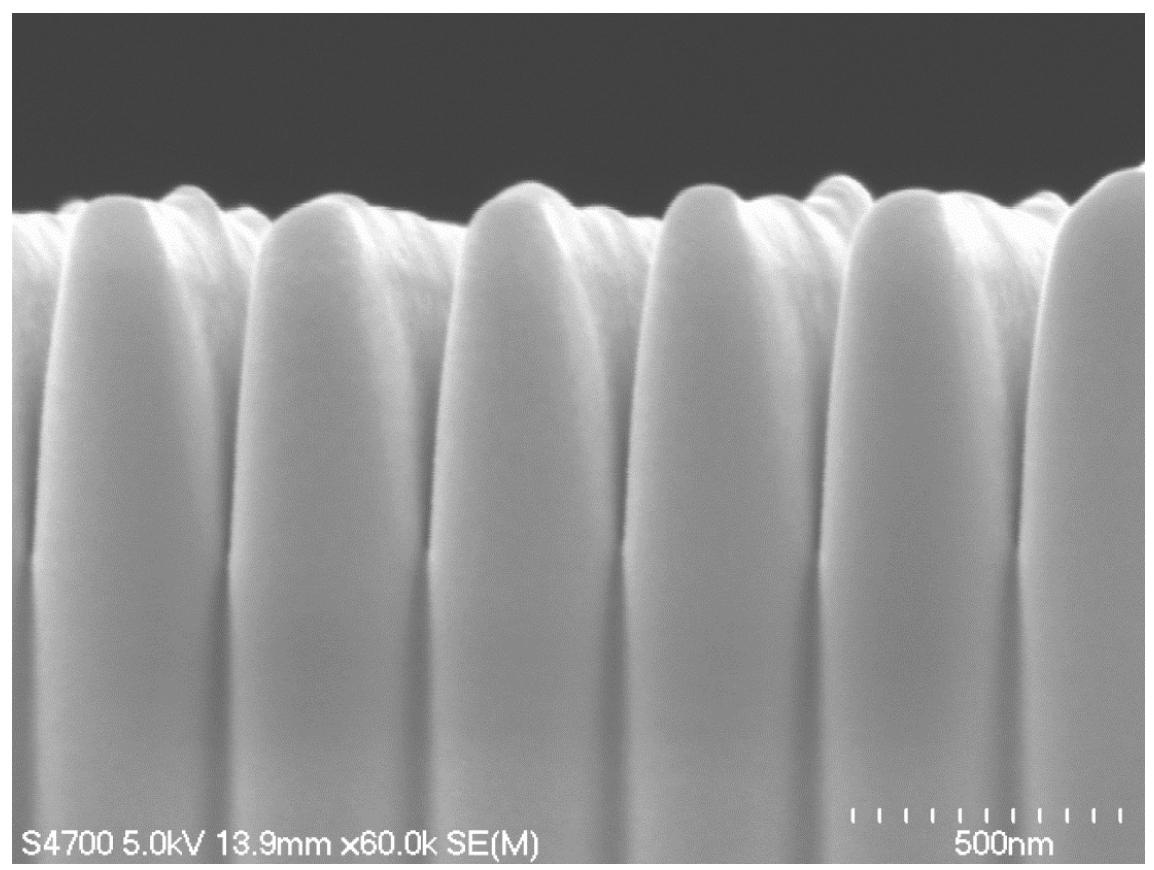

Figure 3.14 SEM micrograph of a grating cross section fabricated on $\mathrm{Bi}_{0.8} \mathrm{Gd}_{0.2} \mathrm{Lu}_{2.0} \mathrm{Fe}_{5} \mathrm{O}_{12}$ film.

\subsubsection{Post Acid Etching}

Photonic crystal structures fabricated with focused ion beam milling require wet etching in ortho-phosphoric acid prior to optical testing. Acid etching reduces the side wall roughness of the grating grooves and at the same time, removes any re-deposition that could occur during FIB milling. The etching process is done by immersing the patterned samples for about 10-15 seconds in a solution of ortho-phosphoric acid which is maintained at $75^{\circ} \mathrm{C}$. The etched samples are promptly washed with de-ionized water to avoid over-etching of the gratings.

Optical transmittance tests performed on a photonic crystal, before and after acid etching, are shown in Fig 3.15. Post-fabrication acid etching vastly improves the transmittance intensity and the coupling efficiency of photonic crystals. 


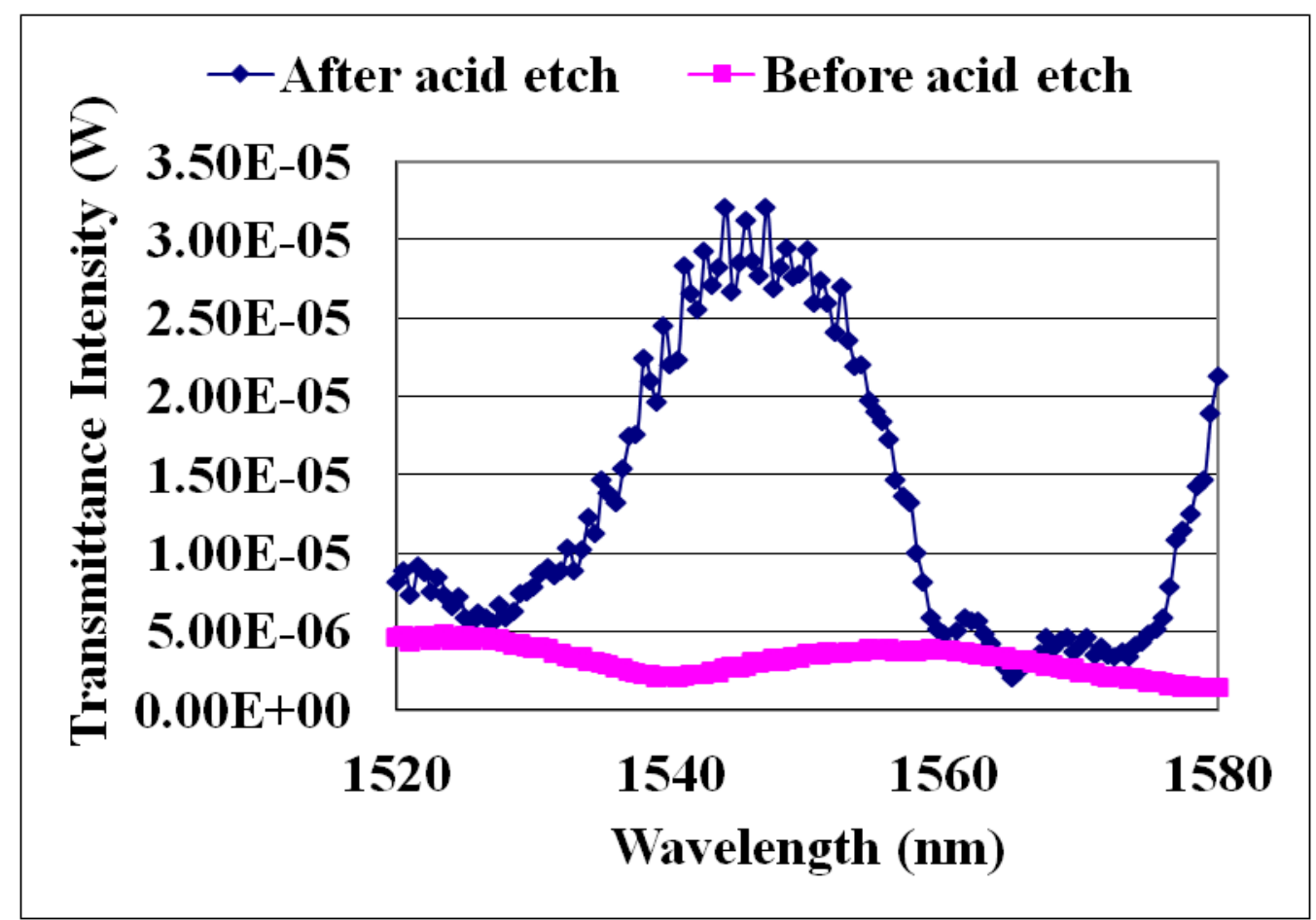

Figure 3.15 Transmittance spectrum of PC structure for pre and post acid etching

\subsection{Optical tests}

Optical transmission and polarization rotation tests are carried out by end-fire fiber coupling from a tunable laser source. Two laser sources have been used in this study: An Ando AQ4321A with wavelength range from 1480nm to $1580 \mathrm{~nm}$ and a Santec laser with wider wavelength range from $1260 \mathrm{nmto} 1630 \mathrm{~nm}$. A fiber from the tunable laser source is connected to a polarization controller, which enables coupling of desired input polarization to the samples. The output beam from the waveguide passes through a $10 \mathrm{x}$ microscope objective and is split into two beams by a $50 \%$ non-polarizing beamsplitter. One beam is sent to the power detector and the other beam is sent to the Hamamatsu infrared camera. Hence, the set-up allows both the beam intensity and the 
beam profile to be obtained simultaneously. The schematic representation of the basic optical set up is shown in Fig 3.16.

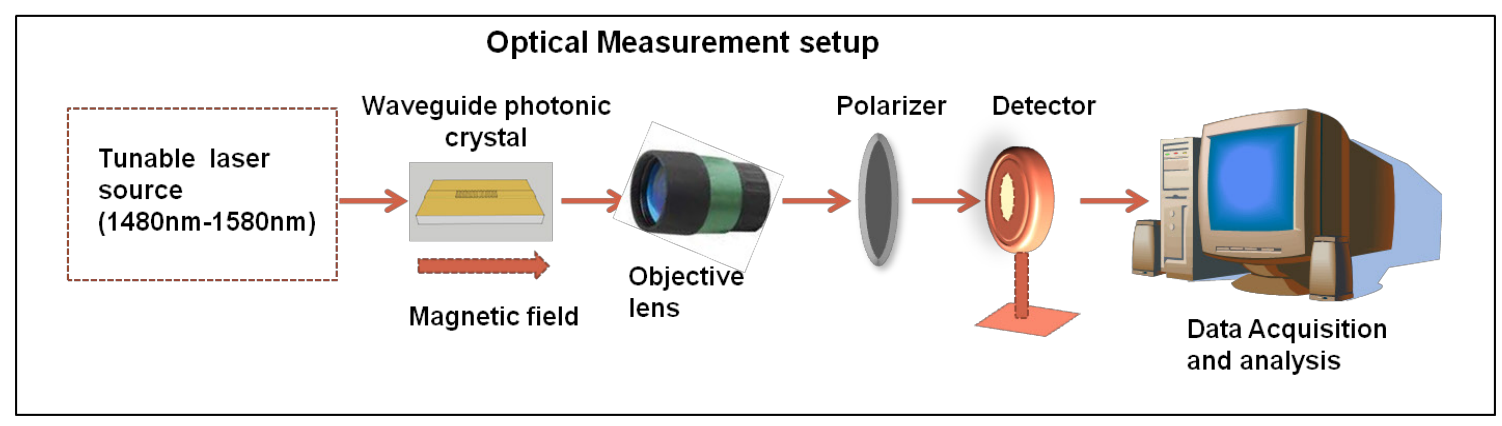

Figure 3.16 Schematic of the optical measurement set-up

\subsubsection{Polarization Rotation}

The polarization rotation measurements are taken in a saturating magnetic field of 3000 e applied collinearly to the waveguide axis. The magnetic field is applied by mounting the sample on a permanent stone magnet. Two techniques, namely a rotating polarizer technique and the Stokes vector technique are employed to extract the polarization state of the PC output.

\subsubsection{Rotating polarizer technique.}

In this technique, the transmitted light intensity through the sample is recorded after having the light pass through a motorized polarizer (analyzer) which is rotated from $0^{\circ}$ to $360^{\circ}$. A graphical representation of the intensity versus the angle of the polarizer axis is shown in Fig 3.17. The rotation of the semi-major axis of the output ellipse with respect to the TE polarized input gives the polarization rotation of the light. 

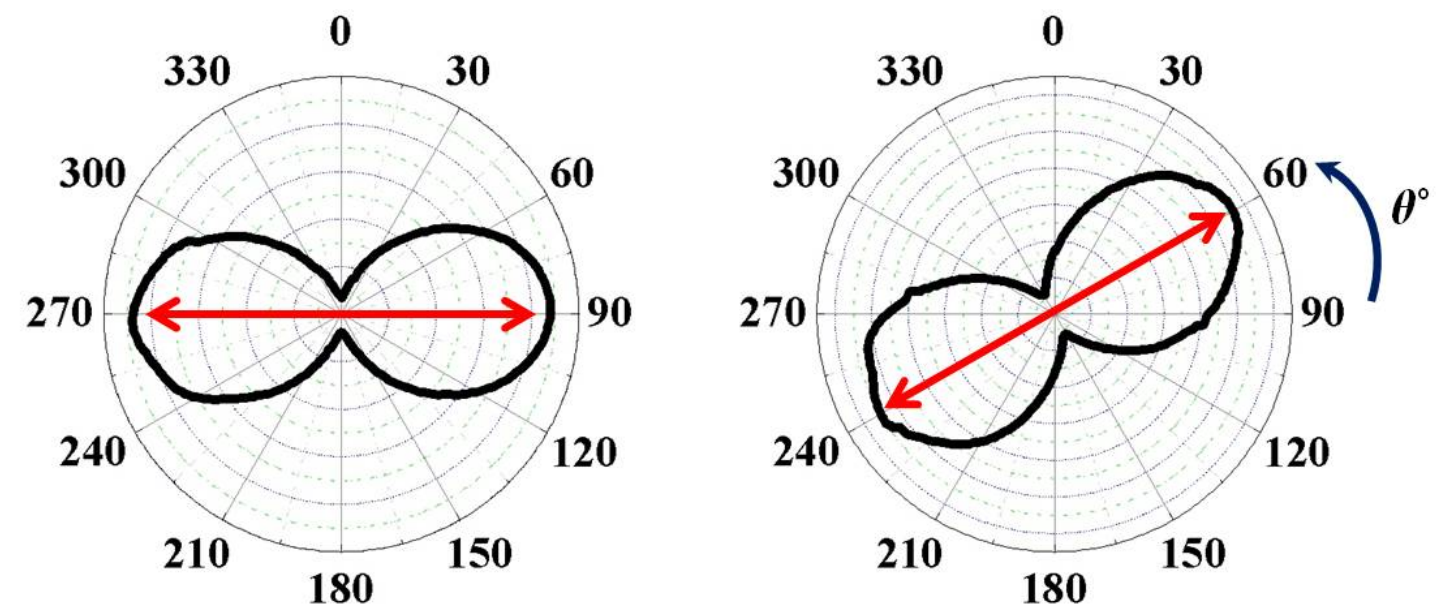

Figure 3.17 In the rotating polarizer technique light intensity vs analyzer rotation angle. Left: for TE input, Right: Output polarization ellipse is rotated by angle $\theta^{\circ}$ with respect to the input $\mathrm{TE}$ light.

\subsubsection{Stokes vector technique}

The complete state of polarization of a light beam can be determined by four Stokes parameters: $\mathrm{S}_{0}, \mathrm{~S}_{1}, \mathrm{~S}_{2}$, and $\mathrm{S}_{3}$, as defined below.

$\mathrm{S}_{0}=$ Total power (polarized and unpolarized) $=I_{0}+I_{90}$

$\mathrm{S}_{1}=$ Power through linear horizontal polarizer $\left(I_{0}\right)$ - power through linear vertical polarizer $\left(I_{90}\right)$

$\mathrm{S}_{2}=$ Power through linear $+45^{\circ}$ polarizer $\left(I_{45}\right)$ - power through linear $135^{\circ}\left(I_{135}\right)$ polarizer

$\mathrm{S}_{3}=$ Power through right circular $(\mathrm{RC})$ polarizer $\left(I_{\mathrm{RCP}}\right)-$ power through left polarizer

The degree of polarization $(D O P)$, ellipticity $(e)$ and orientation of the semi major axis (azimuth $\theta$ of the ellipse) of the polarization ellipse can be obtained as follows [ref]. 


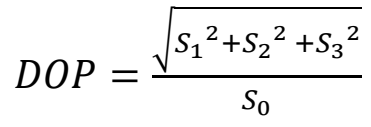

$$
\begin{aligned}
& e=\frac{S_{3}}{S_{0}+{\sqrt{S_{1}{ }^{2}+S_{2}{ }^{2}}}^{2}} \\
& \theta=\frac{1}{2} \arctan \left[\frac{S_{2}}{s_{1}}\right]
\end{aligned}
$$

The linear polarization components at angles $0^{\circ}\left(I_{0}\right), 90^{\circ}\left(I_{90}\right), 45^{\circ}\left(I_{45}\right)$, and $135^{\circ}\left(I_{135}\right)$ are easily measured with a simple polarizer. The right circular polarization and the left circular polarization components of the output light are extracted by a combination of a quarter wave plate and an analyzing polarizer. A schematic of the arrangement of the quarter wave plate and the analyzer for measuring each circular polarization component is shown in Figs. 3.18 and 3.19.

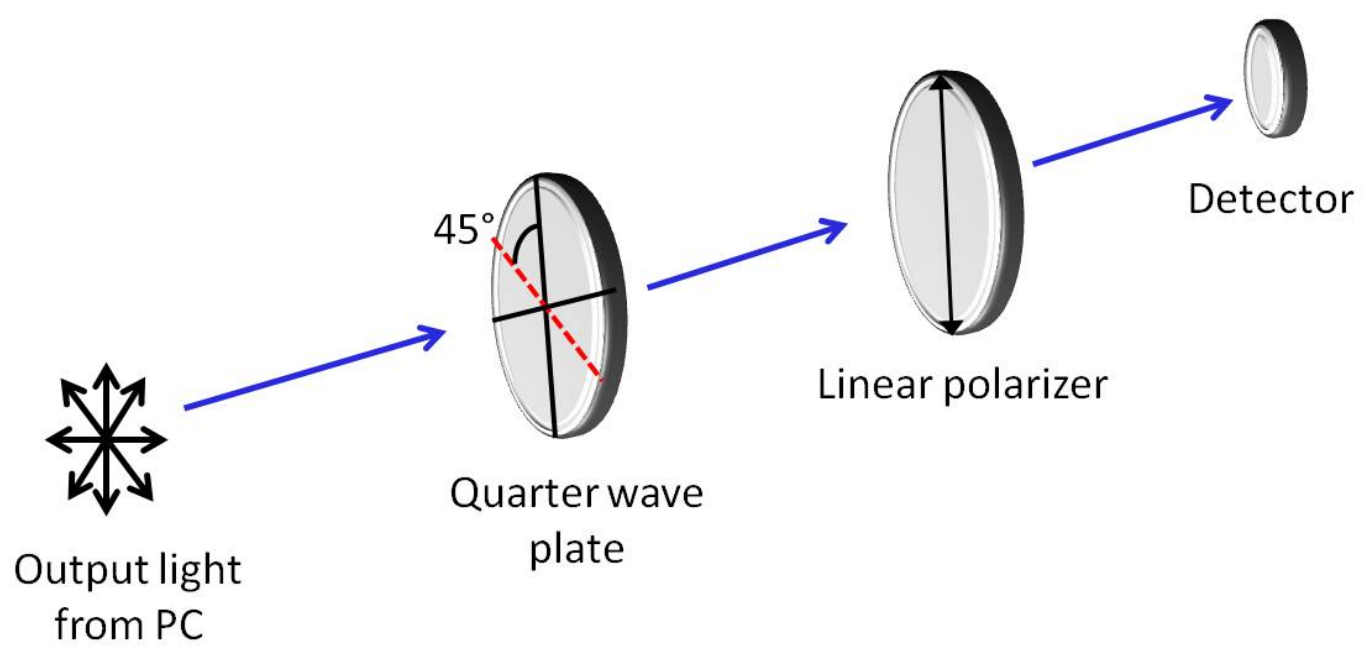

Figure 3.18 Quarter wave and linear polarizer setup to extract $\mathbf{I}_{\mathrm{LCP}}$ 


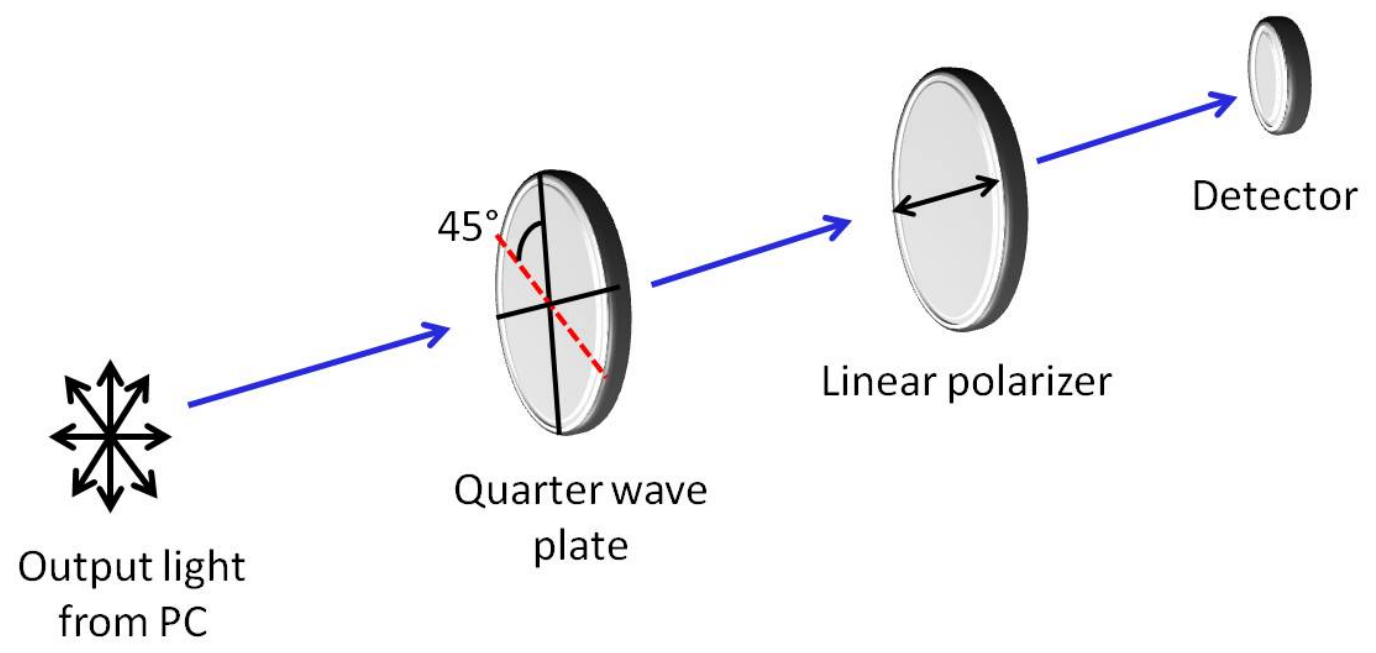

Figure 3.19 Quarter wave and linear polarizer setup to extract $\mathbf{I}_{\mathrm{RCP}}$

The azimuth angle of the polarization ellipse calculated using Eq. 3.3 is transformed into the polarization rotation of the light using the following convention [91].

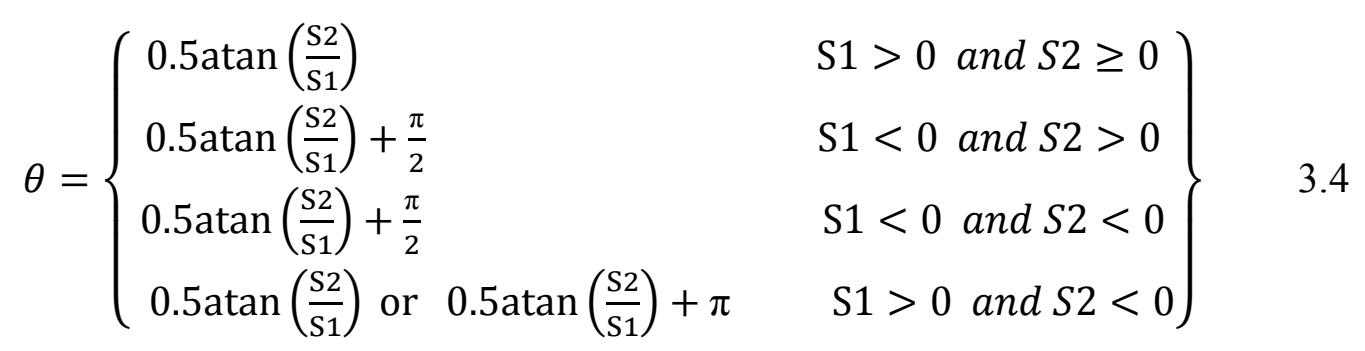

A set of measured Stokes parameters of a Photonic crystal output is given in Appendix A.

\subsection{Synthesis and characterization of Polypyrrole}

Polypyrrole can be synthesized by chemical or electro-chemical oxidative polymerization of the Pyrrole monomer. The electrochemical technique allows for more control of the oxidation process and produces thin Polypyrrole films with higher reproducibility. However, the chemical oxidation technique is easily implemented, less expensive and suitable for mass production [75-79]. In this study, the chemical 
oxidation technique is more suitable mainly due to two reasons. Firstly, the Polypyrrole layer should be selectively deposited only on the photonic crystal which has an area of $10 \mu \mathrm{m} \times 200 \mu \mathrm{m}$.. Secondly, magnetic garnets are dielectric materials which cannot be used as electrodes.

\subsubsection{Chemical oxidation of Polypyrrole}

Chemical synthesis of Polypyrrole was done by chemical oxidation of the Pyrrole monomer. Pyrrole in vapor phase was employed to obtain Polypyrrole thin films on the PC samples. Iron (III) chloride hexahydrate $\left(\mathrm{FeCl}_{3} \cdot 6 \mathrm{H}_{2} \mathrm{O}\right)$ is used as the oxidant. An oxidant solution with $0.1 \mathrm{M}$ concentration was prepared by dissolving $3.4 \mathrm{~g}$ of $\mathrm{FeCl}_{3} \cdot 6 \mathrm{H}_{2} \mathrm{O}$ in acetone. A thin layer of oxidant solution was applied to the photonic crystal by a fiber attached to a micro manipulator shown in Fig 3.19. The oxidant applied PC sample was then exposed to Pyrrole vapor for 10 seconds while the Pyrrole solution was kept on a water bath of $60^{\circ} \mathrm{C}$. A thin black color layer of Polypyrrole was deposited on the PC (Fig 3.20). The thickness of the deposited Polypyrrole layer was controlled by the concentration of the oxidant solution and the Pyrrole exposure time.

Polypyrrole thin films deposited in $\mathrm{Si}$ substrates were used for ellipsometric characterization of Polypyrrole for aq. ammonia exposure. The silicon substrates were cleaned with acetone, IPA and DI water. A solution of $0.6 \mathrm{ml}$ of the Pyrrole monomer dissolved in $100 \mathrm{ml}$ of DI water was then added to the oxidant solution kept on a magnetic stirrer. The mixture turned black indicating the onset of the polymerization process leading to the deposition of the Polypyrrole on Si substrates. Samples with 15 minute deposition time were used for ellipsometric characterization. 


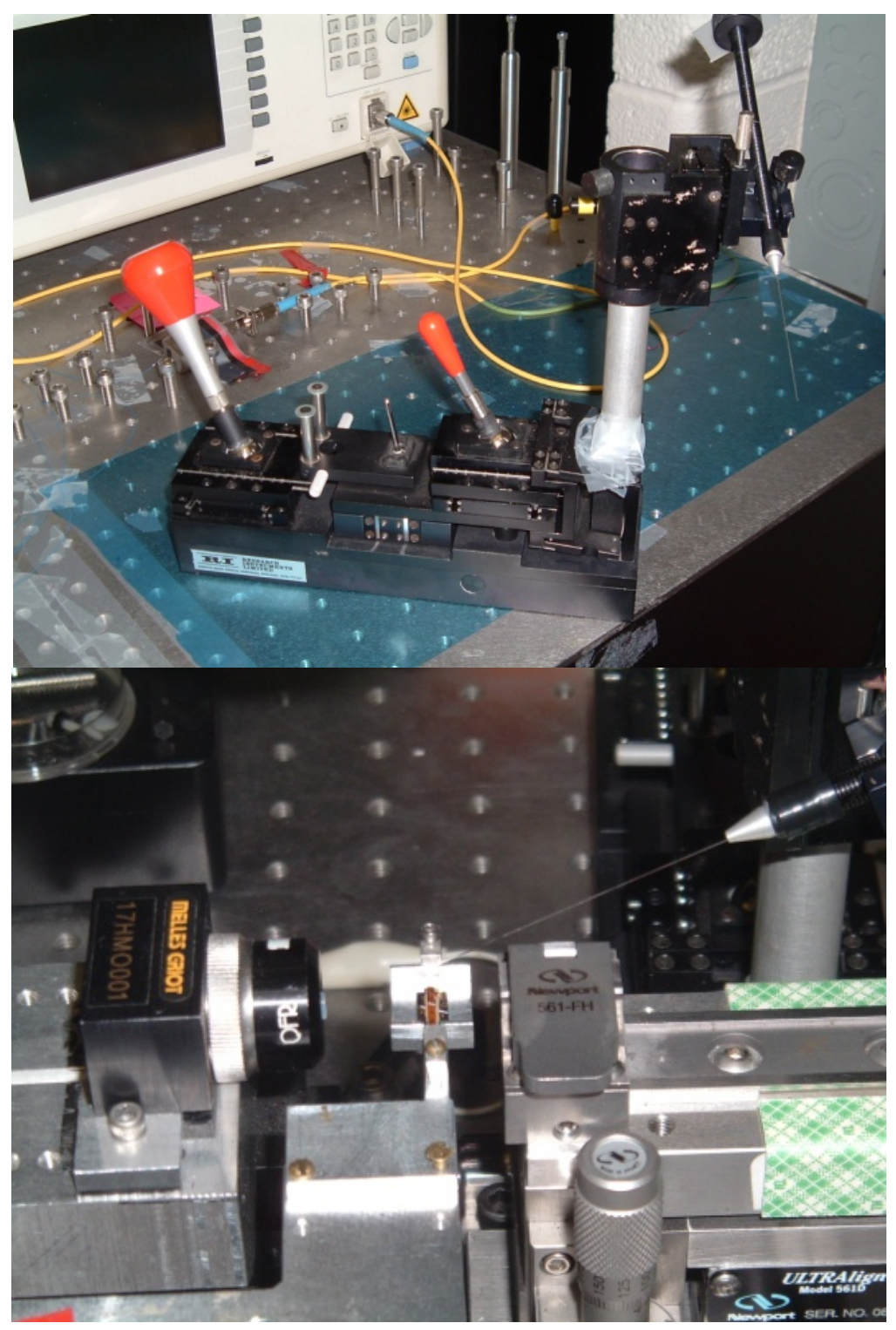

Figure 3.20 The oxidant solution was applied on the $\mathrm{PC}$ with a fiber attached to a micromanipulator. This is performed under an optical microscope in order to apply the oxidant solution only on the $200 \mu \mathrm{m}$ long PC region. 


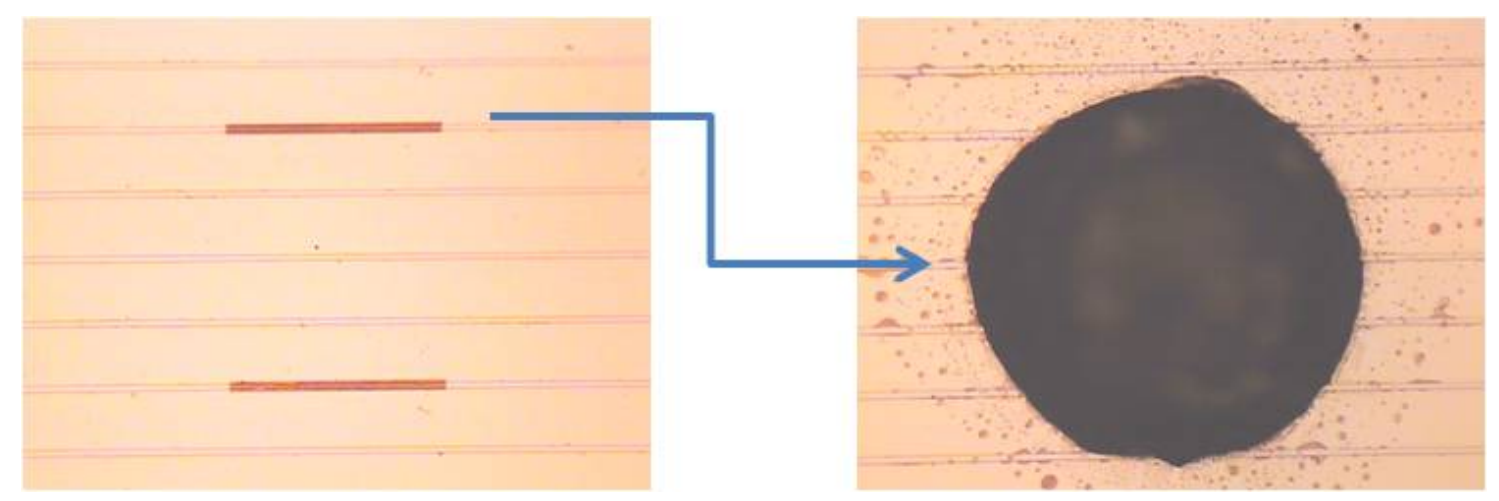

Figure 3.21 Before and after deposition of PPy layer on the photonic crystal. The thickness of the PPy layer is controlled by the oxidant solution concentration and the Pyrrole vapor exposure time

\subsubsection{Ellipsometric characterization of Polypyrrole}

Polypyrrole films deposited on Si substrates were characterized with a Variable Angle Spectroscopic Ellipsometer (VASE). VASE can be used to measure material optical constants, film thickness and surface roughness. The working principle of the VASE is based on Fresnel reflection coefficients. The relation between ellipsometric parameters Psi $(\Psi)$ and Delta $(\Delta)$ and Fresnel reflection coefficients $R_{s}$ and $R_{p}$ is given in Eq. 3.5. $R_{s}$ and $R_{p}$ are reflection coefficients corresponding to S-polarized and P-polarized light respectively.

$$
\tan (\Psi) e^{i \Delta}=\frac{R_{p}}{R_{S}}=\rho
$$

In Variable Angle Spectroscopic Ellipsometry, the ratio of the Fresnel reflection coefficients is recoded with respect to wavelength and incident angle. [92-94] Fig 3.21 shows the typical scheme where incident light is linearly polarized and reflected light is elliptically polarized. 
1. linearly polarized light ...

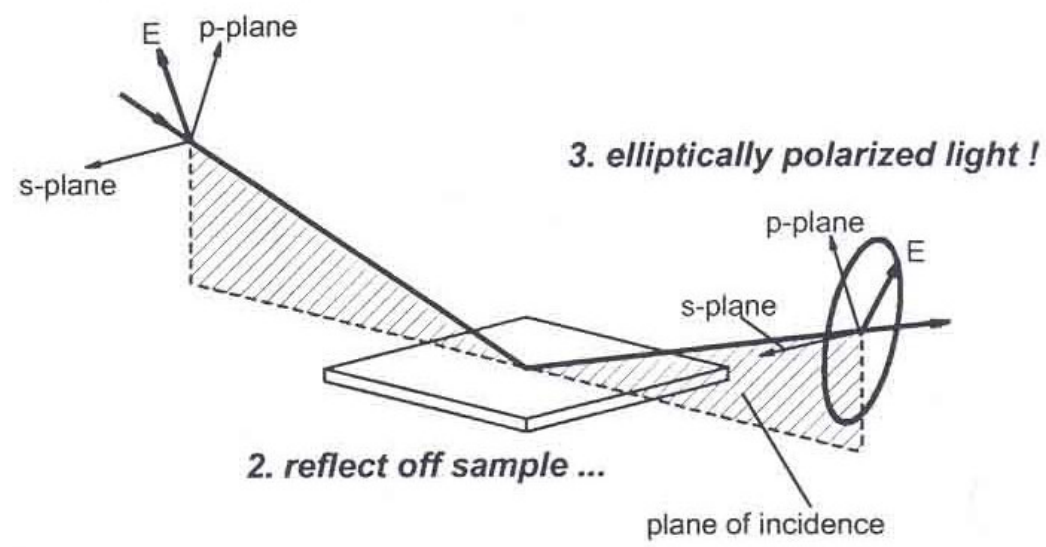

Figure 3.22 Schematic of polarized light and sample interaction in ellipsometer setup

Ellipsometric data for wavelengths in the $300 \mathrm{~nm}$ to $1600 \mathrm{~nm}$ range was collected for the Polypyrrole samples deposited in Si substrates. Ellipsometer analysis software uses the Marquardt-Levenberg algorithm to fit experimental data to a theoretical model. The best fit is acquired when the value of Root Mean Square Error (MSE) is the minimum between the model-fit with the experimental data [94]. 


\section{RESULTS AND ANALYSIS}

In this work we have successfully developed an optical sensing technique using magneto-photonic crystals. The current chapter presents the theoretical and experimental results and the analysis involved in the development of the sensor mechanism, sensor fabrication and testing.

\subsection{Ridge waveguides and mode propagation}

The number of modes that propagate in the ridge waveguide depends on the waveguide geometry and how the light is coupled into the waveguide [28]. A film with thickness $2.71 \mu \mathrm{m}$ shows four waveguide modes propagating in the film slab in prism coupler characterization. We have used beam propagation method to optimize the ridge waveguide structure parameters and beam profile calculations [85]. Simulation results show that for a $2.71 \mu \mathrm{m}$ thick film, a waveguide having a width of $8 \mu \mathrm{m}$ and ridge height between $8 \mu \mathrm{m}-0.12 \mu \mathrm{m}$ delivers more than $90 \%$ of the coupled light in the forward transmittance. When linearly polarized light is launched into the ridge waveguide centered on the input facet, more than $95 \%$ of the guided power propagates in the fundamental mode. Figure 4.1 shows the computed beam profiles of the first three waveguide modes of a ridge waveguide used in this study.

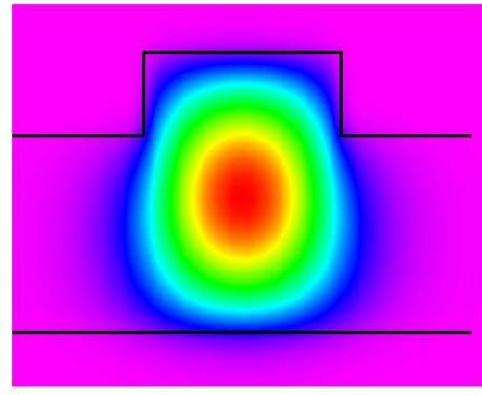

$1^{\text {st }}$ order mode

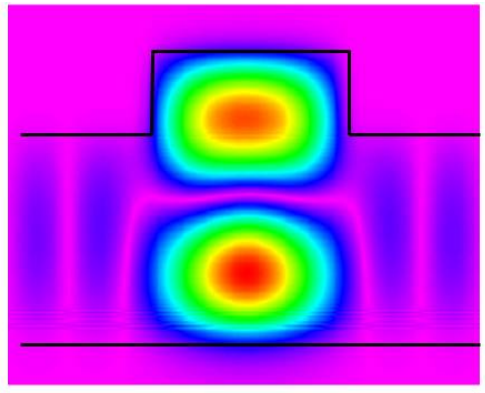

$2^{\text {nd }}$ order mode

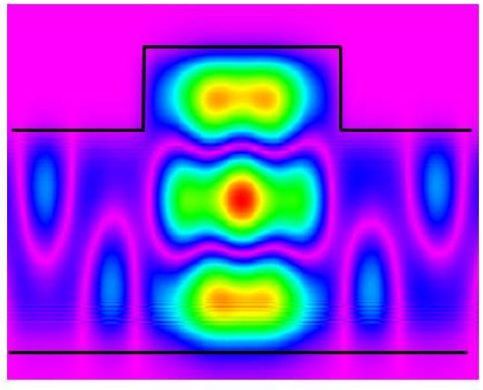

$3^{\text {rd }}$ order mode

Figure 4.1 Computed waveguide mode profiles of a ridge waveguide. 


\subsubsection{Waveguide transmission losses}

The quality of the fabricated waveguides can be determined by evaluating the waveguide characteristics such as propagation constant, propagation losses and light scattering. It is essential to obtain low-loss waveguides in order to achieve high performance in waveguide-based device applications. Some of the main transmission losses in a straight waveguide can be identified as absorption, scattering, mode conversion and reflection loss.

\subsubsection{Absorption loss}

Absorption loss occurs by optical absorption in the waveguide material due to interband transitions, impurity absorption and carrier absorption. Bi-substituted iron garnet films possess excellent optical transparency in the near-infrared wavelength region. However in LPE grown garnet films, optical absorption is significantly increased due to the incorporation of impurities like lead $\left(\mathrm{Pb}^{2+}, \mathrm{Pb}^{4+}\right)$ and platinum $\left(\mathrm{Pt}^{4+}\right)$ ions in to the film during the growth process. $\mathrm{Fe}^{2+}$ and $\mathrm{Fe}^{4+}$ ions generated in the crystal to facilitate the charge balance for these non-three-valent impurities cause a strong increase in the absorption. C. Jovanovic et.al. have shown that the optical absorption in garnets can be lowered by controlling the impurity incorporation into the film and by annealing the films in hydrogen atmosphere [95]. They have shown that the incorporation of impurity ions into the crystal can be controlled by the growth rate and the saturation temperature of the PbO-fluxed melt. In this way a very low optical absorption, below $0.3 \mathrm{~cm}^{-1}$ at $1330 \mathrm{~nm}$ wavelength is achieved by better charge compensation [95]. Similarly, charge compensation may also be established by annealing the films in a hydrogen atmosphere, also leading to a strong reduction of the absorption.

\subsubsection{Scattering loss}

Scattering loss occurs due to the imperfections in the waveguide structure. In this work the surface roughness introduced during the waveguide and PC fabrication process 
could contribute to the scattering loss. This can be reduced by an appropriate postfabrication etching process to remove waveguide and grating grove side wall roughness.

\subsubsection{Coupling loss}

Coupling loss is the power loss that occurs when coupling the light from one optical media to the other. Reflection (Fresnel) losses and mode conversion losses contribute to coupling loss.

When light is incident on an interface of two homogeneous media with different refractive indices, some of the light gets reflected at the interface. At normal incidence the Fresnel reflection loss is given by,

$$
L_{f}=10 \cdot \log \left(\frac{n_{1}-n_{2}}{n_{1}+n_{2}}\right)^{2} d B
$$

where $n_{1}$ and $n_{2}$ are the refractive indices of the two media. For magnetic garnet waveguides with refractive index 2.31, the Fresnel reflection loss is estimated to be $8.05 \mathrm{~dB}$.

Mode mismatch arises mainly due to geometrical cross-section and waveguide-corecladding-indices mismatch between the fiber and the waveguide. The variation in the mode-size, mode shape, and effective-index mismatch between them induces coupling to radiation modes and back reflection. However, reflection and coupling loss can be minimized by applying anti-reflection coating at the facet or realizing tapered waveguides.

\subsection{Magneto-optic Bragg gratings}

Polarization effects enhanced by one-dimensional magneto-photonic crystals (Bragg gratings) play the key role in the detection mechanism of the sensor technique developed here. Therefore, fabricating high quality photonic crystals was a critical part in the sensor development process. Focused Ion Beam technology allows fabricating 
high quality photonic crystals with optimized process parameters. The photonic crystal fabrication process is discussed in detail in section 3.3.

One-dimensional photonic crystals fabricated on ridge waveguides show multiple band gaps in the output transmittance spectrum due to contra-directional coupling of the forward fundamental to reflected higher-order waveguide modes. The transmittance output of a PC is shown in Fig. 4.2. The Bragg scattering condition for mode coupling is given by $\lambda=\left(n_{f}+n_{b}\right) \Lambda$ where $n_{f}$ and $n_{b}$ are the refractive indices of the forward and backward propagating waveguide modes [11].

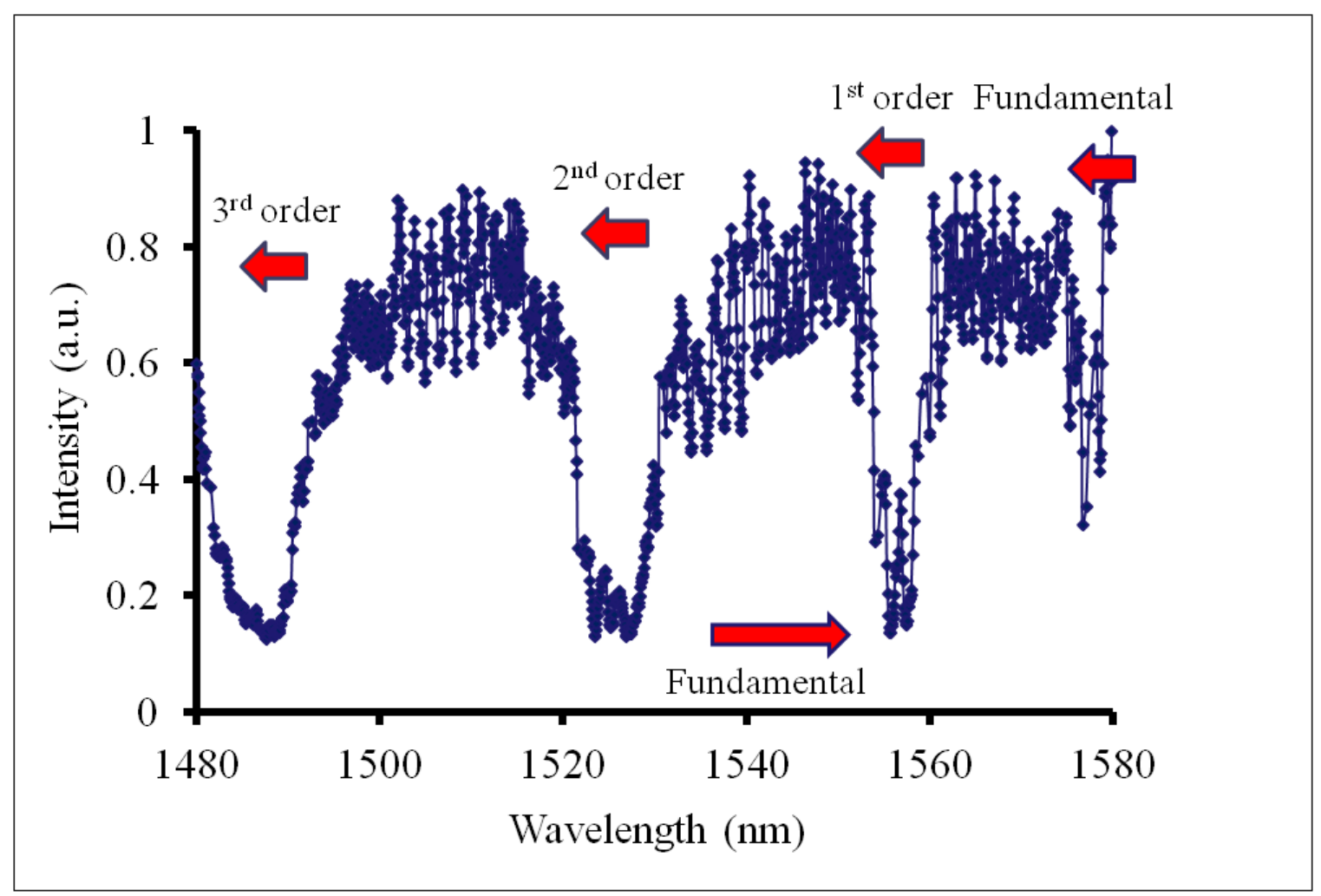

Figure 4.2 The transmittance spectrum of a one-dimensional photonic crystal with period $337 \mathrm{~nm}$ fabricated on a $2.71 \mu \mathrm{m}$-thick $\mathrm{Bi}_{0.8} \mathrm{Gd}_{0.2} \mathrm{Lu}_{2.0} \mathrm{Fe}_{5} \mathrm{O}_{12}$ film. 


\subsubsection{TE and TM band gaps}

A magnetic garnet film of thickness $2.71 \mu \mathrm{m}$ supports four TE and TM modes in the film slab. The band gaps are observed for both TE and TM input polarized light; however, TE gaps are more prominent. The combination of material birefringence in the film and the geometrical birefringence induced by the asymmetric ridge waveguide structure produce a non-zero linear birefringence in mode indices in the system. Therefore TE and TM modes propagate with different propagation constants in the waveguide, leading to different spectral positions for TE and TM bandgaps in the output spectrum. The stop bands are more clearly observable when a transverse (perpendicular to the waveguide axis) magnetic field is applied, where no magneto-optic coupling occurs between TE and TM modes. Figure 4.3 shows the TE and TM mode band output for a grating fabricated on a $2.71 \mu \mathrm{m}$-thick ridge waveguide on $\mathrm{Bi}_{0.8} \mathrm{Gd}_{0.2} \mathrm{Lu}_{2.0} \mathrm{Fe}_{5} \mathrm{O}_{12}$ film.

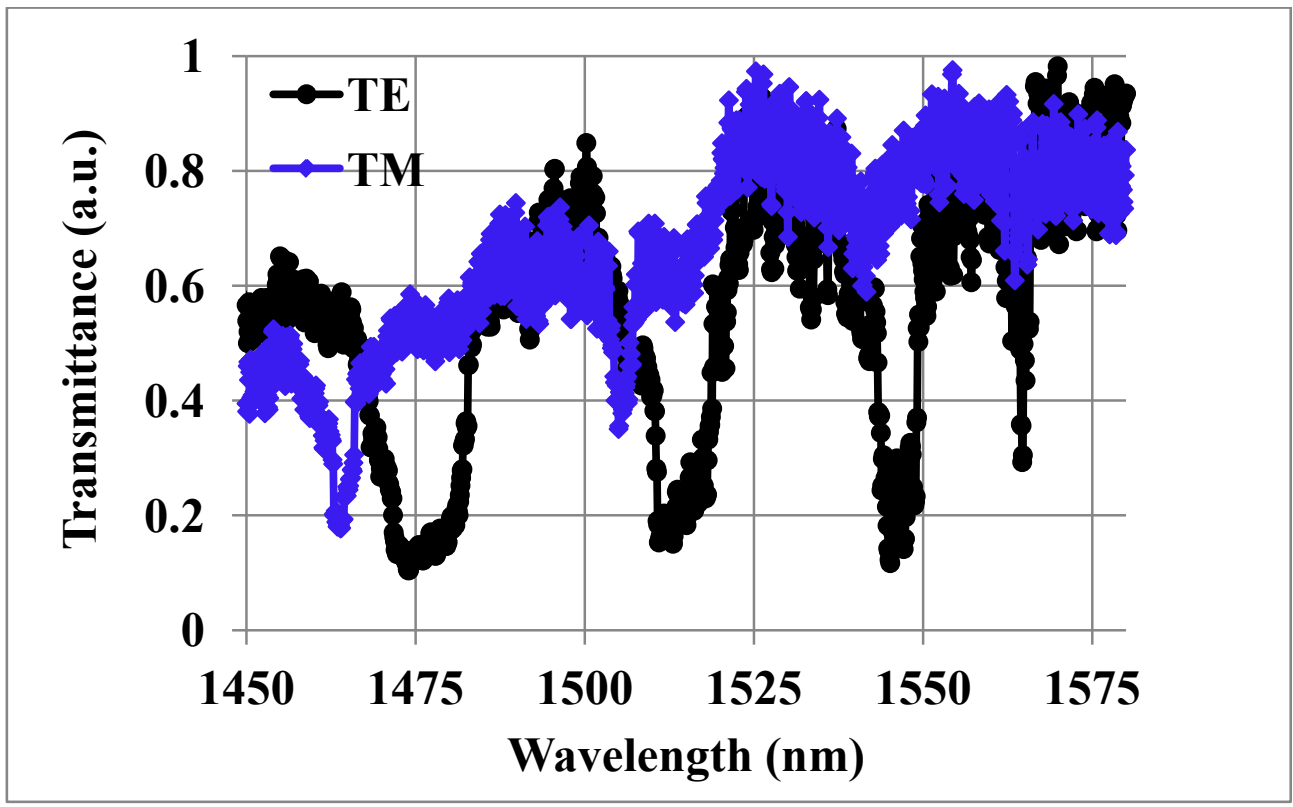

Figure 4.3 Stop bands corresponding to transverse electric and transverse magnetic input polarization states. 
Since the linear birefringence increases for higher-order modes, the separation between TE and TM band gaps increases. The mode indices of the TE and TM modes and the center wavelengths of each band gaps are listed in Table 4-1.

Table 4-1. Prism coupler measured refractive index data and the corresponding

\begin{tabular}{|c|c|c|c|c|c|}
\hline & Film index & $\begin{array}{c}\text { Fundamental } \\
\text { mode }\end{array}$ & $\begin{array}{c}1^{\text {st }} \text { order } \\
\text { mode }\end{array}$ & $\begin{array}{c}2^{\text {nd }} \text { order } \\
\text { mode }\end{array}$ & $\begin{array}{c}3^{\text {rd }} \text { order } \\
\text { mode }\end{array}$ \\
\hline $\begin{array}{c}\text { TE } \\
\text { polarization }\end{array}$ & 2.3182 & 2.30411 & 2.2616 & 2.1896 & 2.08661 \\
\hline $\begin{array}{c}\text { TM } \\
\text { polarization }\end{array}$ & 2.3192 & 2.30362 & 2.25627 & 2.17659 & 2.06505 \\
\hline $\begin{array}{c}\text { Birefringence } \\
\text { TE gap center wavelength } \\
(\mathrm{nm})\end{array}$ & 1564.6 & 1547.1 & 1514.0 & 1476.8 \\
\hline $\begin{array}{c}\text { TM gap center wavelength } \\
\text { (nm) }\end{array}$ & 1563.6 & 1541.0 & 1505.0 & 1463.0 \\
\hline $\begin{array}{c}\text { TE and TM band gap } \\
\text { separation (nm) }\end{array}$ & 1.0 & 6.1 & 9.00533 & 13.8 \\
\hline
\end{tabular}

\subsection{Polarization rotation in magneto-optic Bragg gratings}

Previous work by Professor Levy's research group has shown that there is a significant enhancement in polarization rotation near the band edges in Bragg waveguides in gyrotropic systems for coupling forward traveling fundamental to backscattered higherorder modes [11-15]. Polarization rotation enhancement has also been demonstrated with one-dimensional MPCs composed of magnetic-garnet layered stacks [29,56-58]. In the latter the enhancement mechanism is explained by photon trapping in the resonant 
cavity. In the resonant cavity, light undergoes multiple reflections by the cavity walls that increase the optical path length of light in the magnetic media leading to an increase in net Faraday rotation. However, gyrotropic Bragg waveguides differ from these systems due to the presence of linear birefringence in them. Linear birefringence tends to suppress the Faraday rotation, and yet large magneto-optic polarization rotations, responsive to magnetic field reversals, have been observed even for significant linear birefringence in the backscattered waves. Most importantly, the enhanced rotation is observed in gratings without resonant cavities as similar to the ones with resonant cavities. Therefore, the polarization rotation enhancement in magnetooptic Bragg waveguides involves a different mechanism from photon trapping. Experimental and theoretical investigations on these systems suggest that this enhancement is due to the combined effect of Faraday rotation and birefringence in waveguide magneto-photonic crystals. There is no rotation for pure linear birefringence as shown by transverse magnetization measurements in Fig. 4.4.

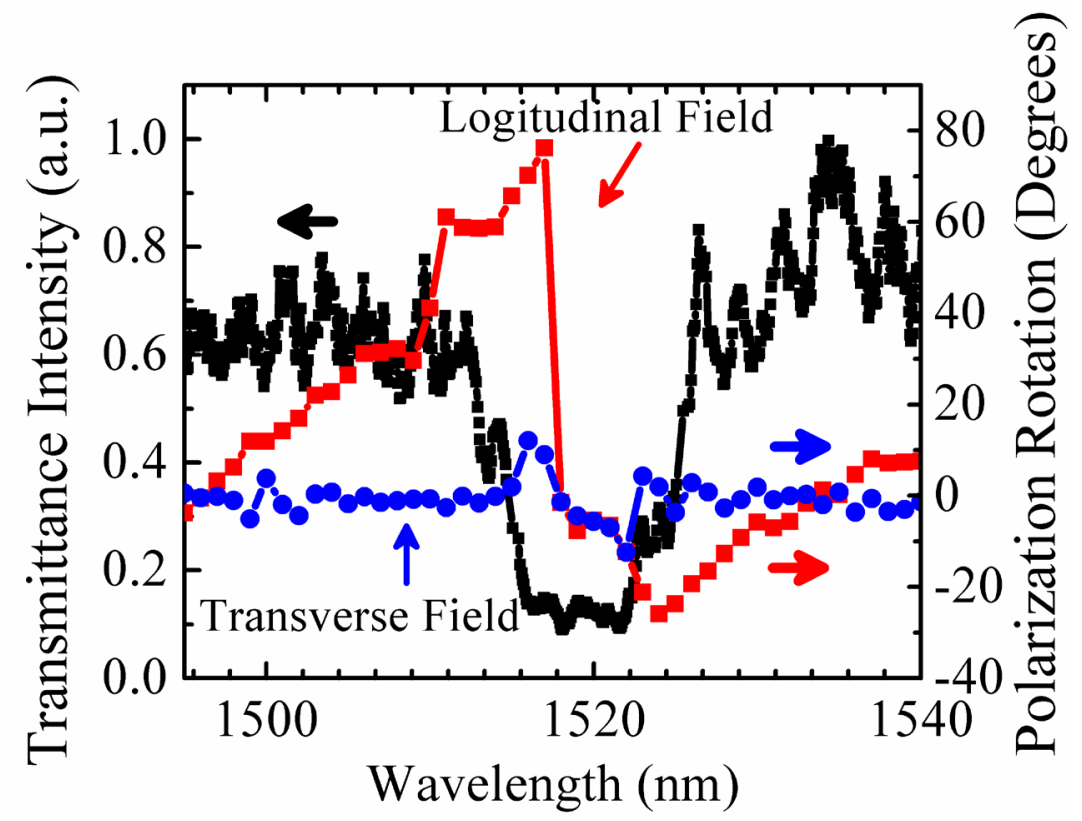

Figure 4.4 Polarization rotation measured for longitudinal magnetization (red) and for transverse magnetization (blue) showing large rotation for longitudinal magnetization and negligible rotation for a transverse field. 
Linear birefringence by itself yields no contribution for TE and TM inputs. Similarly, very little rotation is observed for pure FR by fundamental to backscattered fundamental (at $\sim 1575 \mathrm{~nm}$ ) where linear birefringence (of the fundamental mode) is negligibly small (Fig. 4.5).

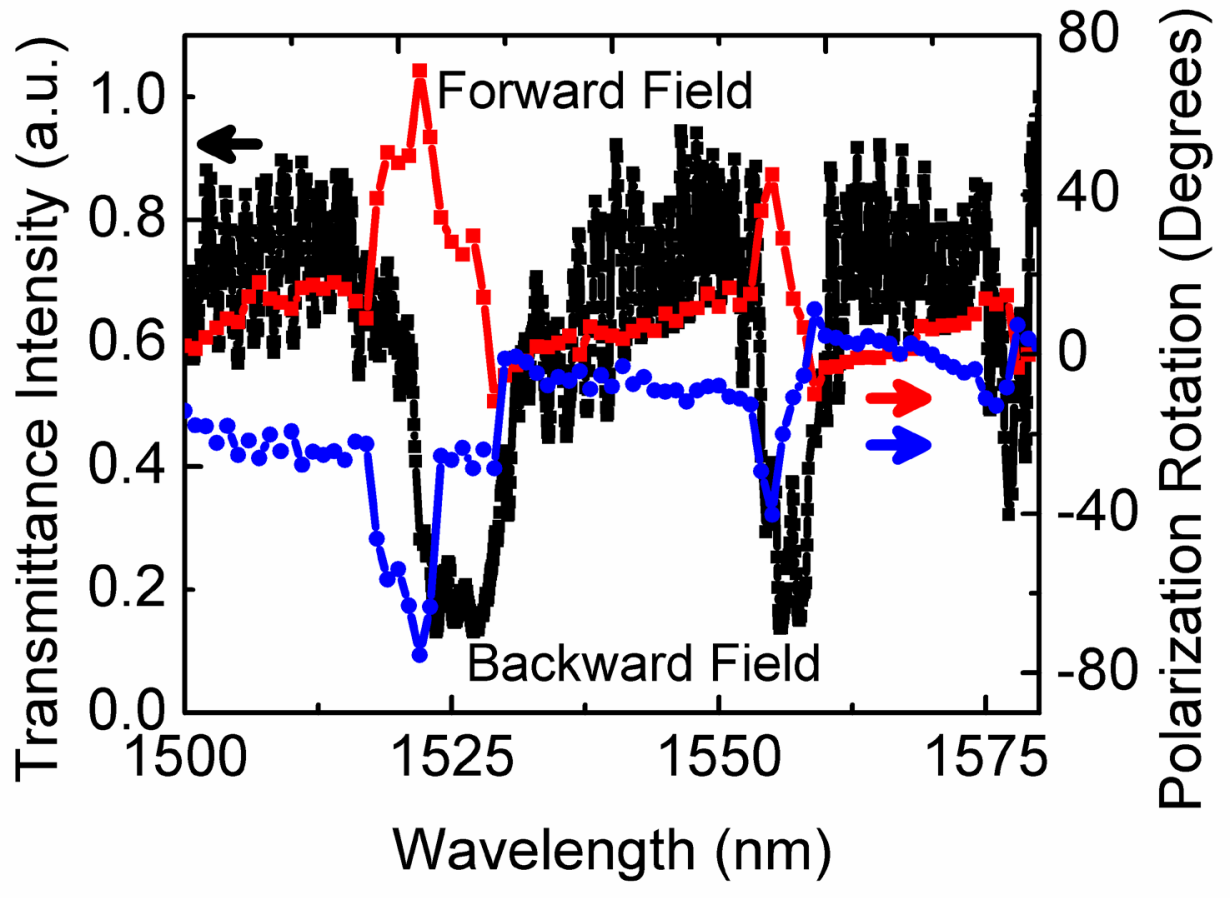

Figure 4.5 Polarization rotation measured for fundamental, first and second backscattered-order band gaps for longitudinal forward magnetization (red) and longitudinal backward magnetization (blue). These data show that the near bandedge polarization rotation increases with back-scattered mode order, and that the rotation is magneto-optic in character.

Linear birefringence occurs naturally in magneto-optic waveguides as a consequence of geometrical confinement and stress. Therefore, the simultaneous presence of optical gyrotropy and linear birefringence in these systems leads to elliptically polarized normal modes in the guide parameterized by $\hat{e}_{ \pm}=\frac{1}{\sqrt{2}}\left(\begin{array}{c}\cos \alpha \pm \sin \alpha \\ \pm i \cos \alpha-i \sin \alpha \\ 0\end{array}\right)$, where 
$\tan (2 \alpha)=\frac{n_{T E}^{2}-n_{T M}^{2}}{2 g}[12] . n_{T E}, n_{T M}$ and $g$ are the transverse-electric mode index, transverse-magnetic mode index and the optical gyrotropy parameter of the medium respectively. The coupling of elliptically birefringent normal modes in magnetophotonic systems leads to formation of gyrotropic band gaps inside the Brillouin zone and strong polarization effects. A detailed theoretical background on formation of gyrotropic band gaps in elliptically birefringent magneto optic media can be found in references $[12,13]$.

\subsubsection{Experiment and theoretical evidence}

A computational analysis and experimental evidence on gyrotropic band gap formation and enhanced polarization rotation in magneto-optic Bragg waveguides are presented in this section. The computational analysis is based on the theoretical model presented in section 2.3.5. The model consisted of a birefringent magneto-optic layered-stack structure with a bilayer unit cell. The gyrotropic band gaps are formed primarily due to contra directional coupling of dissimilar waveguide modes in the system. The model is designed to capture this essential feature of magneto-optic Bragg waveguides by introducing a linear combination of elliptical normal modes in the adjacent layers of the model. Forward- and backward-traveling local modes are allowed to differ in refractive index and elliptical-polarization state to account for the contra-directional coupling between modes of different orders in the waveguide.

The model calculates the band structure for the elliptically birefringent magnetooptic layered system and the corresponding polarization rotation (Bloch mode orientation). The study compares the experimental and computed band structure and polarization rotations for multiple Bragg waveguides with and without resonant cavities. 


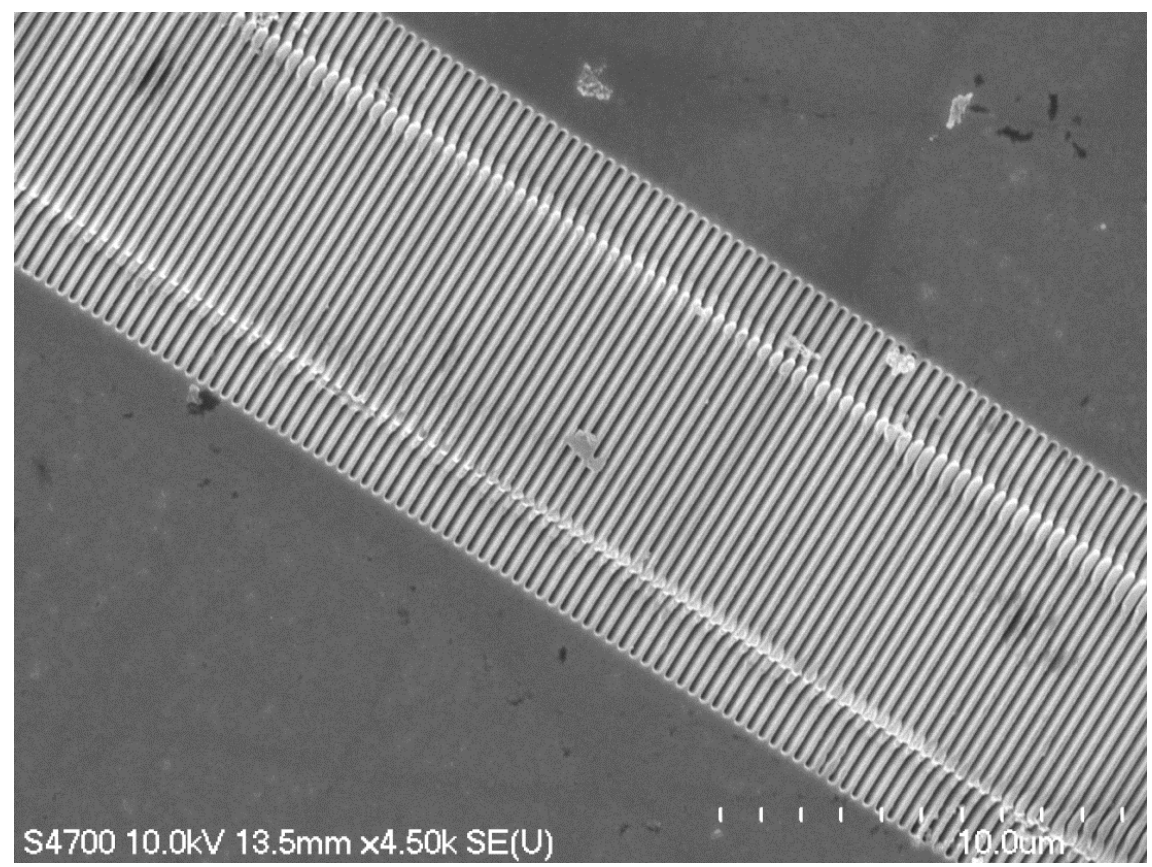

Figure 4.6 SEM micrograph of one-dimensional waveguide Bragg-filter without phase shift step.

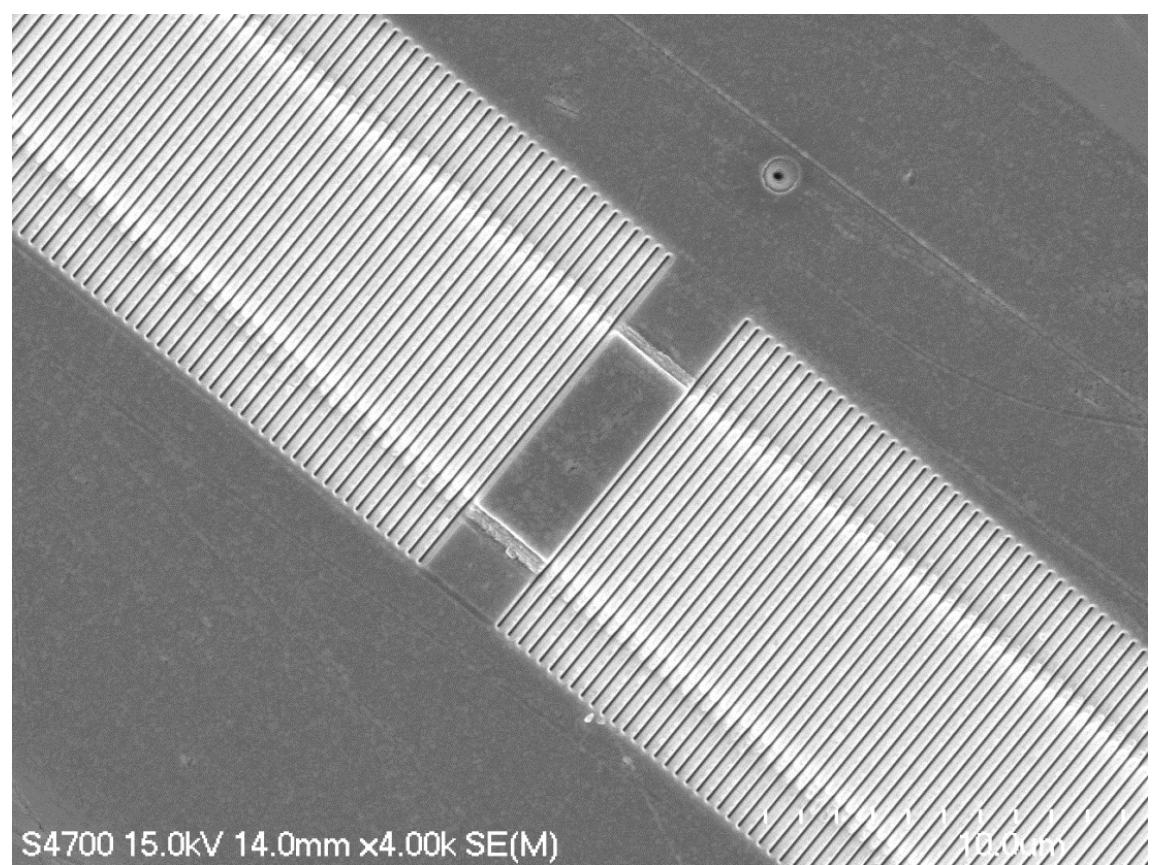

Figure 4.7 SEM micrograph of one-dimensional waveguide Bragg-filter with resonant cavity. 
The waveguides used in these experiments are single-layer structures formed on bismuth-substituted iron garnet films grown by liquid phase epitaxy on gadolinium gallium garnet (GGG) substrates. Three sets of samples of different composition were investigated. Ridge waveguides were prepared on these samples by optical lithography and plasma etching techniques. $200 \mu \mathrm{m}$-long Bragg reflectors were patterned on the ridges by focused ion beam (FIB) milling as shown in Fig.4.6 and Fig. 4.7.

A $2.86 \mu \mathrm{m}$-thick $(\mathrm{Bi}, \mathrm{Lu})_{2.8} \mathrm{Fe}_{4.7} \mathrm{O}_{12.1}$ film served as a platform for $200 \mu \mathrm{m}$ long gratings of period $(\Lambda) 338 \mathrm{~nm}$ with a single phase-shift step of $3.5 \Lambda$, denoted here as $A$. The second set, denoted as set $\mathrm{B}$, consists of a grating of period $335 \mathrm{~nm}$ with no phase shift step fabricated on $2.7 \mu$-thick $\mathrm{Bi}_{0.8} \mathrm{Gd}_{0.2} \mathrm{Lu}_{2.0} \mathrm{Fe}_{5} \mathrm{O}_{12}$ films. The film composition of the third set, denoted as set $\mathrm{C}$, is $(\mathrm{Bi}, \mathrm{Lu}, \mathrm{Nd})_{3}(\mathrm{Fe}, \mathrm{Ga}, \mathrm{Al})_{5} \mathrm{O}_{12}$ with a thickness of 1.8 $\mu \mathrm{m}$. A phase shift step of $10.5 \Lambda$ is fabricated in the middle of the grating. The grating period is $346 \mathrm{~nm}$. Refractive index data for input inplane polarization (TE) and linear birefringence between in-plane-polarized coupled light and normal-to-the planemeasured at $1543 \mathrm{~nm}$ using a prism coupler is listed in Tables 4-2, 4-3 and 4-4. The estimated accuracy in the birefringence data is \pm 0.0005 . Specific Faraday rotation for samples $\mathrm{A}, \mathrm{B}$ and $\mathrm{C}$ are $137 \% \mathrm{~mm}, 100^{\circ} / \mathrm{mm}$ and $80 \% \mathrm{~mm}$ respectively. The figures 4.8 , 4.9 and 4.10 show both experimental and computational band structures and polarization rotation for samples $\mathrm{A}, \mathrm{B}$ and $\mathrm{C}$ respectively.

Table 4-2. Refractive index and birefringence data for sample $A$

\begin{tabular}{|l|l|l|l|}
\hline & $\begin{array}{l}\text { Fundamental } \\
\text { mode }\end{array}$ & $\begin{array}{l}1^{\text {st }} \\
\text { mode }\end{array}$ & $\begin{array}{l}2^{\text {nd }} \text { order } \\
\text { mode }\end{array}$ \\
\hline $\begin{array}{l}\text { TE } \\
\text { polarization }\end{array}$ & 2.318 & 2.280 & 2.214 \\
\hline Birefringence & 0.0006 & 0.0046 & 0.0106 \\
\hline
\end{tabular}


Table 4-3. Refractive index and birefringence data for sample B

\begin{tabular}{|c|c|c|c|}
\hline & $\begin{array}{l}\text { Fundamental } \\
\text { mode }\end{array}$ & $\begin{array}{l}1^{\text {st }} \text { order } \\
\text { mode }\end{array}$ & $\begin{array}{l}2^{\text {nd }} \text { order } \\
\text { mode }\end{array}$ \\
\hline $\begin{array}{l}\text { TE } \\
\text { polarization }\end{array}$ & 2.3036 & 2.2605 & 2.1878 \\
\hline Birefringence & -0.0005 & 0.0036 & 0.0103 \\
\hline
\end{tabular}

Table 4-4. Refractive index and birefringence data for sample $\mathrm{C}$

\begin{tabular}{|l|l|l|l|}
\hline & $\begin{array}{l}\text { Fundamental } \\
\text { mode }\end{array}$ & $\begin{array}{l}1^{\text {st }} \text { order } \\
\text { mode }\end{array}$ & $\begin{array}{l}2^{\text {nd }} \text { order } \\
\text { mode }\end{array}$ \\
\hline $\begin{array}{l}\text { TE } \\
\text { polarization }\end{array}$ & 2.2460 & 2.1621 & 2.0209 \\
\hline Birefringence & 0.0024 & 0.0159 & 0.0312 \\
\hline
\end{tabular}




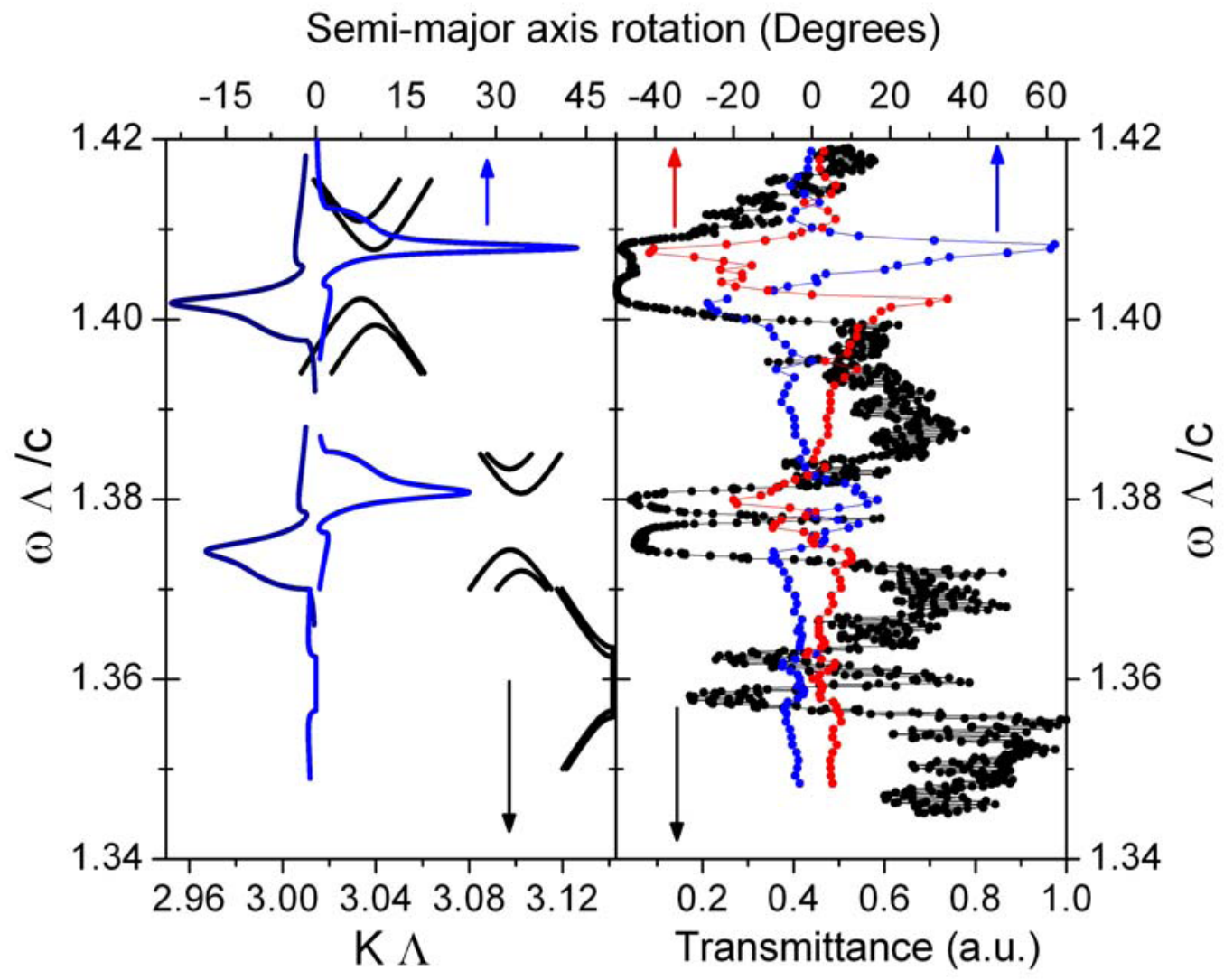

Figure 4.8 The figure plots the measured transmittance and polarization response [right panel], and calculated band structure and polarization rotation [left panel] of a one-dimensional photonic crystal with phase shift step of $3.5 \Lambda$ patterned on a $2.86 \mu \mathrm{m}$-thick $(\mathrm{Bi}, \mathrm{Lu})_{2.8} \mathrm{Fe}_{4.7} \mathrm{O}_{12.1}$ film (sample set A). Separate curves for the calculated semi-major axis orientation correspond to different Bloch states. The red and blue data points on the right panel describe the orientation of the semi-major axis of the polarization ellipse for opposite magnetizations collinear with the ridge waveguide axis. 


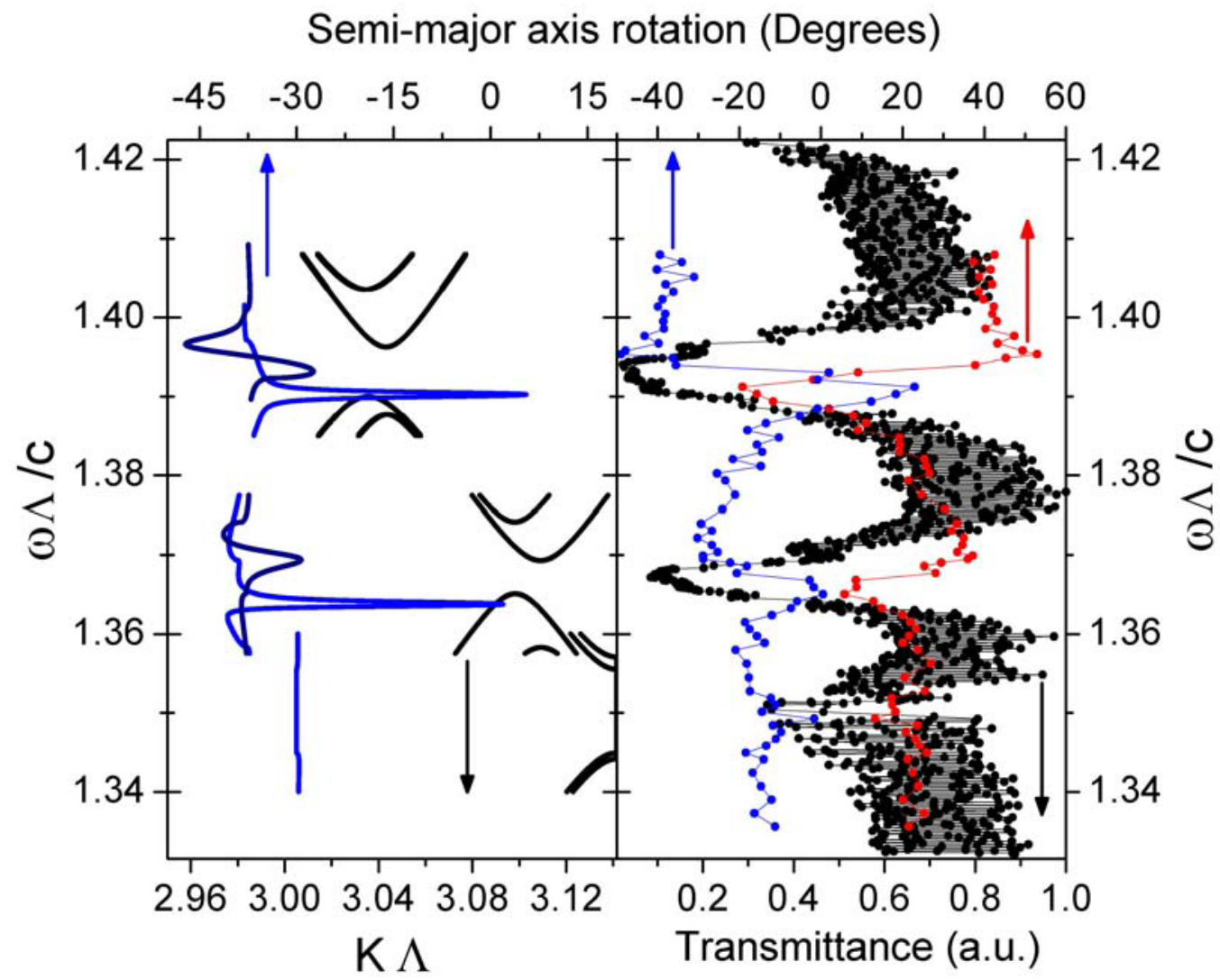

Figure 4.9 Measured transmittance and polarization response [right panel], and calculated band structure and polarization rotation [left panel] of a onedimensional Bragg filter without phase shift step patterned on a $2.7 \mu \mathrm{m}$-thick $\mathrm{Bi}_{0.8} \mathrm{Gd}_{0.2} \mathrm{Lu}_{2.0} \mathrm{Fe}_{5} \mathrm{O}_{12}$ film (sample set $\mathrm{B}$ ). Separate curves for the calculated semimajor axis orientation correspond to different Bloch states. The red and blue data points on the right describe the orientation of the semi-major axis of the output polarization ellipse for opposite magnetizations collinear with the ridge waveguide axis. 


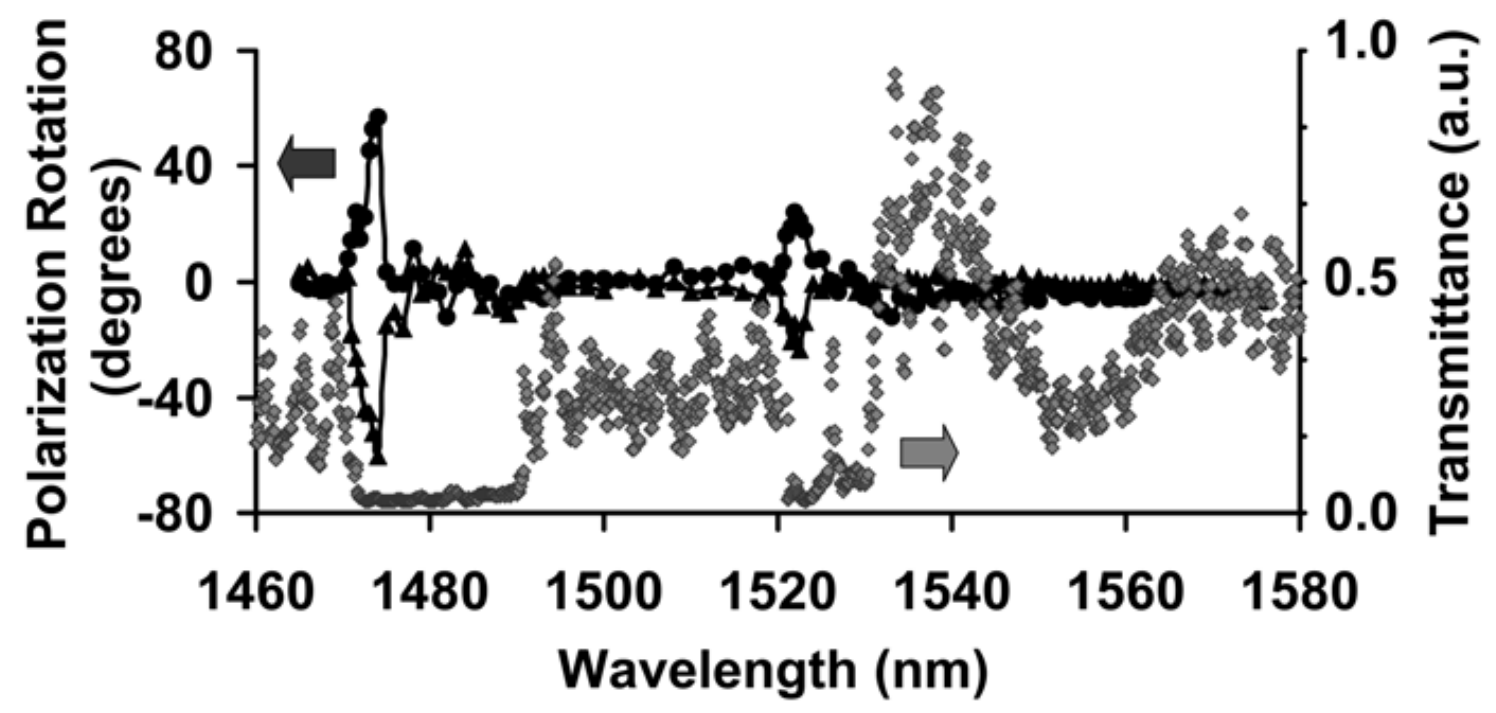

Figure 4.10 Measured transmittance and polarization response for a onedimensional photonic crystal with a single phase shift step patterned on a $(\mathrm{Bi}, \mathrm{Lu}, \mathrm{Nd})_{3}(\mathrm{Fe}, \mathrm{Ga}, \mathrm{Al})_{5} \mathrm{O}_{12}$ film of thickness $1.8 \mu \mathrm{m}$. Black triangles and solid circles plot the orientation of the semi-major axis of the output polarization ellipse relative to the linear input polarization for opposite magnetization directions collinear with the ridge waveguide axis.

Transmittance and polarization rotation output of the Bragg waveguides were measured for linearly polarized TE inputs coupled into the waveguide facets. Measurements were taken under a saturation magnetic field of 3000 e parallel to the waveguide axis in the forward and backward directions. The transmittance spectrum is the energy output of the electromagnetic wave with all polarization directions. Polarization rotations were measured by the motorized rotating polarizer. Here the polarization rotation is defined as the angle between the linear input polarization and the semi-major axis of the output polarization. The band gaps are formed following the Bragg scattering condition for mode coupling given by $\lambda=\left(n_{f}+n_{b}\right) \Lambda$ with different stop bands corresponding to forward fundamental to different backscattered higher order modes. These band gaps yield large polarization rotations, especially near the band edges that grow with increasing backscattered-mode order as shown in Figs. 4.8-4.10. The ellipticity of the 
output light defined as the ratio of semi-minor to semi-major axes lengths, are plotted in Fig 4.11. An interesting feature here is that large polarization rotations are observed even in the presence of large linear birefringence. Generally, linear birefringence suppresses the Faraday rotation.

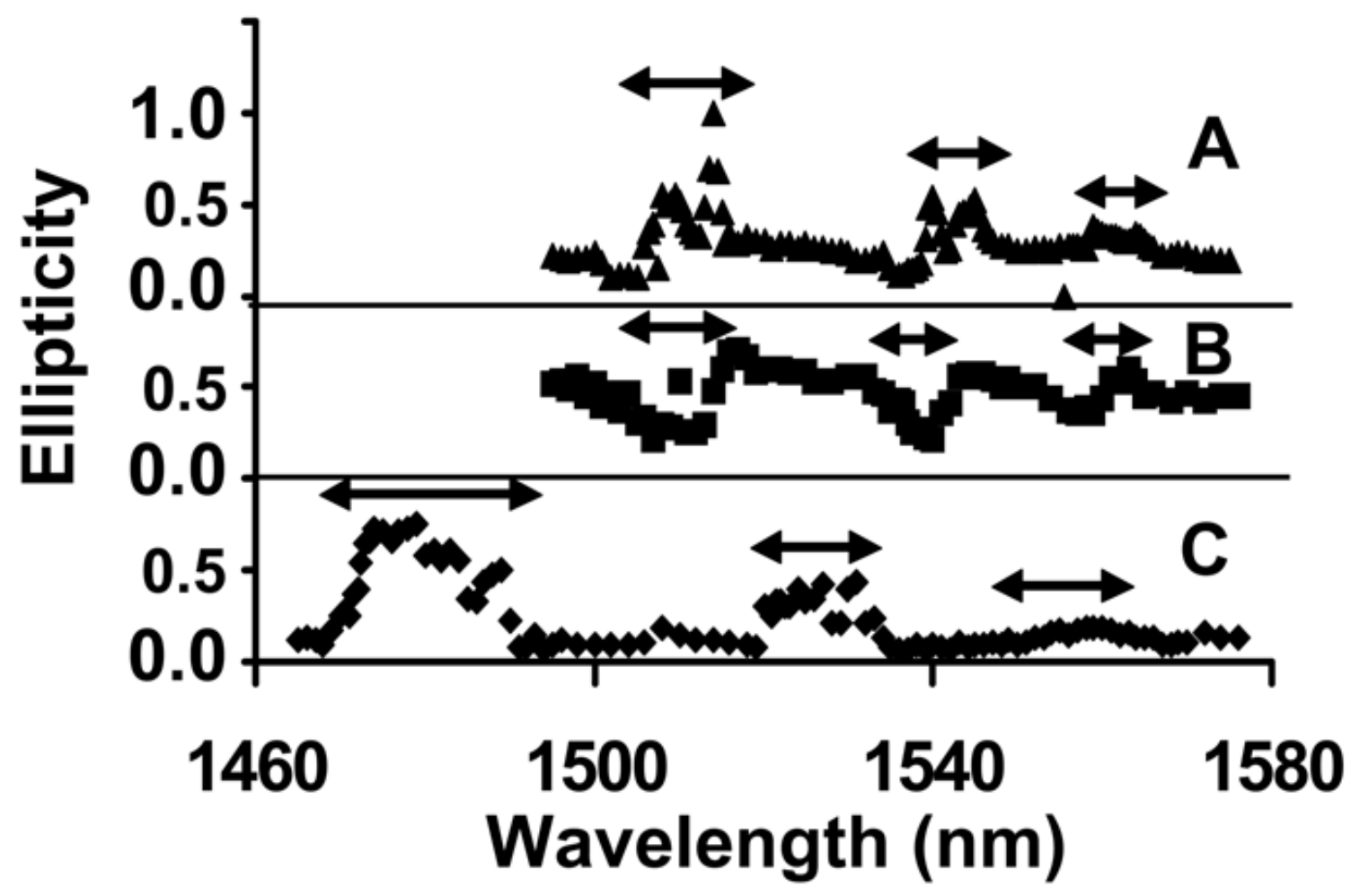

Figure 4.11 Measured ellipticity in the output polarization for Bragg filters patterned on $(\mathrm{Bi}, \mathrm{Lu})_{2.8} \mathrm{Fe}_{4.7} \mathrm{O}_{12.1}$ film (sample set $\mathrm{A}$ ), $\mathrm{Bi}_{0.8} \mathrm{Gd}_{0.2} \mathrm{Lu}_{2.0} \mathrm{Fe}_{5} \mathrm{O}_{12}$ film (sample set $\mathrm{B}$ ) and $(\mathrm{Bi}, \mathrm{Lu}, \mathrm{Nd})_{3}(\mathrm{Fe}, \mathrm{Ga}, \mathrm{Al})_{5} \mathrm{O}_{12}$ film (samples set $\mathrm{C}$ ). Ellipticity is defined as the ratio of the semi-minor to semi-major axes of the polarization ellipse in the optical electric field amplitude. The horizontal double-tipped arrows indicate the locations of the stopbands in each case.

Figures 4.8 and 4.9 (left panel) plot the calculated band structure for the stack model system with local normal modes of different orders travelling in opposite directions. Two nearly overlapping gyrotropic bandgaps corresponding to different Bloch states are 
visible for high-order mode backscattering. The band structures are calculated considering the material and the structure parameters of their experimental counterpart. Deviations up to $2 \%$ in modal refractive indices from the measured slab waveguide data, with $0.1 \%$ maximum grid size are allowed in order to match stop band bandwidths, center-wavelengths and polarization rotations. The calculated band structure is in good agreement with the experimental data, with Bloch modes exhibiting higher rotations at the band edges, a similar trend as in experimental results. The experimental data in Figs. 4.9 (sample B), show large polarization rotations for a Bragg grating without resonant cavity, where no photon trapping acts to increase the optical path length. Also, there is no significant rotation seen at the resonant wavelengths for the samples (A and C) with resonant cavities. Therefore, the increased rotation is not due to photon trapping but a different mechanism characteristic to magneto-optic Bragg waveguides.

The increased rotation is due to the back-reflection of selective polarization components out of the incident fundamental waveguide modes. In other words, the enhanced polarization rotation at the stop band edges originates from the partial selective reflection of positive- and negative-helicity higher order elliptical normal modes. Opposite helicity modes possess spectrally-detuned stop bands and orthogonally-oriented elliptical polarization. This detuning is due to the large birefringence of the high-order backscattered modes, resulting in rotated transmitted polarization near the band edges. 


\subsection{Sensor design}

The optical sensor presented here is designed based on the large polarization rotational response to changes in cover refractive index in magneto-optic birefringent waveguide photonic crystals. The theoretical model discussed in the last section predicts that the polarization rotation is very sensitive to variations in refractive index or linear birefringence in the film, substrate, or cover layer. Figure 4.12 plots the output signal polarization rotation versus refractive index modeled for a typical $(\mathrm{Bi}, \mathrm{Y}, \mathrm{Lu})_{3}(\mathrm{Fe}$, $\mathrm{Ga})_{5} \mathrm{O}_{12}$ magneto-photonic crystal structure with realistic cover and waveguide indices and film thickness. A change in the waveguide mode indices induced by the change in cover index alters the characteristics of the propagating Bloch modes in waveguide photonic crystal resulting changes in output polarization rotation. A steep polarization change versus index occurs in the 2.175 to 2.176 modal-refractive-index range for this particular case. Therefore, the sensor design is aimed for detection in 1.3-1.6 refractive index range. The theoretically predicted strong polarization rotation sensitivity near the band edges was experimentally observed during the initial tests as shown in Fig. 4.13. These tests were performed by adding and removing vacuum grease (cover layer) on top of the photonic crystal. Polarization changes as large as $80^{\circ}$ were observed. Hence, it is evident that this novel sensing technique delivers significant sensitivity and a fairly wide sensing window. The sensor calibration was done by applying refractive index liquids of known refractive indices on the photonic crystal and measuring the output polarization rotation. Next, the sensing technique was developed for detection of toxic substances by employing analyte sensitive polymer cover layers in the photonic crystal. The refractive index changes induced by analyte adsorption into a polymeric layer deposited on the photonic crystal surface (cover layer in Fig. 4.14) affect the polarization rotation of the output light. The proceeding sub sections present the experimental and analytical results of sensor characterization and development of the technique for aqueous ammonia and methanol detection. 


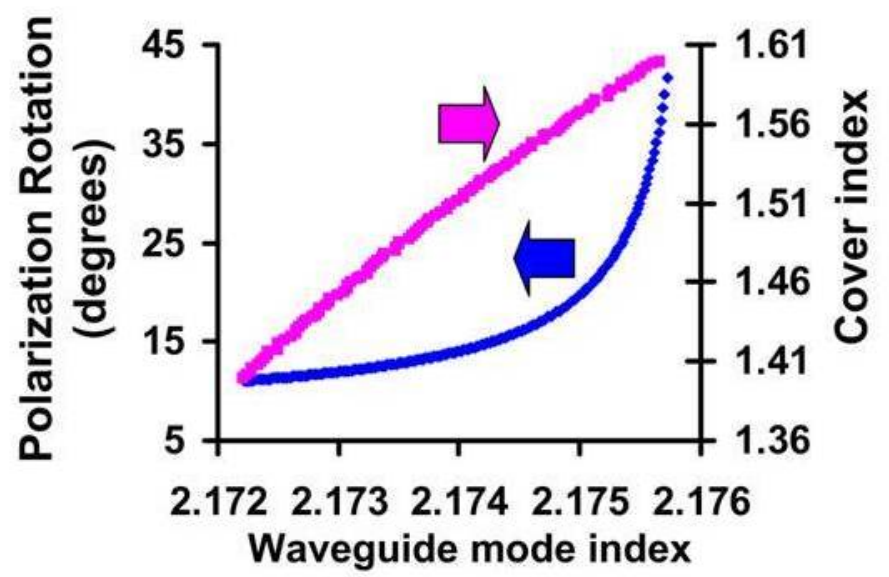

Figure 4.12 Angular dependence on index of the semi-major axis of the output polarization ellipse in the overlap pass band formed near the gyrotropic degenerate band gap.

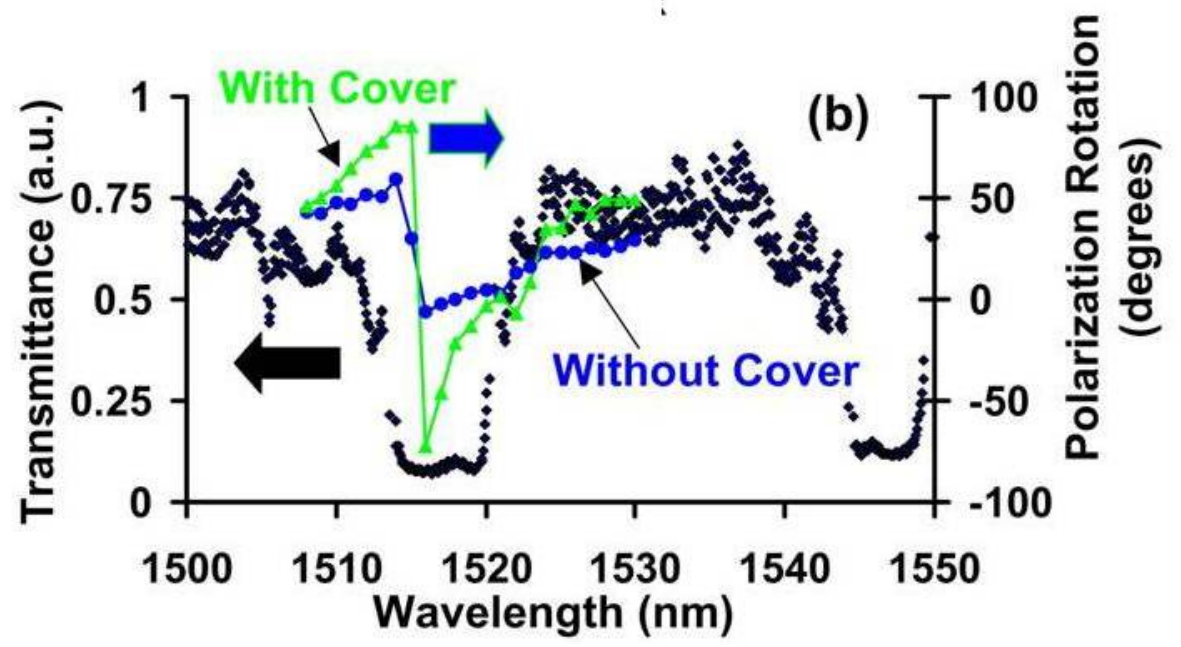

Figure 4.13 Initial sensor testing results for air cover and vacuum grease cover. 


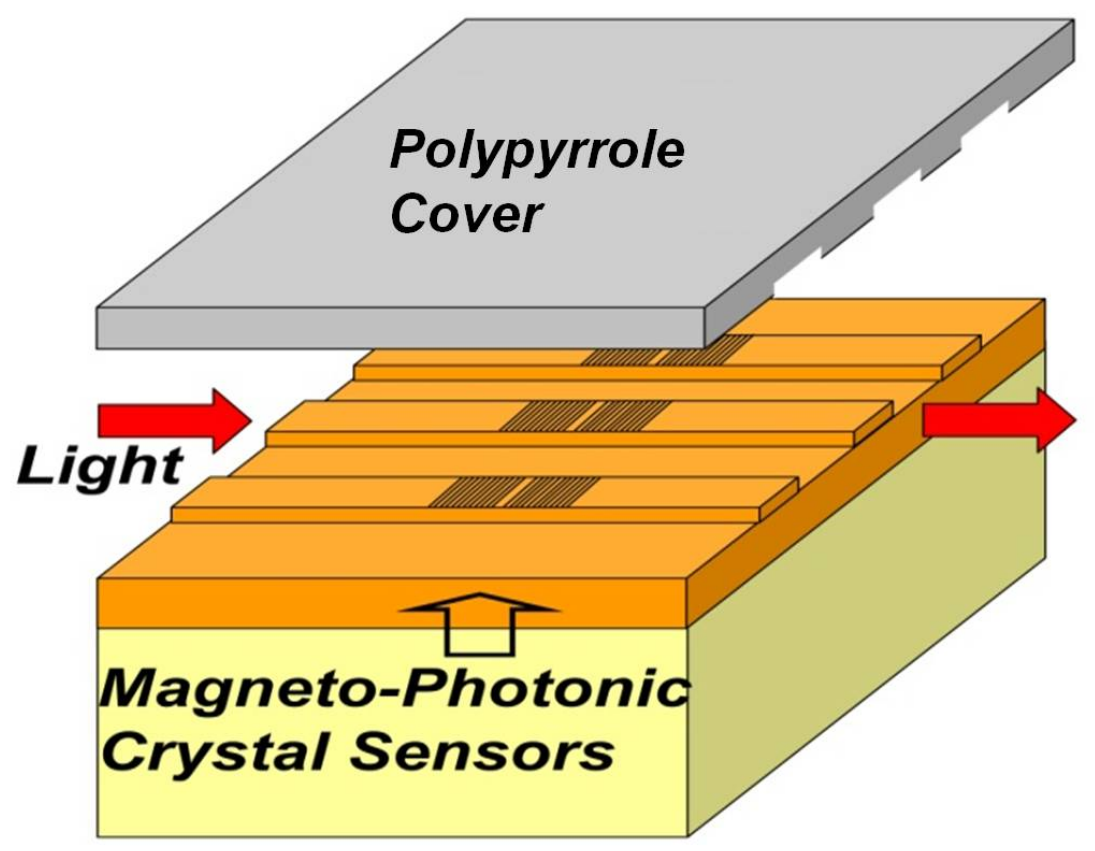

Figure 4.14 schematic representation of magneto-photonic crystal sensor with Polypyrrole cover layer

\subsubsection{Sensor characterization}

A systematic sensor characterization was necessary in order to study the performance and the sensor sensitivity. Three samples were optically tested by modifying the cover refractive index with known refractive index liquids.

\subsubsection{Sample information}

Commercially grown liquid-phase-epitaxial mono-crystal garnet films on (100) gadolinium gallium garnet (GGG) substrates were used for sample preparation. All three samples have the same film thickness of $2.7 \mu \mathrm{m}$ and composition of $\mathrm{Bi}_{0.8} \mathrm{Gd}_{0.2} \mathrm{Lu}_{2.0} \mathrm{Fe}_{5} \mathrm{O}_{12}$. Standard photolithography and plasma etching were utilized to pattern $6 \mu \mathrm{m}$ wide $600 \mathrm{~nm}$ ridge-height waveguide structures on the film. One dimensional photonic band gap structures (Bragg gratings) were fabricated onto the ridge waveguide by ion beam milling in a Hitachi FB-2000A focused ion beam system. 
A $200 \mu \mathrm{m}$-long Bragg grating with period $337 \mathrm{~nm}$ and groove-depth $700 \mathrm{~nm}$ is denoted as Sample 1. The ridge waveguide is $0.9 \mathrm{~mm}$ long, and waveguide mode refractive indices of the film before surface-patterning measured on the film slab using prism coupler are 2.3041, 2.2616, and 2.1896 for the fundamental, first and second order TE modes, respectively. Linear birefringence, measured for the first three modes are 0.0005 , 0.0053 and 0.0130 . The Faraday rotation per unit length in the film is $100^{\circ} / \mathrm{mm}$. Samples 2 and 3 consist of $200 \mu \mathrm{m}$ long photonic crystals with period of $348 \mathrm{~nm}$ and groove depths of $700 \mathrm{~nm}$ and $600 \mathrm{~nm}$. Their corresponding waveguide lengths are $0.8 \mathrm{~mm}$ and $1 \mathrm{~mm}$. Prism coupler measurements of refractive indices for TE inputs for fundamental, first, and second modes are 2.3030, 2.2603 and 2.1882, respectively; linear birefringence measured for the first three modes are $0.0008,0.0051$, and 0.0121 respectively. The specific Faraday rotation of this film is $80^{\circ} / \mathrm{mm}$.

\subsubsection{Refractive index liquids}

Cover indices of the waveguide photonic crystal were modified by applying a refractive index matching liquids of known indices. These refractive index liquids were purchased from Cargille Labs [96]. The bulk index measurement feature of the prism coupler was utilized to re-measure the refractive indices of the liquids at $1543 \mathrm{~nm}$ wavelength.

The refractive index liquid was brought into contact with the prism by applying a thin layer of the liquid on the prism surface. Laser light incident on the prism is refracted into the bulk material when the angle of incidence at the prism-refractive index liquid interface falls below the critical angle for the two materials. The corresponding angle can be easily measured by observing the sudden drop of light intensity at the detector. A schematic depiction of the prism coupler set up for bulk measurements and a graphical representation of intensity vs angle of incidence is shown in Fig 4.15. The refractive index of the bulk material can be calculated using equation 4.1 [97]. 


$$
\theta_{c}=\sin ^{-1} \frac{n}{n_{p}}
$$
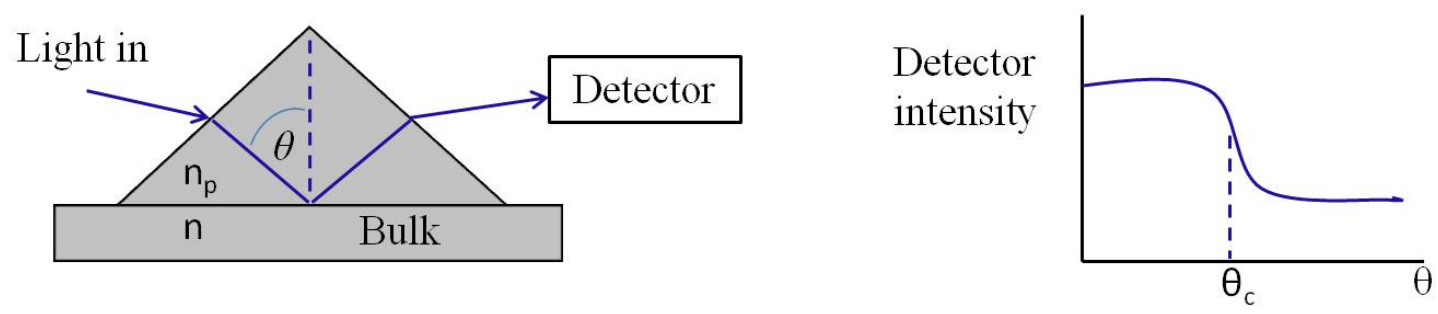

Figure 4.15Prism coupler measurement set up for bulk materials

Five liquids with a refractive index ranging from 1.31 to 1.61 were used for the sensor characterization study. The prism coupler measured index data is listed in Table 4-5.

Table 4-5. Prism coupler measured index data for refractive index liquids used in sensor characterization

\begin{tabular}{|c|c|}
\hline Refractive index liquid & Refractive index \\
\hline 1 & 1.31 \\
\hline 2 & 1.41 \\
\hline 3 & 1.48 \\
\hline 4 & 1.54 \\
\hline 5 & 1.60 \\
\hline
\end{tabular}




\subsubsection{Optical tests}

Transmittance and the polarization rotation of the test samples were measured for air cover prior to modifying the cover index of the PC by applying refractive index liquids. (Fig. 4.16) The sample was placed on a stage with $\mathrm{x}, \mathrm{y}$, and $\mathrm{z}$ controls and a saturating magnetic field (300Oe) was applied along the waveguide axis by mounting the sample on a permanent magnet. The light from a tunable laser source with wavelength range from $1480 \mathrm{~nm}$ to $1580 \mathrm{~nm}$ was coupled into the waveguide by end-fire fiber coupling using a single mode lensed fiber with diameter of about $3.3 \mu \mathrm{m}$.

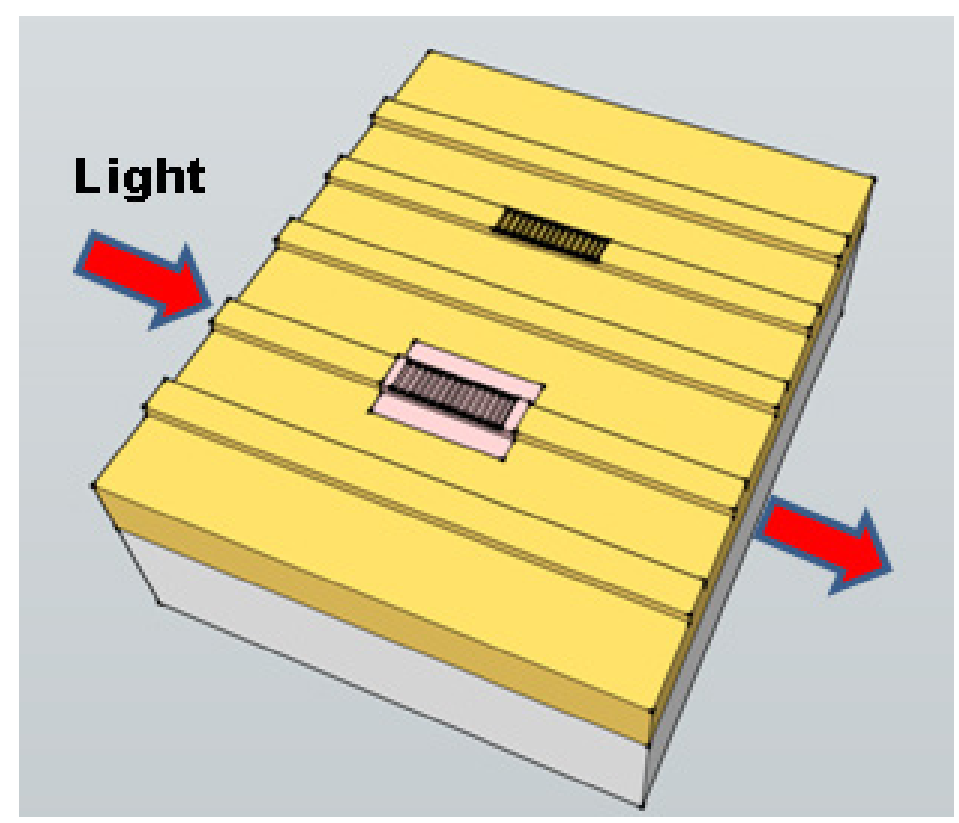

Figure 4.16 Schematic picture of hotonic crystal cover modified with refractive index liquid applied over the PC

The polarization of the input light was adjusted to TE (horizontal) polarization by a digital polarization controller (Agilent 11896A). The output beam from the waveguide passes through a 10x microscope objective and is split into two beams by a $50 \%$ nonpolarizing beam-splitter. One beam is sent to a photo detector (Newport 918D-IR-OD3 photodetector read through Newport 1931-C Power Meter) for beam intensity 
measurement and the other beam is sent to an infrared recording camera to monitor the beam profile. The transmittance from the PC waveguides was recorded through a Lab view interface which synchronizes the power meter and the tunable laser source. Two methods were employed to measure the polarization of the output light from the waveguide PC. In the first method, the output light was recorded through a motorized polarizer (Newport Universal motion controller, Model ESP100) which rotates through $360^{\circ}$ in steps of $10^{\circ} \mathrm{s}^{-1}$. The polarization rotation angle can be obtained by plotting the spatial intensity spectrum of the output light (details in Sect. 3.4.1.1). In the second method, the state of polarization of output light was determined by evaluating the Stokes parameters of the light. Stokes vectors of the output light were extracted using a combination of a polarizer and a quarter wave plate. Polarization rotation is defined here as the angle between the semi-major axis of the output polarization ellipse and the linear TE input polarization.

The refractive index liquids were applied on the PC using a thin optical fiber attached to a micro manipulator. The transmittance and the polarization rotation of the PC output were measured for each modified cover. Figures 4.17, 4.18 and 4.19 show the measured transmittance for air cover and polarization rotation at modified cover for sample 1, sample 2 and sample 3, respectively. These measurements were taken for the stop band formed by the coupling of forward-propagating fundamental and backscattered second-order modes. After each successive refractive index measurement the samples were cleaned with a cleaning solvent and re-tested. Re-tests with air as cover were found to reproduce those prior to index liquid application. A bandwidth average of the rotation was taken in order to reduce experimental measurement fluctuations and to fold in changes in the spectral shape of the response with cover index. 


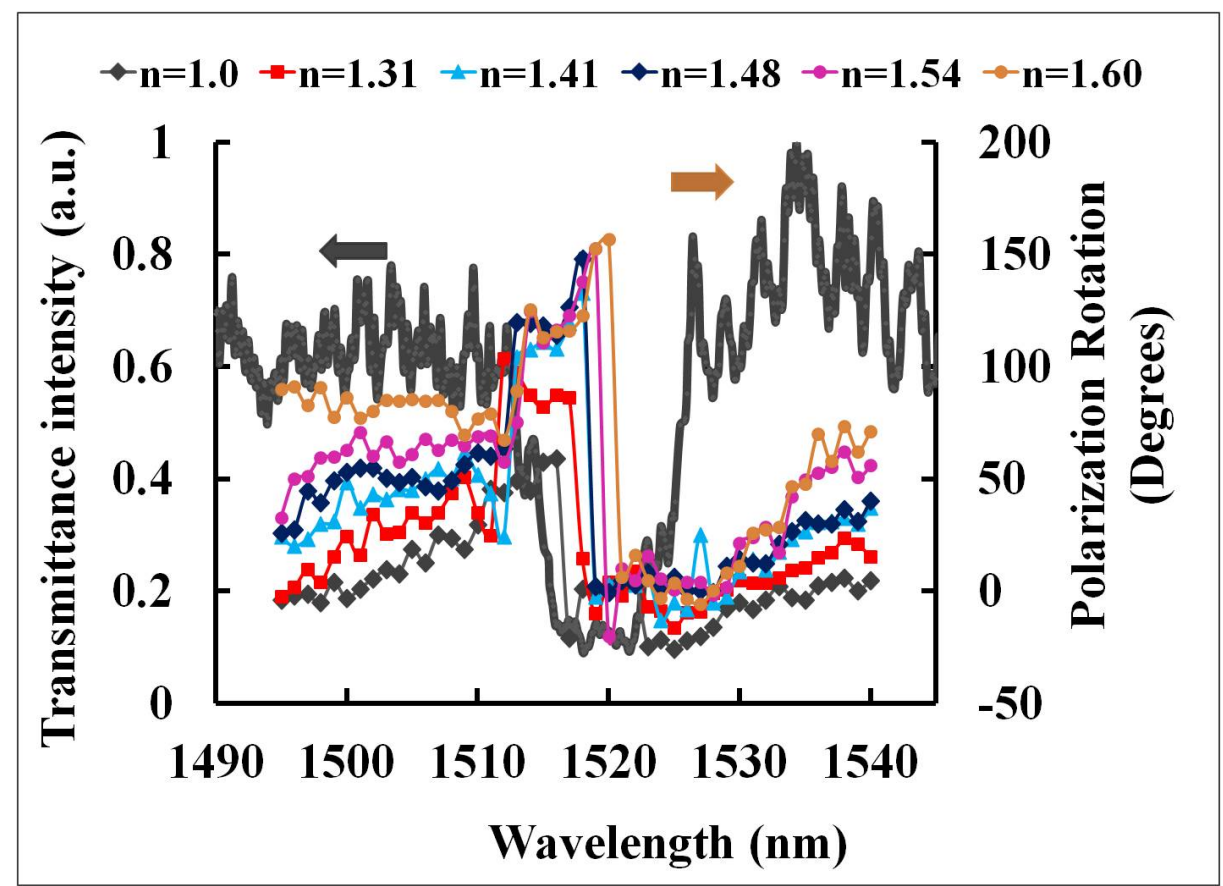

Figure 4.17 Measured transmittance and polarization rotation for varying cover index of the waveguide photonic crystal for sample 1.

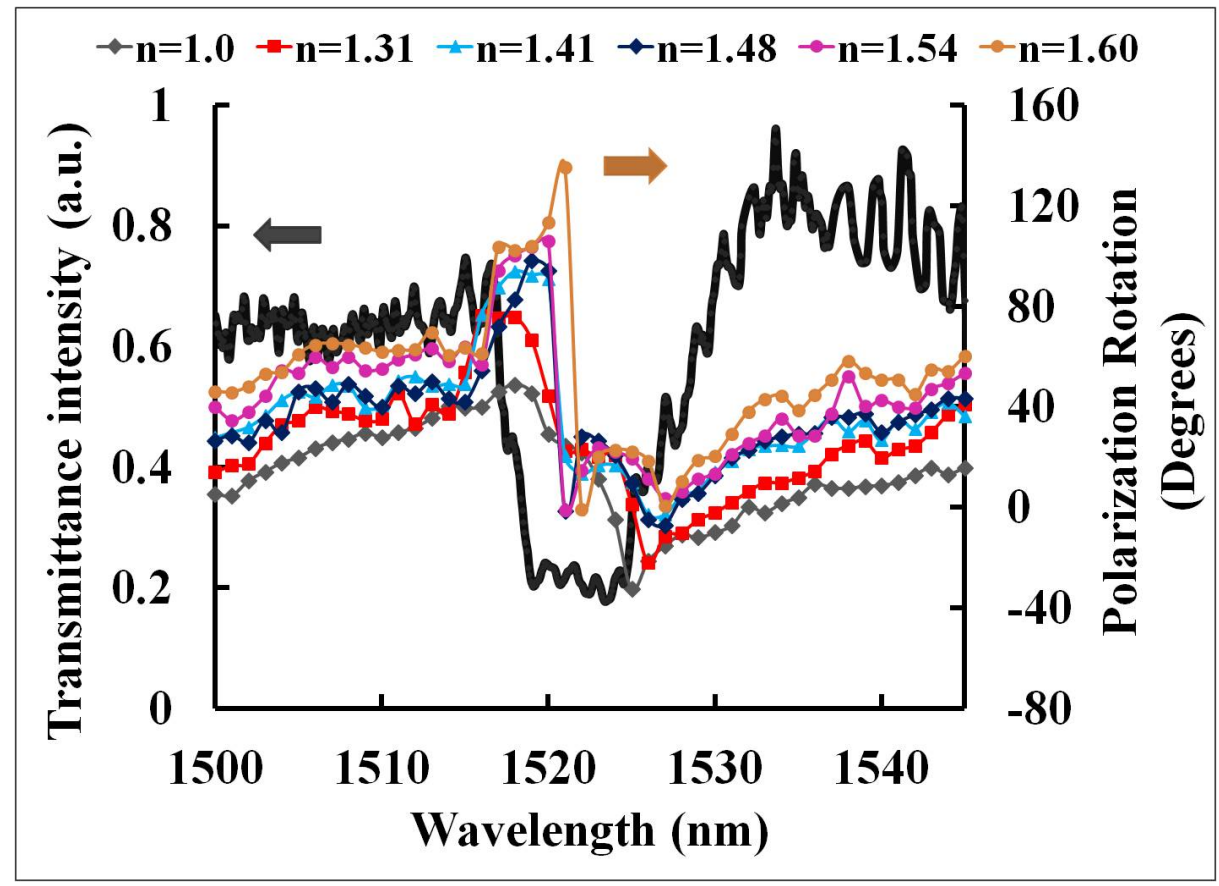

Figure 4.18 Measured transmittance and polarization rotation for varying cover index of the waveguide photonic crystal for sample 2. 


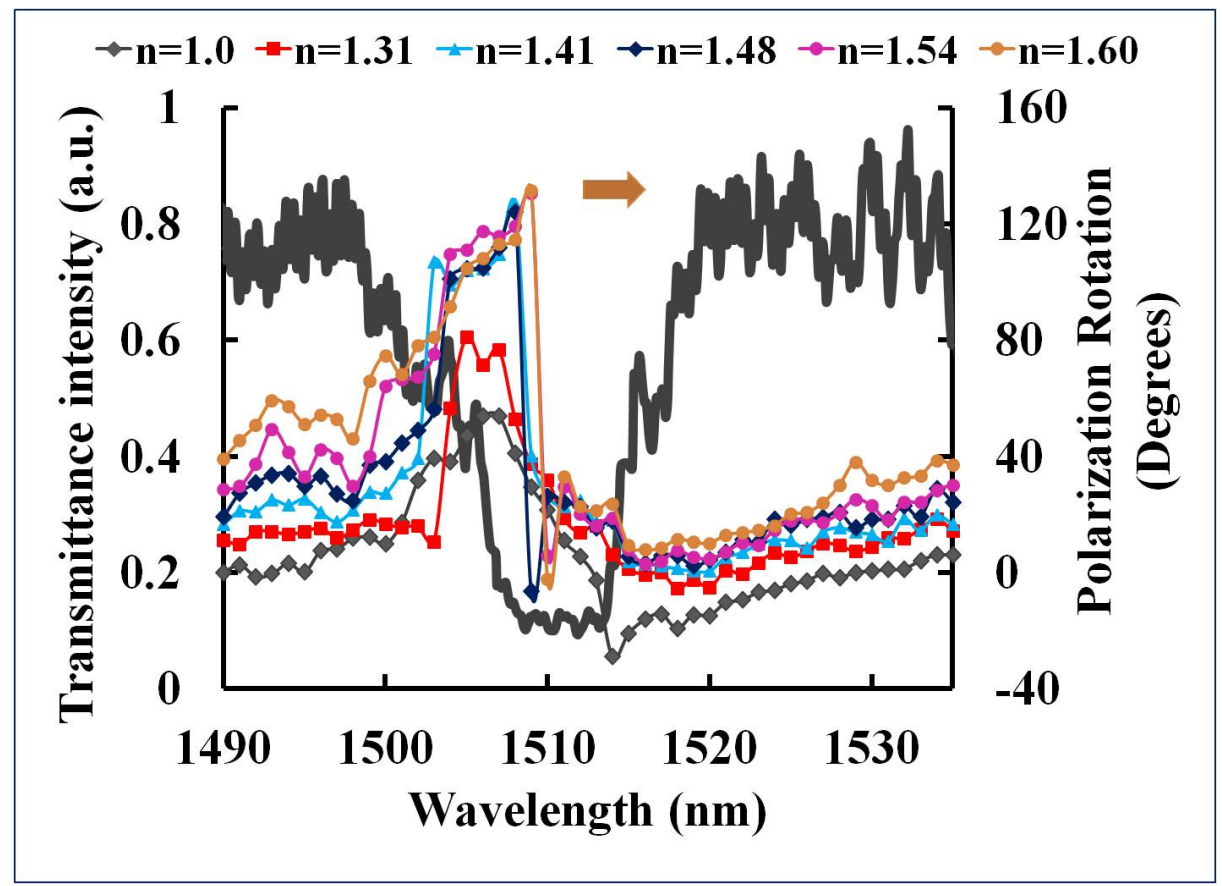

Figure 4.19 Measured transmittance and polarization rotation for varying cover index of the waveguide photonic crystal for sample 3.

Figure 4.20 shows the average polarization rotation with respect to cover refractive index for all three samples. The average polarization rotation was calculated by integrating the rotation over the entire bandwidth of the stop band and vicinity, using the polarization outside the stop band as base line. The base line varies with cover index because of the rotation outside the grating structure. Defining a base line this way precludes folding in the rotation accrued in the waveguide before and after the photonic crystal structure. In this way the end result is purely due to the gyrotropic band gap effect. Differences in measured rotational strength are believed to result from grating groove-depth differences between the samples. 


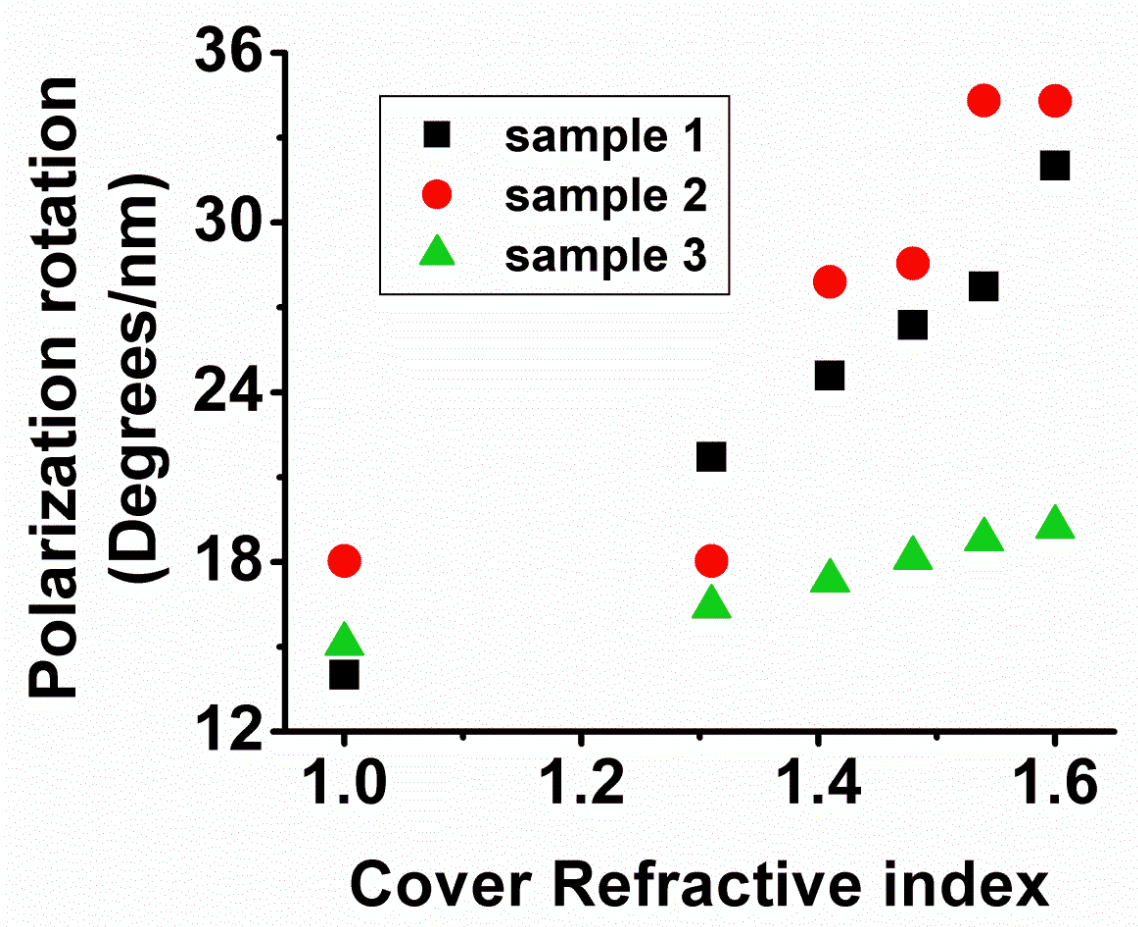

Figure 4.20Average polarization rotation measured for samples 1, 2 and 3.

\subsubsection{Theoretical simulation}

The theoretical model (Stack model) presented in Sect. 2.3.5 is used for theoretical calculations of the orientation of the Bloch modes (semi-major axis) with respect to the change in cover index. The model is optimized by switching off the gyrotropy parameter $g$ and matching the center-wavelength and bandwidth of the model to experimentally-determined transverse-electric (TE) and transverse magnetic (TM) stop bands. The gyrotropy is then turned back on and the stop bands and Bloch mode orientations are recalculated. Modified waveguide mode refractive indices due to change in cover index were introduced to the model for each cover index. The modified waveguide mode indices were obtained through beam propagation simulations by introducing corresponding cover indices in the ridge waveguide structure. The 
calculated band structure for fundamental to second-order-waveguide mode coupling for air cover index and orientation of one of the Bloch modes as a function of cover index are shown in Fig. 4.21.

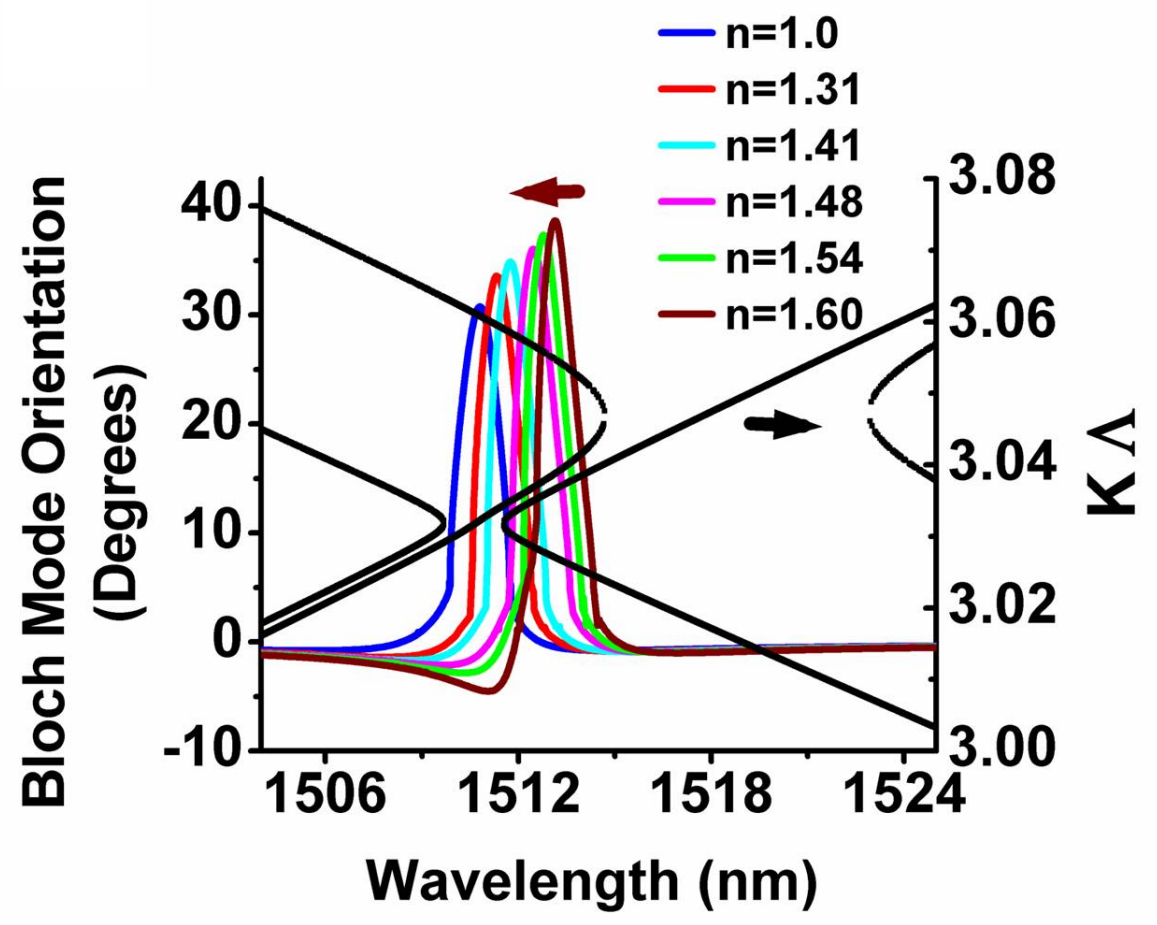

Figure 4.21 Calculated band structure for a bilayer stack with period $\Lambda$ and computed orientation of the semi-major axis of one of the Bloch modes versus cover index.

Here the Bloch wave vector $\mathrm{K}=2 \pi / \Lambda$, where $\Lambda$ is the grating period. The model shows not only a large enhancement in Bloch-mode polarization rotation (direction of semimajor axis) near the band edge but also a strong sensitivity of mode rotation to small refractive index changes in the waveguide cover index. Similar to the experimental case, the mode rotation is averaged over the main peak bandwidth of the mode and presented in Fig. 4.22. The orientation of all the Bloch modes shows a similar increasing trend with increasing cover index. 


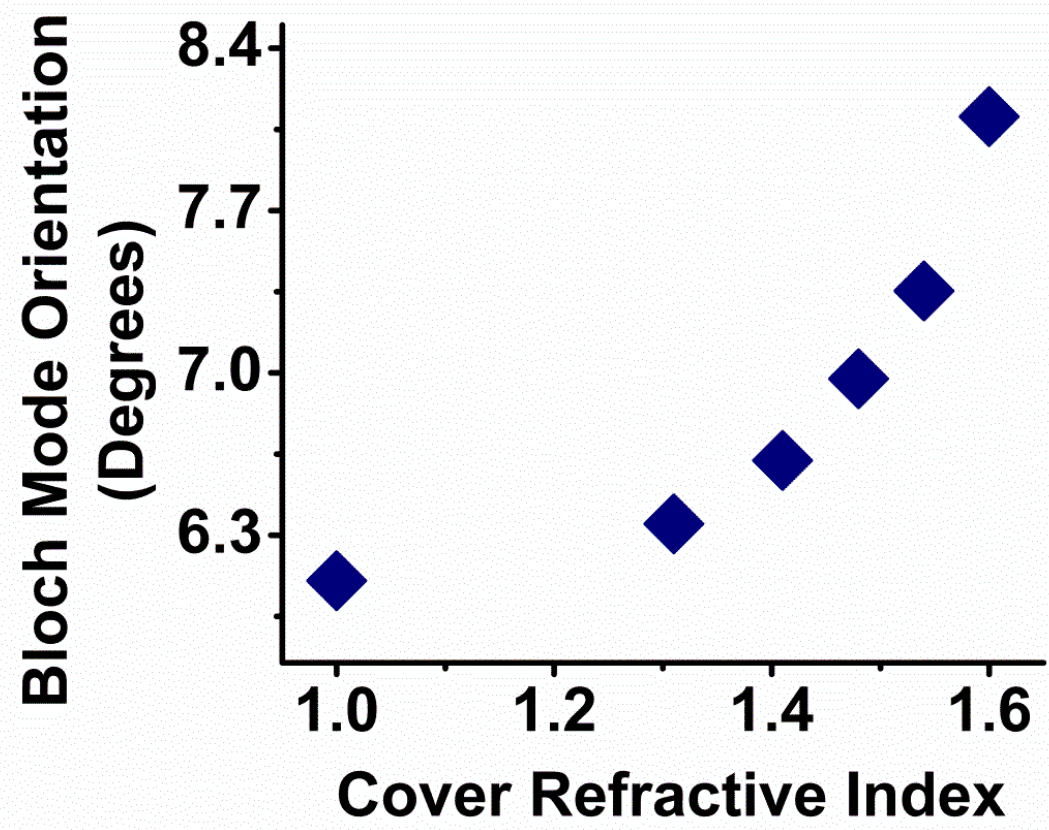

Figure 4.22 Bloch mode polarization averaged over the band width rotation for increasing cover index

\subsubsection{Photonic crystal and plain waveguide polarization rotation comparison}

The polarization rotation of a plain waveguide (no Bragg grating) with the same cover indices was measured for comparison with that for a photonic crystal structure. Figure 4.23 shows the transmittance of a plane waveguide for air cover and polarization rotation for varying cover indices. The material and dimensional parameters of waveguide were exactly the same as in sample 1. The magnitude of the polarization rotation for plane waveguide is significantly lower than inside the band gap of the photonic crystal at higher indices. 


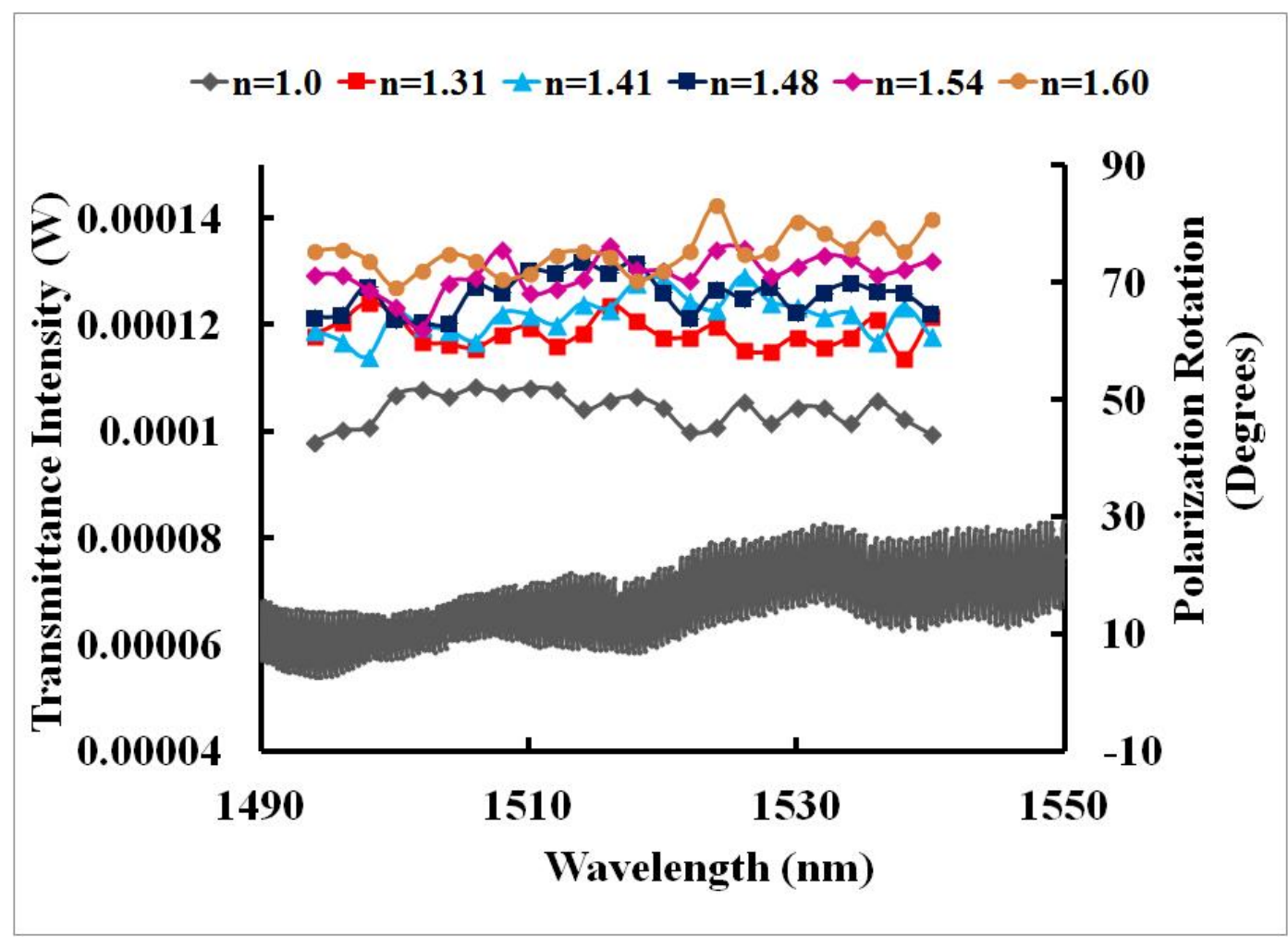

Figure 4.23 Transmittance and polarization rotation measured for a plain waveguide.

The average polarization rotation of the plane waveguide over the same band width is then compared with the average polarization rotation of the waveguide PC and shown in Fig. 4.24. In order to be able to compare both types of rotations directly, the same baseline was used for both, namely, that of the input polarization, or zero rotation (TE mode). The comparison shows a faster rate of change and stronger sensitivity in the photonic crystal structure. Hence, the gyrotropic band gaps formed by counter propagating waveguide modes play an important role in the observed enhanced polarization rotation in the magneto-optic PC structures. The strength of the sensor response is dictated by its sensitivity to changes in the refractive index of the cover layer. As such, the presence of birefringence, in addition to the Faraday rotation, plays a positive role in the sensor response. Generally, linear birefringence partially suppresses the Faraday rotation in a magneto-optic medium. 


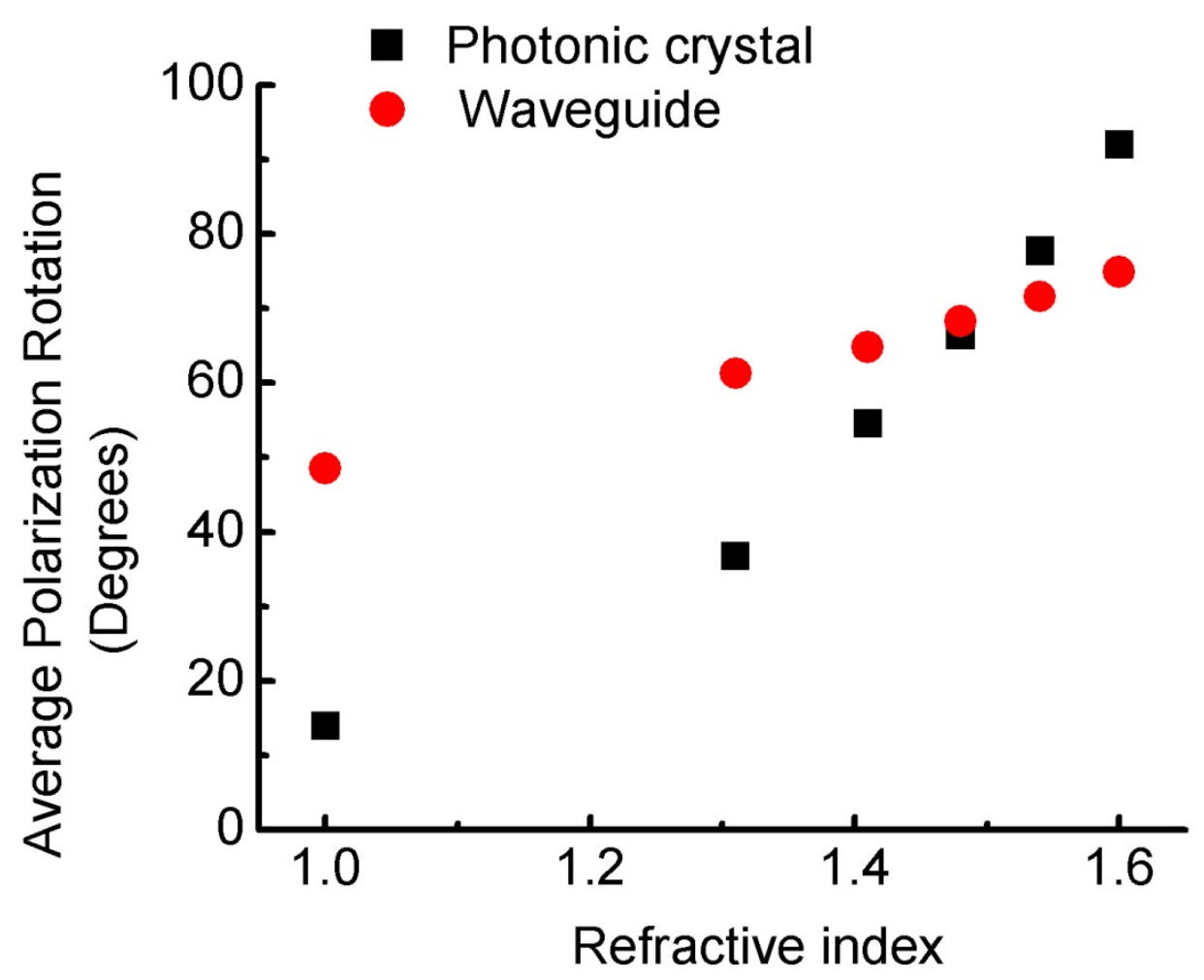

Figure 4.24 Average polarization rotation versus cover index for a waveguide photonic crystal and for a plain waveguide.

However, the increase in cover index from that of air to larger indices lessens this suppression by reducing the index contrast between cover and substrate. In other words, the geometrical birefringence caused by the difference in refractive index between cover and substrate is reduced by an increase in cover index, leading to changes in the output polarization. Thus, the combined presence of linear birefringence and Faraday rotation plays a useful role by contributing to the polarization rotation change with cover index.

The normal modes in the photonic crystal are no longer circularly-polarized modes as in a Faraday rotator, but are elliptically-polarized Bloch modes. Spectral dispersion and polarization state changes of these modes in and near the band gap contribute to the 
net polarization rotation detected at the output of the device. The spectral polarization state dispersion is strongly affected by the photonic crystal near the band gap edges. This spectral dispersion by the photonic crystal structure and its effect on Bloch mode polarization lead to an enhanced sensitivity to cover index changes over a plain waveguide.

\subsubsection{Sensor sensitivity}

Sensor sensitivity $(\mathrm{S})$ is one of the very important parameters in a sensing device which characterizes the device. Also evaluation of the sensitivity is important when it comes to comparison with other sensing techniques. Sensitivity is the magnitude of sensor transduction signal change in response to the change in analyte [1]. Sensitivity of a device is highly dependent on the strength of light matter interaction.

The sensor sensitivity of the magneto-photonic crystal sensor can be estimated by taking the ratio of change in polarization rotation to change in refractive index, given below.

$$
D=\frac{\text { Change in polarization rotation }}{\text { Change in cover refractive index }}=\frac{\Delta \theta^{\circ}}{\Delta n}
$$

The estimated sensitivity for our devices is $71^{\circ} \mathrm{RIU}^{-1}$ in the higher (most sensitive) index range, where RIU is the refractive index unit.

The performance of RI based sensors can be compared by their RI sensitivity or the detection limit (DL). The detection limit of a sensing device is determined by taking in to account the factors such as RI sensitivity, system resolution, and a number of noise sources and given by the following equation,

$$
D L=\frac{R}{S}
$$

where $\mathrm{R}$ is the resolution, the smallest measurable quantity of the detection signal in the sensing device [98]. The detection limit of our sensors is estimated to be $\sim 10^{-3}$ RIU. DL 
gives a rough quantitative estimate of the sensing performance of the device under consideration. Therefore DL could be used as a measure when comparing the sensing performance with other optical sensors flat forms and designs. Demonstrated detection limit for various RI sensors are listed in Table 4-6.

Table 4-6. Detection limit of various RI sensing technologies.

\begin{tabular}{|l|c|}
\hline \multicolumn{1}{|c|}{ RI sensor configuration } & Detection limit (RIU) \\
\hline 1-D PC [100] & $10^{-6}$ \\
\hline 2-D PC microcavity [101,102] & $\sim 10^{-3}$ \\
\hline PC fiber[7] & $10^{-4}$ \\
\hline Long period grating[103] & $10^{-5}$ \\
\hline Nano fiber[104] & $10^{-7}$ \\
\hline Surface plasmon resonance[105] & $10^{-8}$ \\
\hline
\end{tabular}

With detection limit of $\sim 10^{-3}$, the sensitivity of the magneto photonic crystal optical sensors is comparable with some of the other sensing techniques proposed in literature. In addition our device has a wide sensing range extending from air $(n=1.0)$ to $n=1.6$, making it suitable for variety of applications without stringent temperature stabilization. The sensor sensitivity can be improved by structure modification to enhance the amount of light-matter interaction. The sensitivity enhancement or a reduction in noise level can improve the DL of the device.

\subsection{Sensitive covers}

The use of conducting polymers, such as Polypyrrole, Polyaniline, Polythiophene and their derivatives, as active layers of gas sensors dates back to early 1980s [10]. Some of 
improved features of these materials are that they show high sensitivity, short response time and cost reduction compared to other commercially available sensor materials $[3,10]$. Among the conductive polymers Polypyrrole is one of the most explored as the active component in many sensing techniques for the detection for toxic substances and vapors $[18,19]$. In our study we have integrated PPy with magneto-photonic crystal optical sensors to detect aqueous ammonia and methanol. A PPy thin film deposited on the PC undergoes change in electronic and optical properties when exposed to the analytes. The changes in PC cover environment trigger changes in output polarization rotation, the transduction signal of the MPC sensor.

\subsubsection{Polypyrrole thin films}

Polypyrrole can be synthesized by chemical oxidation of Pyrrole monomer [99]. Vapor phase deposition with Iron (III) chloride hexahydrate $\left(\mathrm{FeCl}_{3} \cdot 6 \mathrm{H}_{2} \mathrm{O}\right)$ as oxidant solution was employed in our tests to obtain Polypyrrole cover layers on the photonic crystal samples. $0.1 \mathrm{M}$ oxidant solution in acetone was deposited on the PC using an optical fiber attached to a micro manipulator. The oxidant deposited sample was then exposed to Pyrrole vapor for 10 seconds where the monomer solution was kept in a $60^{\circ} \mathrm{C}$ water bath. A photonic crystal with a thin layer of black color Polypyrrole is shown in Fig. 4.25 . 


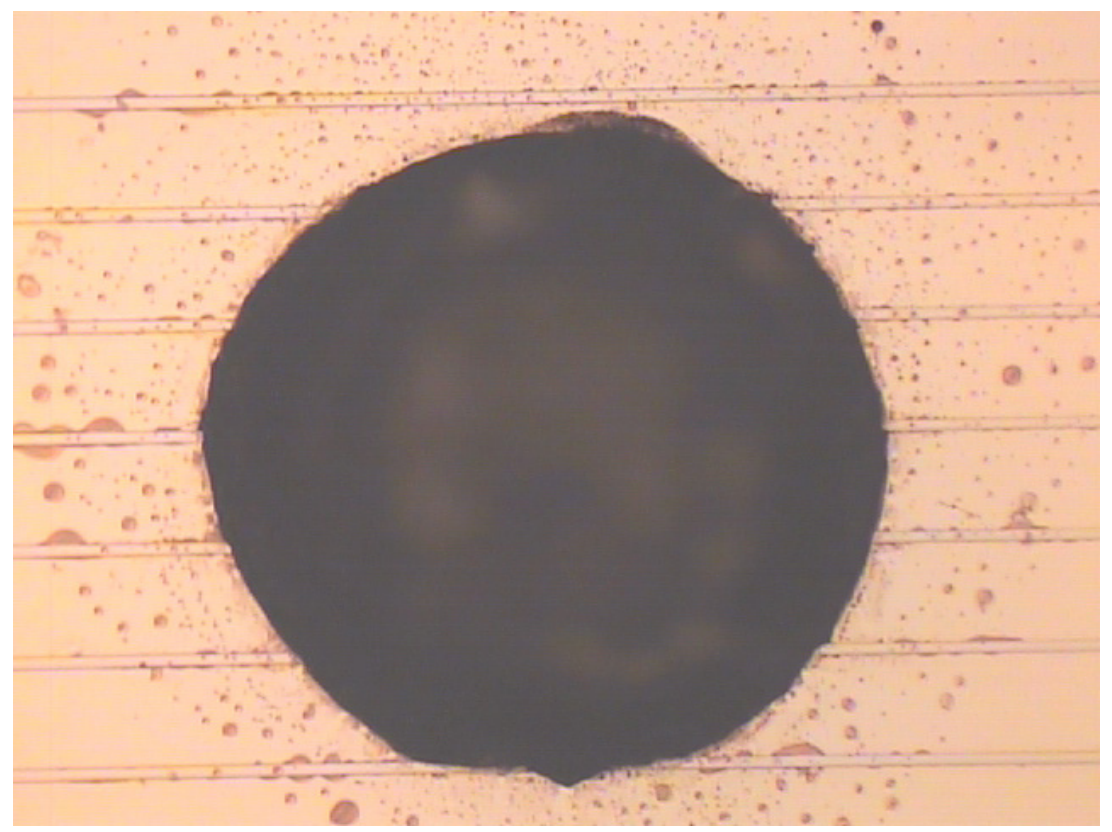

Figure 4.25 Polypyrrole deposited on a photonic crystal sample

\subsubsection{Sensor optical testing}

Transmittance and polarization rotation measurements were carried out on Polypyrrole deposited photonic crystal samples before and after exposure to analyte solutions. Optical transmission was measured for TE input light by end-fire fiber coupling from a 1480-1580nm tunable laser source. PR of the output light was determined by measuring the Stokes vector parameters. All the optical measurements were done in a saturating magnetic field of 3000 applied along the waveguide axis. Polarization rotation measured in one of the test PCs before and after deposition of Polypyrrole is shown in Fig. 4.26. The polarization rotation response for PPy cover is significantly higher especially in the band edge region. 


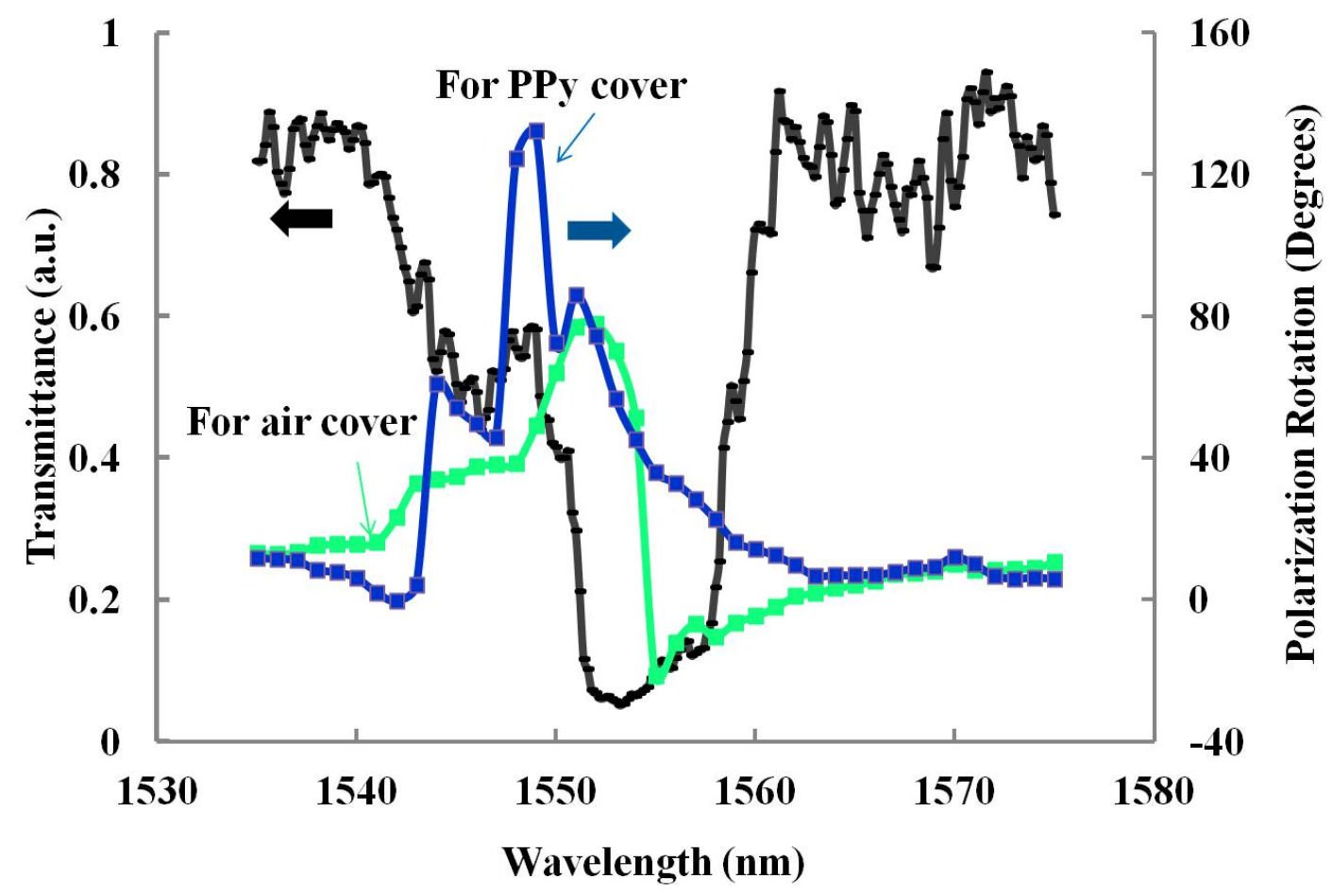

Figure 4.26 Polarization rotation of a test PC measured for air and PPy cover. Transmittance is measured for air cover.

The deposition of PPy reduces the overall transmittance through the waveguide PC, however the shift of the band position is limited to 1-2nm maximum and the coupling strength to the PC remains intact. Figures 4.27 (a) and (b) show the actual transmittance intensity and normalized transmittance for air and PPy cover respectively. 
(a)
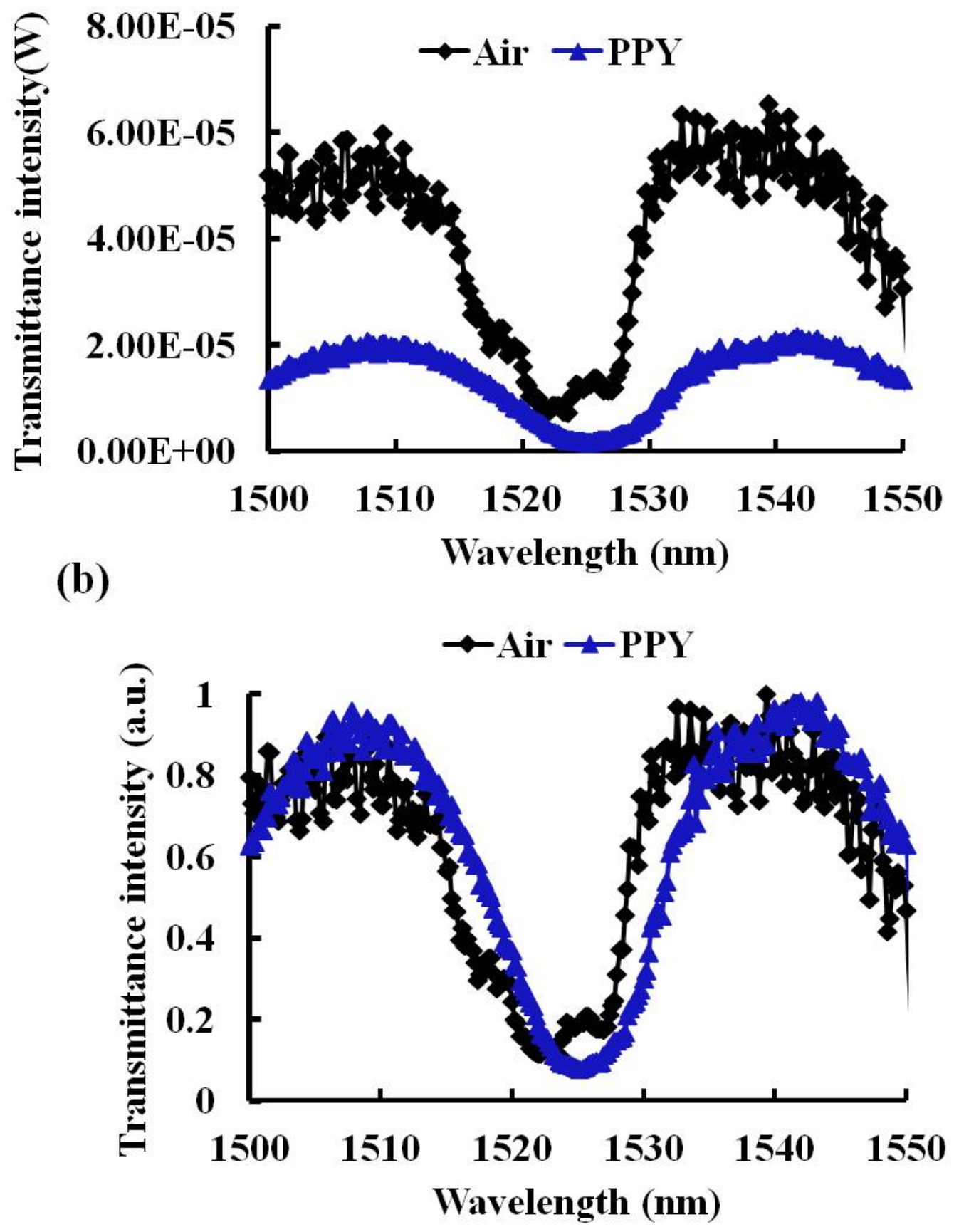

Figure 4.27 (a) Transmittance intensity of a waveguide PC for air and PPy cover. (b) Transmittance intensity normalized with respect to the intensity outside the band gap region for air and PPy cover. 


\subsubsection{Tests with aqueous ammonia and methanol}

Polypyrrole-deposited PC samples were exposed to $29 \%$ an aqueous ammonia solution and laboratory grade methanol. The exposure process consisted of depositing a minute drop $(\sim 10 \mu \mathrm{L})$ of liquid methanol or ammonia on the cover layer and allowing it to dry. A significant change in polarization rotation response was observed upon exposure to both aqueous ammonia and methanol as shown in Figs. 4.28 and 4.29.

PPy thin films are considered to be a good sensing material for $\mathrm{NH}_{3}$ detection $[73,79]$. The decrease in conductivity of PPy on exposure to NH3 was first reported by Kanazawa et al. [105]. PPy undergoes both reversible and irreversible interactions depending on the nature of the $\mathrm{NH}_{3}$ exposure. That is, PPy undergoes irreversible changes when exposed to high concentrations for longer exposure times and reversible changes at low concentrations and short exposure times $[105,106]$. In our tests, the observed polarization rotation changes upon exposure to aq. ammonia and methanol remained constant over time. This was confirmed by repeating the polarization rotation tests after a week from the initial tests. The change of PR to ammonia exposure was consistently $\sim 50^{\circ}$ at the peak of the spectral response, while for methanol, it was $10^{\circ}$ for multiple samples tested. The sensor exhibits a high degree of polarization-rotation selectivity between the two analytes due to the difference in electronic interaction of each analyte with the PPy chains. Both ammonia and methanol are electron donating substances. When they interact with PPy, which is in a doped state by oxidation with $\mathrm{FeCl}_{3} \cdot 6 \mathrm{H}_{2} \mathrm{O}$, it becomes de-doped, causing change in electrical conductivity, hence a change in refractive index. It can be concluded that polarization rotation is highly influenced by the PPy-analyte interaction yielding high sensitivity and selectivity. 
(a)

- PPy cover $=$-PPy cover with aq.ammonia exposed

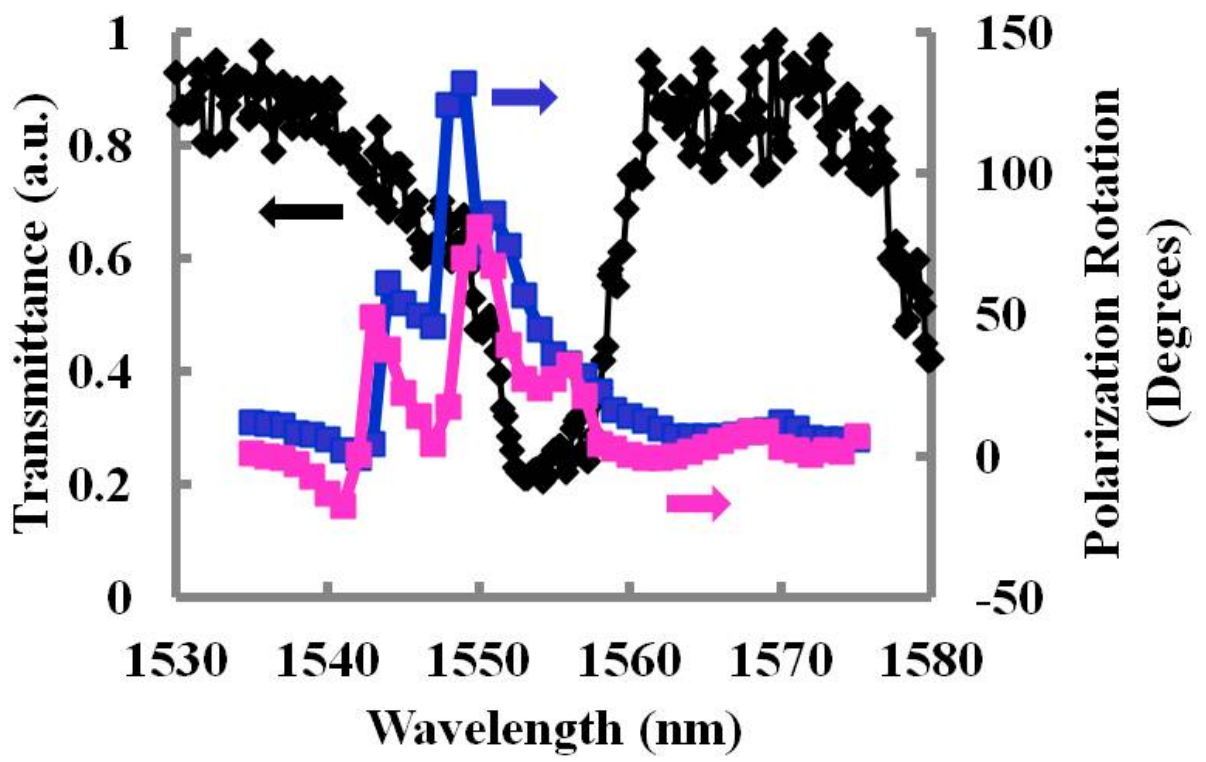

(b)

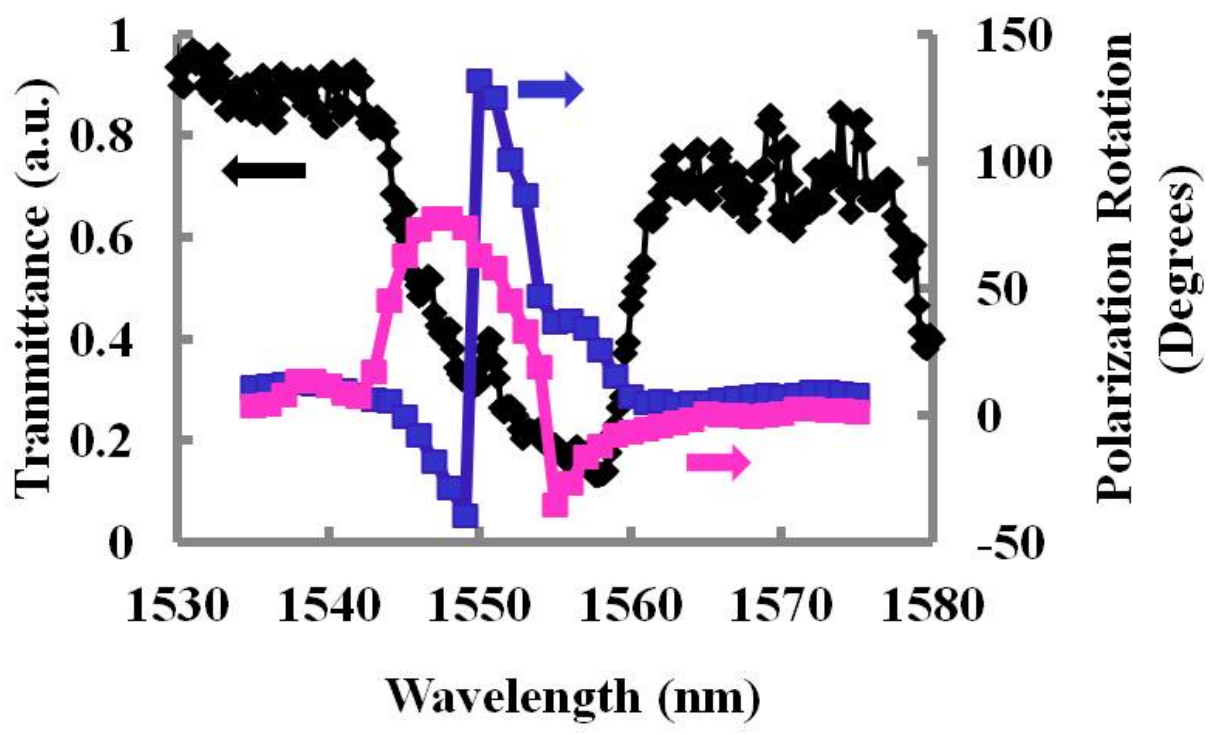

Figure 4.28 (a), (b) Measured transmittance and polarization response of a onedimensional Bragg filter without phase shift step patterned on a $2.7 \mu \mathrm{m}$-thick $\mathrm{Bi}_{0.8} \mathrm{Gd}_{0.2} \mathrm{Lu}_{2.0} \mathrm{Fe}_{5} \mathrm{O}_{12}$ film. The blue and magenta color data points show output polarization rotation of PC with PPy cover and PPy cover exposed to aqueous ammonia. Measurements are taken at magnetic field applied collinear to the waveguide axis. 
(a)

$\rightarrow$ PPy cover $\rightarrow$ Ppy cover with methanol exposed
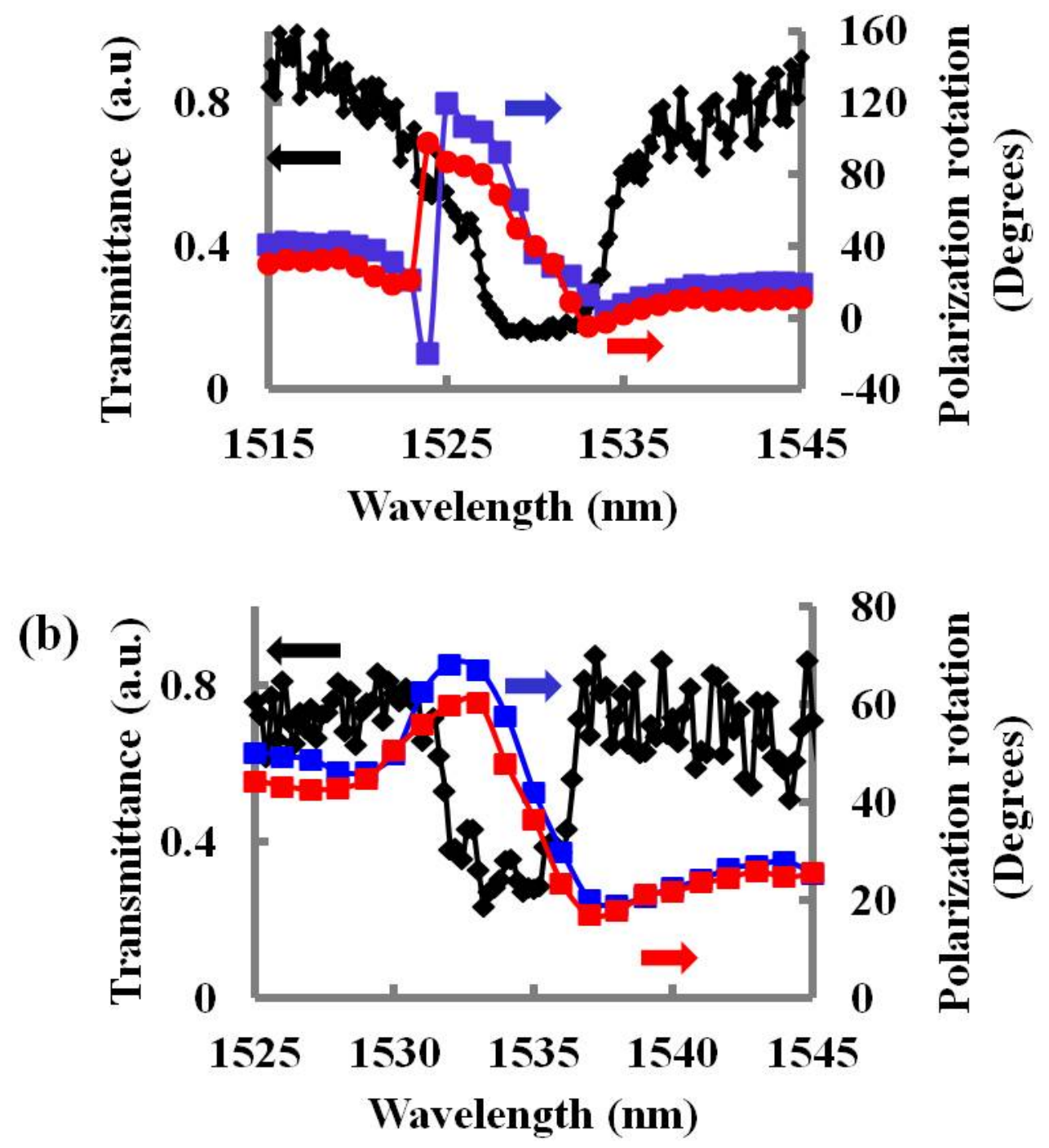

Figure 4.29 (a), (b) Measured transmittance and polarization response of a onedimensional Bragg filter without phase shift step patterned on a $2.7 \mu \mathrm{m}$-thick $\mathrm{Bi}_{0.8} \mathrm{Gd}_{0.2} \mathrm{Lu}_{2.0} \mathrm{Fe}_{5} \mathrm{O}_{12}$ film. The blue and red color data points show output polarization rotation of PC with PPy cover and PPy cover exposed to methanol. Measurements are taken at magnetic field applied collinear to the waveguide axis. 


\subsubsection{Ellipsometric characterization of Polypyrrole}

Ellipsometry is a nondestructive optical technique which uses the changes in the state of polarization of light upon reflection for characterization of surfaces, interfaces and thin films. In this study, ellipsometry was used to measure the refractive index of the Polypyrrole thin films. An interferometric image of a Polypyrrole thin film deposited on silicon substrate is shown in Fig. 4.30.

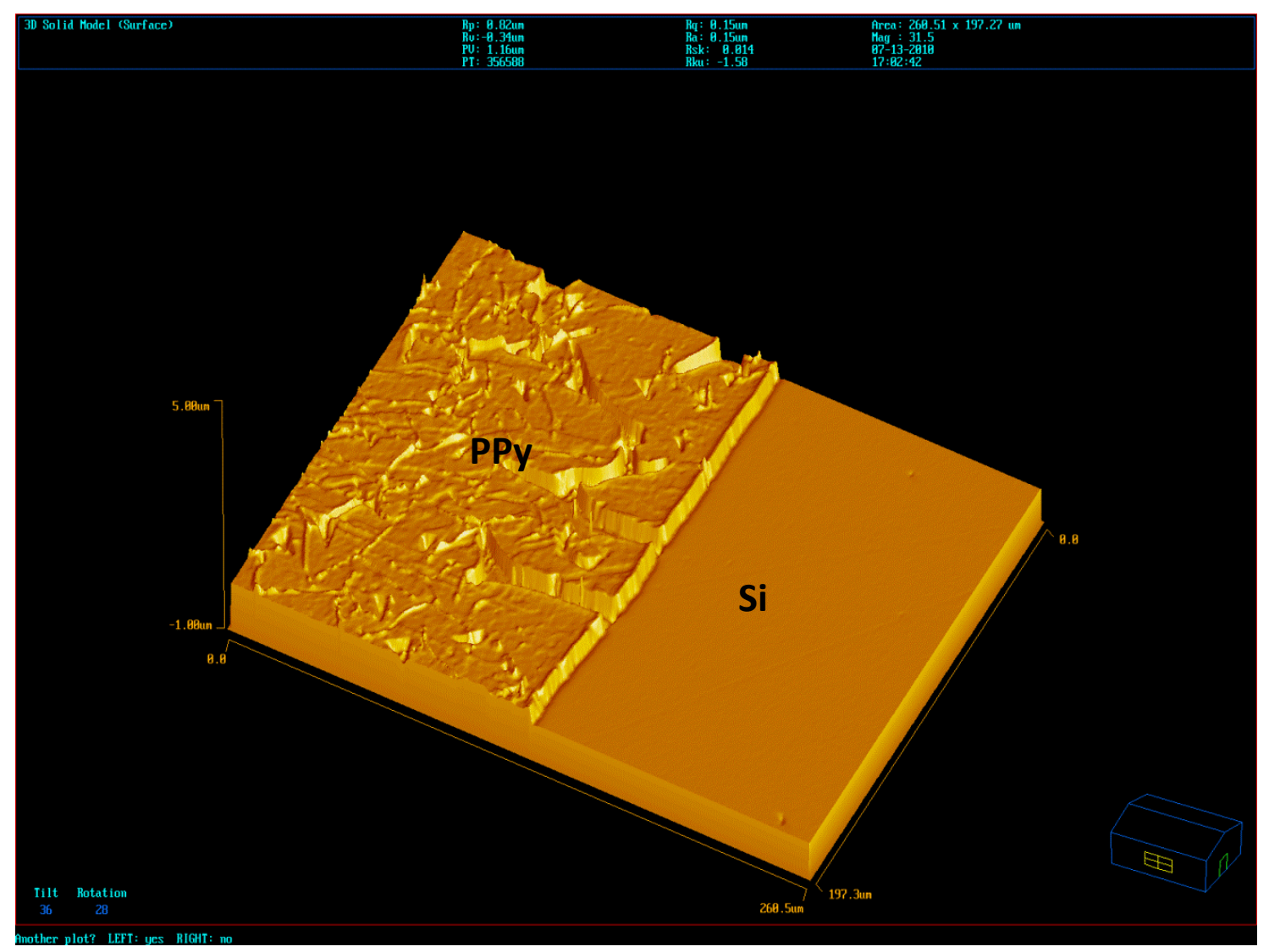

Figure 4.30 An interferometric image of a Polypyrrole (PPy) thin film deposited on a silicon ( $\mathrm{Si}$ ) substrate.

Thin films were deposited by keeping the silicon substrates in a mixture of the oxidant solution $\left(\mathrm{FeCl}_{3} \cdot 6 \mathrm{H}_{2} \mathrm{O}\right)$ and the Pyrrole monomer for 15 minutes. Figure 4.31 shows the $\Psi$ versus wavelength spectra for the experimental and the model fit for the 15 minute deposited film. The recorded refractive index of the thin film is 1.66 at $1550 \mathrm{~nm}$ 
wavelength. Next, the Polypyrrole thin film was exposed to aqueous ammonia. This was achieved by spraying aqueous ammonia from a syringe without removing the film from the ellipsometer set-up. The recorded refractive index of the aqueous ammonia exposed thin film is 1.50 at $1550 \mathrm{~nm}$.

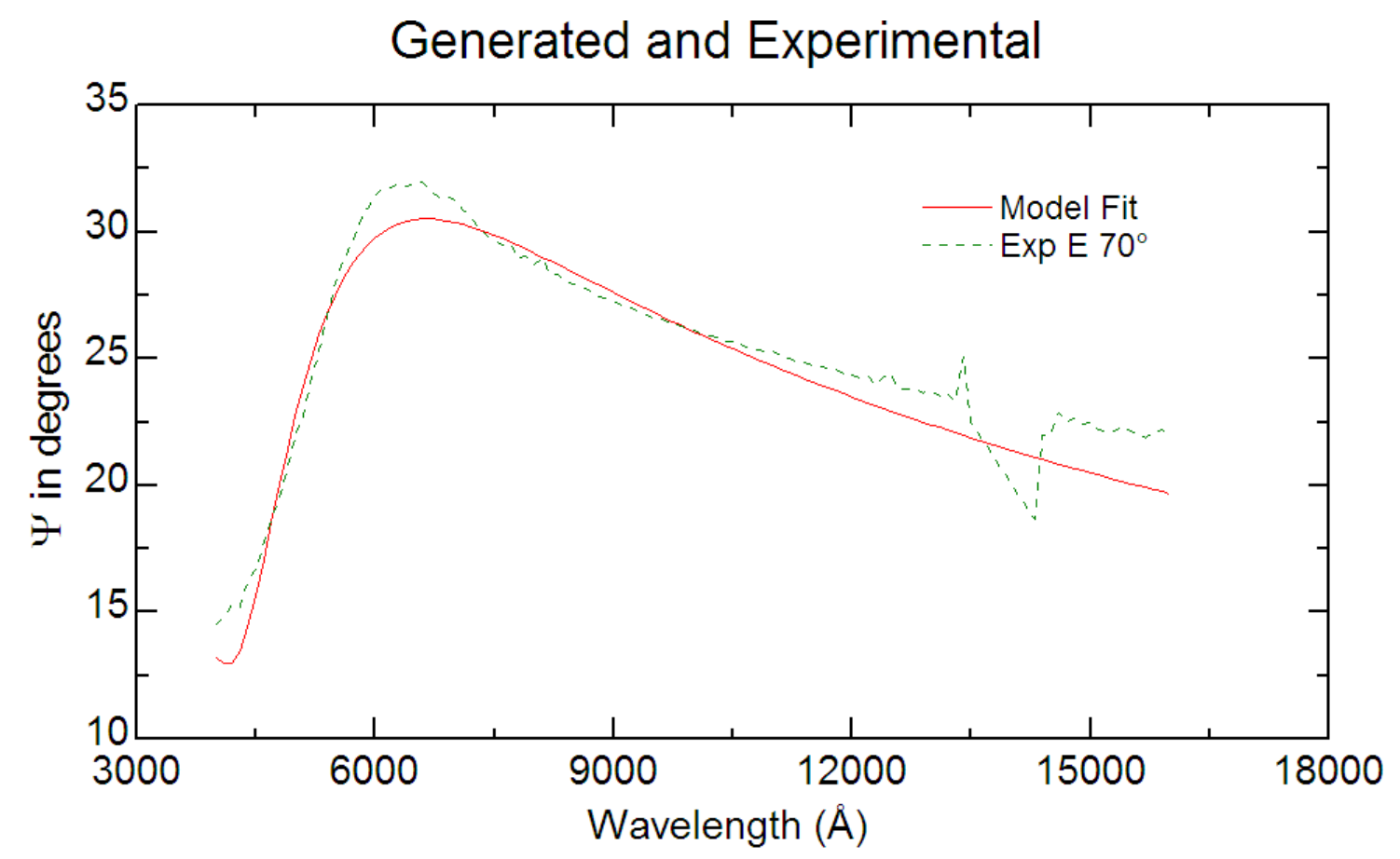

Figure $4.31 \Psi$ versus wavelength spectra for the experimental and the model fit for a Polypyrrole thin film deposited on a silicon substrate using ellipsometry.

The ellipsometric characterization shows a 10\% reduction in refractive index in aqueous ammonia exposed Polypyrrole. This observation agrees well with the polarization rotation response of the optical sensor to aqueous ammonia discussed in Sect. 4.5.3. A decrease in the output polarization rotation in the magneto-photonic crystal sensor represents a decrease in the refractive index. Oxidized Polypyrrole de-dopes under ammonia atmosphere leading to a reduction in the refractive index $[10,105]$. 


\section{SUMMARY AND FUTURE WORK}

\subsection{Summary}

The study developed under this dissertation research introduces a novel optical sensing technique for detection of liquid analytes. It combines photonic crystal technology with magneto-optic polarization rotation to achieve highly sensitive detection of cover index changes in optical waveguides.

We have experimentally and theoretically demonstrated that the one dimensional magneto-photonic crystals fabricated in waveguide geometry yield high polarization rotations even in the absence of a resonant cavity in the structure. Hence it is evident that the polarization rotation enhancement mechanism in these systems is not due to photon trapping in the resonant cavity. Also the photonic crystals which are fabricated in materials with high linear birefringence show comparable higher polarization rotation output. We propose that the polarization rotation enhancement in these systems is due to selective back-reflection of polarization components by the photonic crystal.

The magneto-photonic-crystal-enhanced polarization rotations are found to vary with the nature of the cladding in waveguide configurations. We have used this phenomenon to develop a novel optical sensing technique for chemical analyte detection. Sensor testing was conducted by modifying the photonic crystal cover layer with refractive index liquids of known indices. A wide index range from air to $\mathrm{n}=1.6$ is explored.

The sensing device was further developed for selective analyte detection by employing active sensing layers as photonic crystal covers. We have used a conductive polymer, Polypyrrole as the modified photonic crystal cover layer. The polarization rotation change upon analyte adsorption in sensor layer was used as the transduction signal. A successful detection of ammonia and methanol were demonstrated with high degree of selectivity with Polypyrrole cover layer in the magneto-photonic crystal. 


\subsection{Future work}

The optical sensing technique developed in this work possesses the advantages of high selectivity and low analyte consumption along with room temperature operational capability. However, further research can be done to improve the sensor performance in terms of sensor sensitivity.

The sensitivity and selectivity of sensing the layer can be improved by introducing different dopants and incorporating second component into the conducting polymer. Doping can modify the physical and chemical properties of conducting polymers and influence the sensitivity. Incorporating a second component into the conducting polymer film can adjust the properties of the polymer, such as space between molecules or dipole moments which consequently improve the interactions with analytes [10]. This may also increase the solubility of conducting polymers. C.P. de Melo et.al have reported that some of the polymer blends (PMMA/PPy, PVA/PPy) show distinct level of responses towards polar and apolar analytes [107].

In addition to the improvements to the sensor layer, the overall sensitivity of the device can be improved by increasing the light-matter interaction. The interaction volume can be increased by fabricating photonic crystals with deeper groves. Also it would be interesting to investigate the device performance for a two dimensional air hole array structure. 


\section{REFERENCES}

1. X. Fan, I. M. White, S. I. Shopova, H. Zhu, J. D. Suter, Y. Sun, "Sensitive optical biosensors for unlabeled targets: A review", Analytica Chimica Acta 2008, 620, 8-26.

2. Colette McDonagh, Conor S. Burke, and Brian D. MacCraith, Optical Chemical Sensors, Chem. Rev. 2008, 108, 400-422.

3. Mahmoud El-Sherif, Lalitkumar Bansal, Jianming Yuan, "Fiber Optic Sensors for Detection of Toxic and Biological Threats", Sensors 2007, 7, 3100-3118.

4. Paul V Lambeck, "Integrated optical sensors for the chemical domain", Meas. Sci. Technol. 17 (2006) R93-R116.

5. E. Chow, A. Grot, L. W. Mirkarimi, M. Sigalas, and G. Girolami, "Ultracompact biochemical sensor built with two-dimensional photonic crystal microcavity", Opt. Lett. 29, 1093 (2004).

6. Xing-Jiu Huang and Yang-Kyu Choi, "Chemical sensors based on nanostructured materials", Sensors and Actuators B 122 (2007) 659-671.

7. Lars Rindorf, Jesper B. Jensen, Martin Dufva, Lars Hagsholm Pedersen, Poul Erik Høiby, Ole Bang, "Photonic crystal fiber long-period gratings for biochemical sensing”, Optics Express 14, 8224(2006).

8. Nina Skivesen, Amélie Têtu, and Martin Kristensen, Jørgen Kjems, Lars H. Frandsen and Peter I. Borel, "Photonic-crystal waveguide biosensor", Optics Express 15, 3169(2007).

9. Jing $\mathrm{Wu}$, Daniel Day, Min Gu, "A microfluidic refractive index sensor based on an integrated three-dimensional photonic crystal”, Appl. Phys. Lett. 92, 071108 (2008).

10. Hua Bai and Gaoquan Shi, "Gas Sensors Based on Conducting Polymers", Sensors 2007, 7, 267-307.

11. Miguel Levy and Rong Li, "Polarization rotation enhancement and scattering mechanisms in waveguide magnetophotonic crystals", Appl. Phys. Lett. 89, $121113(2006)$. 
12. Miguel Levy and Amir A. Jalali, "Band structure and Bloch states in birefringent 1D magnetophotonic crystals: An analytical approach," Journal of the Optical Society of America B 24, 1603-1609, July 2007.

13. A.A. Jalali and M. Levy, 'Local normal mode coupling and energy band splitting in elliptically birefringent 1D magneto photonic crystals', J. Opt. Soc. Am. B 25, 119 (2008).

14. Miguel Levy, Amir A. Jalali, Ziyou Zhou and Neluka Dissanayake, "Bandgap formation and selective suppression of Bloch states in birefringent gyrotropic Bragg waveguides," Optics Express 16, 13421-13430, Aug 2008.

15. Amir A Jalali, Miguel Levy, Ziyou Zhou and Neluka Dissanayake, "Polarization rotation enhancement and gyrotropic photonic bandgaps in birefringent magneto-photonic crystals", Photonic Fiber and Crystal Devices: Advances in Materials and Innovations in Device Applications II, Proceedings of the SPIE, Volume 7056, 70560U (2008).

16. Neluka Dissanayake, Miguel Levy, A. A. Jalali, V.J. Fratello, "Gyrotropic Band Gap Optical Sensors," Applied Physics Letters 96, Issue 18, May 2010.

17. Neluka Dissanayake, Miguel Levy, A. Chakravarty, P. A. Heiden, N. Chen, and V. J. Fratello, " Magneto-photonic crystal optical sensors with sensitive covers," Applied Physics Letters 99, Issue 09, Sep 2011.

18. Qaisar Ameer and Samuel B. Adeloju, 'Polypyrrole-based electronic noses for environmental and industrial analysis', Sensors and Actuators B 106, 541552(2005).

19. T V Vernitskaya and O N Efimov, 'Polypyrrole: a conducting polymer; its synthesis, properties and applications', Russian Chemical Reviews 66 (5) 443 457 (1997).

20. H. Nishihara, M. Haruna, T. Suhara, "Optical Integrated Circuits", McGrawHill, New York,(1987).

21. R. Wolfe, V. J. Fratello, and M. McGlashanPowell, "Elimination of birefringence in garnet films for magnetooptic waveguide devices", Appl. Phys. Lett. 51, 1221 (1987).

22. R. Wolfe, V. J. Fratello, and M. McGlashan-Powell, "Thin film garnet materials with zero linear birefringence for magnetooptic waveguide devices (invited)", J. Appl. Phys. 63(8), 3099 (1988). 
23. Koji Ando, Norio Takeda, Naoki Koshizuka, Takashi Okuda, "Annealing effects on growthinduced optical birefringence in liquidphase epitaxialgrown Bisubstituted iron garnet films", J. Appl. Phys. 57, 1277 (1985).

24. "Magnetism I - Fundamentals", edited by E. du Tremolet de Lacheisserie, D. Gignoux, and M.Schlenker, Kluwer Academic Pub. (2002).

25. G. F. Dionne, G.A. Allen, P. R. Haddad, C. A. Ross and B. Lax, Lincoln Lab. VOLUME 15, NUMBER 2, (2005).

26. Nicola Spaldin, "Magnetic Materials, Fundamentals and Device Applications", Cambridge University Press.

27. M. Levy, "Normal modes and birefringent magnetophotonic crystals", J. Appl. Phys. 99, 073104(2006)

28. R. Li, "Fabrication and characterization of planar magneto-photonic crystals", $\mathrm{PhD}$. Thesis (2006).

29. S. Kahl and A. M. Grishin, "Enhanced Faraday rotation in all-garnet magnetooptical photonic crystal," Appl. Phys. Lett. 84, 1438-1440 (2004).

30. Min Huanga and Zhi-Chun Xub, "Liquid phase epitaxy growth of bismuthsubstituted yttrium iron garnet thin films for magneto-optical applications", Thin Solid Films 450 324-328 (2004).

31. S. Kahl, "Bismuth iron garnet films for magneto-optical photonic crystals", Doctoral Dissertation (2004).

32. T. Aichele, A. Lorenz1, R. Hergt, P. Görnert, "Garnet layers prepared by liquid phase epitaxy for microwaveand magneto-optical applications - a review", Cryst. Res. Technol. 38, No. 7-8, 575 - 587 (2003).

33. Horst Dotsch, Norbert Bahlmann, Oleksandr Zhuromskyy, Manfred Hammer,Ludger Wilkens, Reinald Gerhardt, Peter Hertel, Anatoly F. Popkov, "Applications of magneto-optical waveguides in integrated optics: review", J. Opt. Soc. Am. B, 22, (2005).

34. Pete Vukusic and J. Roy Sambles, "Photonic structures in biology", Nature, 424, (2003).

35. Eli Yablonovitch, Inhibited Spontaneous Emission in Solid-State Physics and Electronics. Phys. Rev. Lett.; 58: 2059(1987). 
36. John S.. Strong localization of photons in certain disordered dielectric superlattices. Phys. Rev. Lett.; 58(23):2486-2489(1987).

37. J.D. Joannopoulos, R.D. Meade, J.N.Winn, Photonic Crystals: Molding the Flow of Light, Princeton University Press, Princeton, NJ, (1995).

38. S. G. Johnson and J. D. Joannopoulos, "Introduction to Photonic Crystals: Bloch's Theorem, Band Diagrams, and Gaps (But No Defects)" (2003).

39. Information of photonic crystals, http://ab-initio.mit.edu/photons/.

40. Ho K.M., Chan C.T., Soukoulis C.M.. "Existence of a photonic gap in periodic dielectric structures", Phys. Rev. Lett.; 65: 3152(1990).

41. U. Grüning, V. Lehmann, S. Ottow, and K. Busch. Macroporous silicon with a complete two-dimensional photonic band gap centered at $5 \mu \mathrm{m}$. Appl. Phys. Lett.; 68: 747(1996).

42. R H Lipson and $\mathrm{C} \mathrm{Lu}$, "Photonic crystals: a unique partnership between light and matter", Eur. J. Phys. 30, S33-S48 (2009).

43. G. Feiertag, W. Ehrfeld, H. Freimuth, H. Kolle, H. Lehr, and M. Schmidt. Fabrication of photonic crystals by deep X-ray lithography. Appl. Phys. Lett.; 71: 1441(1997).

44. Albert Birner, Ralf B. Wehrspohn, Ulrich M. Gösele, and Kurt Busch.SiliconBased Photonic Crystals. Adv. Mater.; 13: 377(2001).

45. E. Özbay, A. Abeyta, G. Tuttle, M. Tringides, R. Biswas, C. T. Chan, C. M. Soukoulis, and K. M. Ho. Measurement of a three-dimensional photonic band gap in a crystal structure made of dielectric rods. Phys. Rev.; B 50: 19451948(1994).

46. Johnson S G, Villeneuve P R, Fan S and Joannopoulos J D "Linear waveguides in photonic-crystal slabs" Phys. Rev. B 628212 (2000).

47. Russell P, "Photonic crystal fibers", Science, 299, 358 (2003).

48. Dowling J P, Scalora M, Bloemer M J and Bowden C M The photonic band edge laser: a new approach to gain enhancement J. Appl. Phys. 751896 (1994). 
49. Kosaka H, Kawashima T, Tomita A, Notomi M, Tamamura T, Sato T and Kawakami S “ phenomena in photonic crystals", Phys. Rev. B 58 R10096 (1998)

50. Park H-G, Kim S-H, Kwon S-H, Ju Y-G, Yang J-K, Baek J-H, Kim S-B and Lee Y-H "Electrically driven single-cell photonic crystal laser", Science 3051444 (2004).

51. Noda S, Fujita $\mathrm{M}$ and Asano $\mathrm{T}$ "Spontaneous-emission control by photonic crystals and nanocavities", Nat.Photonics 1 449, (2007).

52. M Inoue, R Fujikawa, A Baryshev1, A Khanikaev, P B Lim, H Uchida, O Aktsipetrov, A Fedyanin, T Murzina and A Granovsky, "Magnetophotonic crystals", J. Phys. D: Appl. Phys. 39 R151-R161, (2006).

53. M. Levy, X. Huang, R. Li, H. C. Yang, H. Bakhru, "Magneto-optic photonic crystals as optical waveguide structures," SPIE Proceedings 5515, 49 ${ }^{\text {th }}$ Annual Meeting, Symposium on Nano-engineering: Fabrication, Properties, Optics and Devices, August 2004.

54. M. Levy, H. C. Yang, M. J. Steel, and J. Fujita, "Flat Top Response in OneDimensional Magnetic Photonic Band Gap Structures with Faraday Rotation Enhancement," IEEE J. Lightwave Technol. 19, 1964-1969 (2001).

55. M. Inoue, K. Isamoto, T. Yamamoto and T. Fujii, J. Appl. Phys. 79(3), 1611(1996).

56. M. Inoue and T. Fujii, "A Theoretical Analysis of Magneto-Optical Faraday Effect of YIG Films with Random Multilayer Structures,” J. Appl. Phys., 81, 5659-5661 (1997).

57. M. Inoue, K. I. Arai, T. Fujii, and M. Abe, "Magneto-Optical Properties of OneDimensional Photonic Crystals Composed of Magnetic and Dielectric Layers," J. Appl. Phys. 83, 6768-6770 (1998).

58. M. Inoue, K. Arai, T. Fujii and M. Abe, "One-dimensional magnetophotonic crystals," J. Appl. Phys., 85, 5768-70 (1999).

59. E. Takeda, N. Todoroki, Y. Kitamoto, M. Abe, M. Inoue, T. Fujii and K. Arai, J. Appl. Phys. 87, 6782 (2000). 
60. M. Levy, "The On-Chip Integration of Magneto-Optic Waveguide Isolators," invited paper IEEE J. of Selected Topics in Quantum Electronics 8, No. 6, 1300 - 1306 (2002).

61. M. J. Steel, M. Levy, and R. M. Osgood Jr., "High Transmission Enhanced Faraday Rotation in One-dimensional Photonic Crystals with Defects," IEEE Photonics Technol. Lett. 12, 1171-1173 (2000).

62. M. J. Steel, M. Levy, and R. M. Osgood, Jr., "Photonic bandgaps with defects and the enhancement of Faraday rotation," J. Lightwave Technol. 18, 1297-1308 (2000).

63. Xiaoyue Huang, "Dimensional effects on the magnetic domains in planar magnetophotonic crystal waveguide", PhD dissertation(2007).

64. Zhuoyuan $\mathrm{Wu}$, "Planar magneto-photonic and gradient-photonic structures: crystals and metamaterials" PhD dissertation (2010).

65. Rong Li and Miguel Levy, "Bragg Grating Magnetic Photonic Crystal Waveguides,” Appl. Phys. Lett. 86, No. 25, 251102, 2005.

66. Rong Li and Miguel Levy, "Erratum: Bragg Grating Magnetic Photonic Crystal Waveguides," [Appl. Phys. Lett. 86, 251102, 2005], Appl. Phys. Lett. 87, 269901, 2005.

67. M. Levy, R. Li, A. A. Jalali and X. Huang "Band edge effects and normal mode propagation in waveguide magnetophotonic crystals," J. of the Magnetics Society of Japan 30, No6-2, 561-566, November 2006.

68. M. Levy, X. Huang, R. Li, H.C. Yang and H. Bakhru, Proc. SPIE Int. Soc. Opt. Eng. 5515, 30 (2004).

69. X. Huang, R. Li, H.C. Yang, M. Levy, "Multimodal and birefringence effects in magnetic photonic crystals," J. Magn. Mag. Materials 300, No.1, 112-116, (2006).

70. Z. Wu, M. Levy, V. J. Fratello, and A. M. Merzlikin, Appl. Phys.Lett. 96, 051125 (2010).

71. X. Huang, R. Li, H.C. Yang, M. Levy, "Multimodal and birefringence effects in magnetic photonic crystals," J. Magn. Mag. Materials, 300, 112-116, May 2006 
72. Ashim Chakravarty, Miguel Levy, Amir A. Jalali, and Zhuoyuan Wu, "Elliptical normal modes and stop band reconfiguration in multimode birefringent onedimensional magnetophotonic crystals", Physical review B 84, 094202 (2011).

73. Q. Ameer, S. B. Adeloju, "Polypyrrole-based electronic noses for environmental and industrial analysis" Sensors and Actuators B 106 541-552 (2005).

74. Y. Nagase, K. Wakabayashi, T. Imanaka, "Effect of doping anions in Polypyrrole gas sensors", Sens. Actuators B 13-14 (1993) 596.

75. Reza Ansari, "Polypyrrole Conducting Electroactive Polymers: Synthesis and Stability Studies", E-Journal of Chemistry Vol. 3, No.13, pp 186-201, October 2006.

76. $\mathrm{T}$ V Vernitskaya, O N Efimov, "Polypyrrole: a conducting polymer; its synthesis, properties and Applications", Russian Chemical Reviews 66 (5) $443 \pm$ 457 (1997).

77. S. Barnossa, H. Shanaka, C.C. Bof Bufonb, T. Heinzela, "Piezoresistance in chemically synthesized polypyrrole thin films", Sensors and Actuators A 154, 79-84 (2009).

78. Lalitkumar Bansal and Mahmoud El-Sherif, "Intrinsic Optical-Fiber Sensor for Nerve Agent Sensing”, IEEE Snsors journal, Vol. 5, No. 4, August 2005.

79. H. J. Kharat, K. P. Kakde, P. A. Savale, K. Datta, P. Ghosh and M. D. Shirsat, "Synthesis of polypyrrole films for the development of ammonia sensor", Polym. Adv. Technol. 18: 397-402(2007).

80. L. B. Freund and S. Suresh, Thin Film Materials, stress, defect formation and surface evolution, Cambridge University press.

81. T. Aichele, A. Lorenz, R. Hergt, P. Görnert, "Garnet layers prepared by liquid phase epitaxy for microwave and magneto-optical applications - a review", Cryst. Res. Technol. 38, No. 7-8, $575-587$ (2003)

82. I. Nistor, C. Holthaus, I. D. Mayergoyz, C. Krafft, "Development of liquid phase epitaxy-grown „Bi, Gd, Lu...-substituted thin-film iron garnets”, Journal of Applied Physics 99, 08M702 (2006) 
83. Vincent J. Fratello, Irina Mnushkina, Steven J. Licht and Robert R. Abbott, "Growth and Characterization of Magnetooptic Garnet Films with Planar Uniaxial Anisotropy”, Mater. Res. Soc. Symp. Proc. Vol. 834 (2005)

84. H. C. Yang, "RF-sputter fabrication of magnetic garnet thin films and simulation modeling for 1-D magnetic photonic crystal waveguide devices", $\mathrm{PhD}$. thesis, (2005).

85. RSoft, Inc. Ossining, NY 10562. (http://www.rsoftdesign.com).

86. Yong Kwan Kim, Aaron J. Danner, James J. Raftery, Jr., and Kent D. Choquette, "Focused Ion Beam Nanopatterning for Optoelectronic Device Fabrication”, IEEE Journal of selected topics in Quantum Electronics, 11 (2005).

87. Ampere A. Tseng, "Recent Developments in Nanofabrication Using Focused Ion Beams", Small, 1, No. 10, 924 - 939 (2005).

88. C.A.Volkert and A.M. Minor, "Focused Ion Beam Microscopy and Micromachining", MRS Bulletin, 32 (2007)

89. J.C. Nabity, "Nano Pattern Generation System, User's manual for NPGS v8 \& v9".

90. R. Chipman, in Handbook of Optics, M. Bass, E. W. Van Stryland, D. R. Williams, and W. L. Wolfe, eds. (Optical Society of America, Washington, D.C., 1994), Vol. II, pp. 22.1-22.22.

91. Leonardo Giudicotti and Matteo Brombin, "Data analysis for a rotating quarterwave, far-infrared Stokes polarimeter”, Applied Optics , Vol. 46, No. 14 (2007).

92. Rasheed M. A. Azzam, in Handbook of Optics, M. Bass, E. W. Van Stryland, D. R. Williams, and W. L. Wolfe, eds. (Optical Society of America, Washington, D.C., 1994), Vol. I, Chapter 27.

93. J. A. Woollam, B. Johs, C. Herzinger, J. Hilfiker, R. Synowicki, and C. Bungay, "Overview of Variable Angle Spectroscopic Ellipsometry (VASE), Part I: Basic Theory and Typical Applications”, SPIE Proceedings, CR72, (1999) 3-28.

94. http://www.jawoollam.com/tutorial.

95. K. Lorenz, H. Dotsch, W. Tolksdorf, and P. Willicha, "Influence of growth conditions and annealing parameters on the near infrared optical absorption of epitaxial magnetic garnet films", J. Appl. Phys. 71, 436 (1992). 
96. Cargille Laboratories, Cedar Grove, New Jersey 07009.

97. http://www.metricon.com/basic.html

98. Ian M. White and Xudong Fan, "On the performance quantification of resonant refractive index sensors”, OPTICS EXPRESS Vol. 16, No. 2, January 2008.

99. Bansal, L. Development of a fiber optic chemical sensor for detection of toxic vapor. PhD Thesis, Dec. 2004, Advisor: Dr. Mahmoud A. El-Sherif.

100. Pedro S. Nunes, Niels Asger Mortensen, Jörg P. Kutter and Klaus B. Mogensen,

"Refractive Index Sensor Based on a 1D Photonic Crystal in a Microfluidic Channel", Sensors, 10, 2348-2358 (2010).

101. E. Chow, A. Grot, L.W. Mirkarimi, M. Sigalas, G. Girolami, Opt. Lett. 29 (2004) 1093.

102. M.R. Lee, P.M. Fauchet, Opt. Express 15 (2007) 4530.

103. L. Rindorf, and O. Bang, "Highly sensitive refractometer with a photoniccrystal-fiber long-period grating," Opt. Lett. 33(6), 563-565 (2008).

104. F. Xu, P. Horak, G. Brambilla, Opt. Express 15 (2007) 9385.

105. K.K. Kanazawa, A.F. Diaz, R.H. Geiss, W.D. Gill, J.F. Kwak, J.A. Logan, J.F. Rabolt, G.B. Street, Organic metals: polypyrrole, a stable synthetic metallic polymer, J. Chem. Soc. Chem. Commun. ,854-855 (1979).

106. G.Gustafsson, I Lundstrom, "The effect of ammonia on the physical properties of Polypyrrole", Synthetic Metals, 21, 203-208 (1987).

107. C.P. de Meloa, B.B. Neto, E.G. de Lima, L.F.B. de Lira, J.E.G. de Souza, "Use of conducting polypyrrole blends as gas sensors", Sensors and Actuators B 109, 348-354 (2005). 


\section{PUBLICATIONS/PATENT APPLICATIONS}

1. Neluka Dissanayake, Miguel Levy, A. Chakravarty, P. A. Heiden, N. Chen, and V. J. Fratello, " Magneto-photonic crystal optical sensors with sensitive covers," Appl. Phys. Lett. 99, 091112, Sept 2011.

2. Neluka Dissanayake, Miguel Levy, A. A. Jalali, V.J. Fratello, "Gyrotropic band gap optical sensors," Appl. Phys. Lett. 96, 181105, May 2010.

3. Miguel Levy, Amir A. Jalali, Ziyou Zhou and Neluka Dissanayake, "Bandgap formation and selective suppression of Bloch states in birefringent gyrotropic Bragg waveguides," Optics Express 16, 13421-13430, Aug 2008.

4. Amir A Jalali, Miguel Levy, Ziyou Zhou and Neluka Dissanayake, "Polarization rotation enhancement and gyrotropic photonic bandgaps in birefringent magnetophotonic crystals", Photonic Fiber and Crystal Devices: Advances in Materials and Innovations in Device Applications II, Proceedings of the SPIE, Volume 7056, $70560 \mathrm{U}(2008)$.

\section{Patent Application:}

Miguel Levy, Amir A Jalali, Neluka Dissanayake, "Degenerate Band Gaps In Magneto-Photonic Media and Magneto-Photonic Crystal Biochemical Sensors", United States Provisional Patent filed, Application No. 61/340,642. 


\section{APPENDIX A}

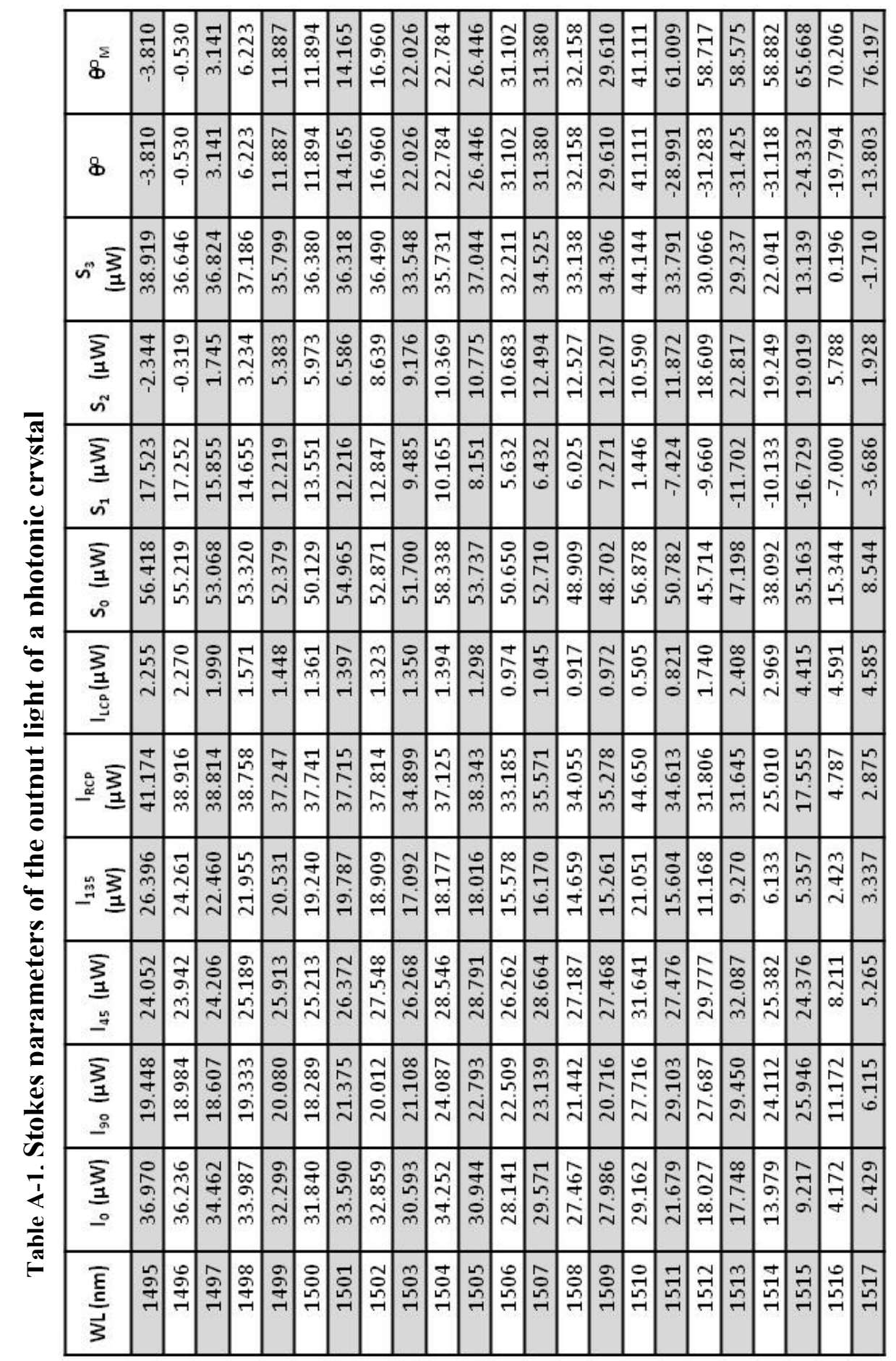




\section{APPENDIX B}

Permissions from Authors/Companies to use their figures for this dissertation

\section{Figure 1.1}

pubscopyright < copyright@osa.org> Mon, Jul 23, 2012 at 1:50 PM

To: Neluka Dissanayake <nkdissan@mtu.edu>

Dear Neluka,

Thank you for contacting The Optical Society.

OSA considers your requested use of its copyrighted material to be Fair Use under United States Copyright Law. It is requested that a complete citation of the original material be included in any publication.

Let me know if you have any questions.

Best wishes,

Hannah

Hannah Bembia

July 23, 2012

Authorized Agent, The Optical Society

Note: To comply with the copyright requirements of the OSA, the articles subjected to this request are fully cited and listed in the Reference list as [5] and [8]. 


\section{Figure 2.9}

ZhuoyuanWu <zhuoyuan.wu@seagate.com>

Wed, Jul 25, 2012 at 1:39 AM

To: Neluka Dissanayake<nkdissan@mtu.edu>

Hello Neluka,

You have my permission to use the figure.

Thanks,

zhuoyuan wu 